Draft Version April 12, 2017

Preprint typeset using LATEX style AASTeX6 v. 1.0

\title{
GAMMA RAY BURST AFTERGLOW AND PROMPT-AFTERGLOW RELATIONS: AN OVERVIEW
}

\author{
Dainotti M. G. ${ }^{1,2,3}$, Del Vecchio R. ${ }^{3}$ \\ ${ }^{1}$ Physics Department, Stanford University, Via Pueblo Mall 382, Stanford, CA, USA, E-mail: mdainott@stanford.edu \\ ${ }^{2}$ INAF-Istituto di Astrofisica Spaziale e Fisica cosmica, Via Gobetti 101, 40129, Bologna, Italy \\ ${ }^{3}$ Astronomical Observatory, Jagiellonian University, ul. Orla 171, 30-244 Kraków, Poland E-mails: delvecchioroberta@hotmail.it, mariagio- \\ vannadainotti@yahoo.it
}

\begin{abstract}
The mechanism responsible for the afterglow emission of Gamma Ray Bursts (GRBs) and its connection to the prompt $\gamma$-ray emission is still a debated issue. Relations between intrinsic properties of the prompt or afterglow emission can help to discriminate between plausible theoretical models of GRB production. Here we present an overview of the afterglow and prompt-afterglow two parameter relations, their physical interpretations, their use as redshift estimators and as possible cosmological tools. A similar task has already been correctly achieved for Supernovae (SNe) Ia by using the peak magnitude-stretch relation, known in the literature as the Phillips relation (Phillips 1993). The challenge today is to make GRBs, which are amongst the farthest objects ever observed, standardizable candles as the SNe Ia through well established and robust relations. Thus, the study of relations amongst the observable and physical properties of GRBs is highly relevant together with selection biases in their physical quantities.

Therefore, we describe the state of the art of the existing GRB relations, their possible and debated interpretations in view of the current theoretical models and how relations are corrected for selection biases. We conclude that only after an appropriate evaluation and correction for selection effects can GRB relations be used to discriminate among the theoretical models responsible for the prompt and afterglow emission and to estimate cosmological parameters.
\end{abstract}

Keywords: gamma rays bursts, accretion model, LT relation. 


\section{Contents}

1. Introduction

3. The Afterglow Relations

3.1. The Dainotti relation $\left(L_{X}\left(T_{a}\right)-T_{X, a}^{*}\right)$

3.1.1. Physical interpretation of the Dainotti relation $\left(L_{X}\left(T_{a}\right)-T_{X, a}^{*}\right)$

3.2. The unified $L_{X}\left(T_{a}\right)-T_{X, a}^{*}$ and $L_{O, a}-T_{O, a}^{*}$ relations

3.2.1. Physical interpretation of the unified $L_{X}\left(T_{a}\right)-T_{X, a}^{*}$ and $L_{O, a}-T_{O, a}^{*}$ relations

3.3. The $L_{O, 200 \mathrm{~s}}-\alpha_{O,>200 \mathrm{~s}}$ relation and its physical interpretation

4. The Prompt-Afterglow Relations

18

18

20

4.2. The $L_{X \text {, afterglow }}-E_{\gamma, \text { prompt }}$ relation and its physical interpretation

4.3. The $L_{X, a}-L_{O, a}$ relation and its physical interpretation

25

4.4. The $L_{X}\left(T_{a}\right)-L_{\gamma, \text { iso }}$ relation

4.5. The $L_{X, \text { peak }}-L_{X}\left(T_{a}\right)$ relation

4.5.1. Physical interpretation of the $L_{X}\left(T_{a}\right)-L_{\gamma, \text { iso }}$ and the $L_{X \text {,peak }}-L_{X}\left(T_{a}\right)$ relations

4.6. The $L_{O, p e a k}^{F}-T_{O, p e a k}^{* F}$ relation and its physical interpretation

5. Selection Effects

5.1. Redshift induced relations

5.2. Redshift induced relations through Efron and Petrosian method

5.2.1. Luminosity evolution

5.2.2. Time Evolution

5.3. Evaluation of the intrinsic slope

5.4. Selection effects for the optical and X-ray luminosities

6. Redshift Estimator

7. Cosmology

7.1. The problem of the calibration

7.2. Applications of GRB afterglow relations 


\section{INTRODUCTION}

GRBs, amongst the farthest and the most powerful objects ever observed in the Universe, are still a mystery after 50 years from their discovery time by the Vela Satellites (Klebesadel et al. 1973). Phenomenologically, GRBs are traditionally classified in short SGRBs $\left(T_{90}<2 \mathrm{~s}\right)$ and long LGRBs $\left(T_{90}>2 \mathrm{~s}\right)$ (Mazets et al. 1981; Kouveliotou et al. 1993), depending on their duration, where $T_{90}$ is the time in which the $90 \%$ (between $5 \%$ and $95 \%$ ) of radiation is emitted in the prompt emission. However, Norris and Bonnell (2006) discovered the existence of an intermediate class (IC), or SGRBs with Extended Emission (SGRBsEE), that shows mixed properties between SGRBs and LGRBs. Another relevant classification related to the spectral features distinguishing normal GRBs from X-ray Flashes (XRFs) appears. The XRFs (Heise et al. 2001; Kippen et al. 2001) are extra-galactic transient X-ray sources with spatial distribution, spectral and temporal characteristics similar to LGRBs. The remarkable property that distinguishes $\mathrm{XRFs}$ from GRBs is that their $\nu F_{\nu}$ prompt emission spectrum peaks at energies typically one order of magnitude lower than the observed peak energies of GRBs. XRFs are empirically defined by a greater fluence (time-integrated flux) in the X-ray band $(2-30 \mathrm{keV})$ than in the gamma-ray band $(30-400 \mathrm{keV})$. This classification is also relevant for the investigation of GRB relations since some of them become stronger or weaker by introducing different GRB categories, see sec. 3.1.

One of the historical models used to explain the GRB phenomenon is the "fireball" model (Wijers et al. 1997; Mészáros 1998, 2006) in which a compact central engine (either the collapsed core of a massive star or the merger product of a neutron star binary) launches a highly relativistic, and jetted electron/positron/baryon plasma. Interactions of blobs within the jet are believed to produce the prompt emission, which consists of high photon energies such as gamma rays and hard X-rays. Instead, the interaction of the jet with the ambient material causes the afterglow phase, namely a long lasting multi-wavelength emission (X-ray, optical and sometimes also radio), which follows the prompt. However, problems in explaining the light curves within this model have been shown by Willingale et al. (2007), hereafter W07. More specifically, for $\sim 50 \%$ of GRBs, the observed afterglow is in agreement with the model, but for the rest, the temporal and spectral indices do not conform and are suggestive of continued late energy injection. The difficulty of the standard fireball models appeared when Swift ${ }^{1}$ observations had revealed a more complex behaviour of the light curves (O'Brien et al. 2006; Sakamoto et al. 2007; Zhang et al. 2007b) than in the past and pointed out that GRBs often follow "canonical" light curves (Nousek et al. 2006). In fact, the light curves can be divided into two, three and even more segments. The second segment, when it is flat, is called plateau emission. X-ray plateaus can be interpreted as occurring due to an accreting black hole (BH) (Cannizzo and Gehrels 2009; Cannizzo et al. 2011; Kumar et al. 2008) or a top-heavy jet evolution (Duffell and MacFadyen 2015). In addition, the fact that a newly born magnetar could be formed either via the collapse of a massive star or during the merger of two neutron stars motivated the interpretation of the X-ray plateaus as resulting from the delayed injection of rotational energy $\left(\dot{E}_{r o t} \sim 10^{50}-10^{51} \mathrm{erg} \mathrm{s}^{-1}\right)$ from a fast spinning magnetar (Usov 1992; Zhang and Mészáros 2001; Dall’Osso et al. 2011; Metzger et al. 2011; Rowlinson and O'Brien 2012; Rowlinson et al. 2014; Rea et al. 2015). These models are summarized in sec. 3.1.1.

Therefore, in this context, the discovery of relations amongst relevant physical parameters between prompt and plateau phases is very important so as to use them as possible model discriminators. In fact, many theoretical models have been presented in the literature to explain the wide variety of observations, but each model has some advantages and drawbacks. The use of the phenomenological relations corrected for selection biases can boost the understanding of the mechanism responsible for such emissions. Moreover, being observed at much larger redshift range than the SNe, it has long been tempting to consider GRBs as useful cosmological probes, extending the redshift range by almost an order of a magnitude further than the available SNe Ia, observed up to $z=2.26$ (Rodney et al. 2015). Indeed, GRBs are observed up to redshift $z=9.4$ (Cucchiara et al. 2011), which is much more distant than SNe Ia, and, therefore, they can help to understand the nature of the dark energy (DE), which is the main goal of modern cosmology, and determine the evolution of the equation of state (EoS), $w$, at very high $z$. So far, the most robust standard candles are the SNe Ia which, by being excellent distance indicators, provide a unique probe for measuring the expansion history of the Universe whose discovery has been awarded the Nobel Prize in 2011 (Riess et al. 1998; Perlmutter et al. 1998). Up-to-date, $w$ has been measured to be -1 within $5 \%$ of the Einstein's cosmological constant, $\Omega_{\Lambda}$, the pure vacuum energy. Measurement of the Hubble constant, $H_{0}$, provides another constraint on $w$ when combined with Cosmic Microwave Background Radiation (CMBR) and Baryon Acoustic Oscillation (BAO) measurements (Weinberg et al. 2013). Therefore, the use of other estimates provided by GRBs would be helpful to confirm further and/or

\footnotetext{
1 The Swift satellite was launched in 2004. With the instruments on board, the Burst Alert Telescope (BAT, divided in four standard channels 15-25; 25-50; 50-100; 100-150 keV), the X-Ray Telescope (XRT, 0.3-10 keV), and the Ultra-Violet/Optical Telescope (UVOT, $170-650 \mathrm{~nm}$ ), Swift provides a rapid follow-up of the afterglows in several wavelengths with better coverage than previous missions.
} 
constrain the ranges of values of $H_{0}$. However, different from the SNe Ia, which originate from white dwarves reaching the Chandrasekhar limit and always releasing the same amount of energy, GRBs cannot yet be considered standard candles with their isotropic energies spanning over 8 orders of magnitude. Therefore, finding out universal relations among observable properties can help to standardize their energetics and/or luminosities. It is for this reason that the study of GRB relations is relevant for both understanding the GRB emission mechanism, for finding a good distance indicator and for estimating the cosmological parameters at high $z$.

Until now, for cosmological purposes, the most used relations are the prompt emission relations: Amati (Amati et al. 2002) and Ghirlanda relations (Ghirlanda et al. 2004). The scatter of these relations is significantly reduced providing constraints on the cosmological parameters, see Ghirlanda et al. (2006) and Ghirlanda (2009) for details. By adopting a maximum likelihood approach which allows for correct quantification of the extrinsic scatter of the relation, Amati et al. (2008) constrained the matter density $\Omega_{M}$ (for a flat Universe) to 0.04-0.40 (68\% confidence level, CL), with a best-fit value of $\Omega_{M} \sim 0.15$, and exclude $\Omega_{M}=1$ at $>99.9 \%$ CL. Releasing the assumption of a flat Universe, they found evidence for a low value of $\Omega_{M}(0.04-0.50$ at $68 \%$ CL) as well as a weak dependence of the dispersion of the relation between the prompt peak energy in the $\nu F_{\nu}$ spectrum and the total gamma isotropic energy, $\log E_{\gamma, \text { peak }}-\log E_{\gamma, \text { iso }}$, on $\Omega_{\Lambda}$ (with an upper limit of $\Omega_{\Lambda} \sim 1.15$ at $90 \% \mathrm{CL}$ ). This approach makes no assumptions about the $\log E_{\gamma, p e a k}-\log E_{\gamma, \text { iso }}$ relation and it does not use other calibrators to set the normalization of the relation. Therefore, the treatment of the data is not affected by the so-called circularity problem (to calibrate the GRB luminosity relations for constraining cosmological models a particular cosmological model has to be assumed a priori) and the results are independent of those derived via SNe Ia (or other cosmological probes). Nowadays, the values of the cosmological parameters confirmed by measurements from the Planck Collaboration for the $\Lambda$ CDM model are $\Omega_{M}=0.3089 \pm 0.0062, \Omega_{\Lambda}=0.6911 \pm 0.0062$, and $H_{0}=67.74 \pm 0.46 \mathrm{Km} \mathrm{s}^{-1} \mathrm{Mpc}^{-1}$. For the investigation of the properties of DE, Amati and Della Valle (2013) showed the $68 \%$ CL contours in the $\Omega_{M}-\Omega_{\Lambda}$ plane obtained by assuming a sample of 250 GRBs expected shortly compared to those from other cosmological probes such as SNe Ia, CMB and Galaxy Clusters.

They obtained the simulated data sets via Monte Carlo techniques by taking into account the slope, normalization, and dispersion of the observed $\log E_{\gamma, \text { peak }}-\log E_{\gamma, \text { iso }}$ relation, the observed $z$ distribution of GRBs and the distribution of the uncertainties in the measured values of $\log E_{\gamma, p e a k}$ and $\log E_{\gamma, i s o}$. These simulations indicated that with a sample of $250 \mathrm{GRBs}$, the accuracy in measuring $\Omega_{M}$ would be comparable to that currently provided by SNe data. In addition, they reported the estimates of $\Omega_{M}$ and the parameter of the DE EoS, $w_{0}$, derived from the present and expected future samples. They assumed that the $\log E_{\gamma, \text { peak }}-\log E_{\gamma, \text { iso }}$ relation is calibrated with a $10 \%$ accuracy by using, e.g., the luminosity distances provided by SNe Ia and the self-calibration of the relation with a large enough number of GRBs lying within a narrow range of $\mathrm{z}(\Delta z \sim 0.1-0.2)$. Generally speaking, as the number of GRBs in each redshift bin increases, also the feasibility and accuracy of the self-calibration of GRB relations will improve. For a review on GRB prompt relations, see Dainotti et al. (2016b).

Even though the errors on $\Omega_{M}$ obtained in Amati and Della Valle (2013) may lead to GRBs as promising standard candles, because they are almost comparable with SNe (0.06 for GRBs versus 0.04 for $\mathrm{SNe}$, as provided for the $\mathrm{SNe}$ sample by Betoule et al. 2014 and Calcino and Davis 2017), these results show that $\Omega_{M}$ has an error which is 20 times larger then the value obtained by Planck. Thus, GRBs in a near future can be comparable with SNe Ia, but not likely with Planck. On the other hand, there is discrepancy among the values of $H_{0}$ computed by CMB and SNe (Planck Collaboration et al. 2016) and thus adding a new effective cosmological probe as GRBs can help to cast light on this discrepancy and break the degeneracy among several cosmological parameters.

It is clear from this context that selection biases play a major and crucial role even for the close-by probes such as SNe Ia in determining the correct cosmological parameters. This problem is more relevant for GRBs, which are particularly affected by the Malmquist bias effect (Malmquist 1920, Eddington 1940) that favours the brightest objects against faint ones at large distances. Therefore, it is necessary to investigate carefully the problem of selection effects and how to overcome them before using GRB relations as distance estimators, as cosmological probes, and as model discriminators. This is indeed the major aim of this review. Besides, this work is useful, especially for those embarking on the study of GRB relations, because it aims at constituting a brief, but a complete compendium of afterglow and prompt-afterglow relations.

The review is organized as follows: in section 2, we explain the nomenclature and definitions in all review, in sections 3 and 4, we analyze the relations between the afterglow parameters and between parameters of both the prompt and afterglow phases. In section 5 , we describe how these relations can be affected by selection biases. In section 6 , we present how to obtain a redshift estimator and in section 7, we report the use of the Dainotti relation as an example of GRB application as a cosmological tool. Finally, in section 8, we briefly summarize some findings about the physical 
models and the cosmological usage of the analyzed relations, while in the last section we draw our conclusions.

\section{NOTATIONS}

For clarity, we report a summary of the nomenclature adopted in the review.

- $L, E, F, S$, and $T$ indicate the luminosity, the energy, the flux, the fluence and the time which can be observed in several wavelengths, denoted with the first subscript, and at different times or part of the light curve, denoted instead with the second subscript. In addition, with $\alpha, \beta$ and $\nu$, we represent the temporal and spectral decay indices and the frequencies.

More specifically:

- $T_{X, a}$ and $T_{O, a}$ denote the time in the X-ray at the end of the plateau and the same time, but in the optical wavelength respectively. $F_{X, a}$ are $F_{O, a}$ are their respective fluxes, while $L_{X, a}$ and $L_{O, a}$ are their respective luminosities. An approximation of the energy of the plateau is $E_{X, p l a t e a u}=\left(L_{X, a} \times T_{X, a}^{*}\right)$, see the left panel of Fig. 1.

- $T_{O, p e a k}$ and $T_{X, f}$ are the peak time in the optical and the time since ejection of the pulse. $L_{O, p e a k}$ and $L_{X, f}$ are their respective luminosities. $F_{O, p e a k}$ is the respective flux of $T_{O, p e a k}$.

- $T_{X, p e a k}$ is the peak time in the X-ray and $F_{X, p e a k}$ and $L_{X, p e a k}$ are its flux and luminosity respectively.

- $T_{X, p}$ and $T_{X, t}$ are the time at the end of the prompt emission within the W07 model and the time at which the flat and the step decay behaviours of the light curves join respectively.

- $T_{90}$ and $T_{45}$ are the times in which the $90 \%$ (between $5 \%$ and $95 \%$ ) and $45 \%$ (between $5 \%-50 \%$ ) of radiation is emitted in the prompt emission respectively.

- $\tau_{l a g}$ and $\tau_{R T}$ are the differences in arrival time to the observer of the high energy photons and low energy photons and the shortest time over which the light curve increases by the $50 \%$ of the peak flux of the pulse.

- $L_{X, 200 \mathrm{~s}}, L_{X, 10}, L_{X, 11}, L_{X, 12}, L_{X, 1 \mathrm{~d}}$ and $L_{O, 200 \mathrm{~s}}, L_{O, 10}, L_{O, 11}, L_{O, 12}, L_{O, 1 \mathrm{~d}}$ are the X-ray and optical luminosities at $200 \mathrm{~s}$, at 10,11, 12 hours and at 1 day respectively; $L_{O, 100 s}, L_{O, 1000 s}, L_{O, 10000 s}, L_{O, 7}$ are the optical luminosity at $100 \mathrm{~s}, 1000 \mathrm{~s}, 10000 \mathrm{~s}$ and 7 hours; $L_{\gamma, \text { iso }}$ and $L_{L}\left(\nu, T_{X, a}\right)$ are the isotropic prompt emission mean luminosity and the optical or X-ray luminosity of the late prompt emission at the time $T_{X, a}$.

- $F_{X, 11}, F_{X, 1 \mathrm{~d}}$ and $F_{O, 11}, F_{O, 1 \mathrm{~d}}$ are the X-ray and optical fluxes at 11 hours and at 1 day respectively; $F_{\gamma, p r o m p t}$, $F_{X, \text { afterglow }}$ are the gamma-ray flux in the prompt and the X-ray flux in the afterglow respectively. $E_{\gamma, \text { prompt }}$ and $E_{X, \text { afterglow }}$ are their respective isotropic energies and $L_{\gamma, p r o m p t}$ and $L_{X, \text { afterglow }}$ are the respective luminosities. $S_{\gamma, p r o m p t}$ indicates the prompt fluence in the gamma band correspondent to the rest frame isotropic prompt energy $E_{\gamma, \text { prompt }}$.

- $E_{O, \text { afterglow }}, E_{\gamma, \text { iso }}$ and $E_{X, f}$ are the optical isotropic energy in the afterglow phase, the total gamma isotropic energy and the prompt emission energy of the pulse.

- $E_{k, a f t}, E_{\gamma, p e a k}$ and $E_{\gamma, c o r}$ are the isotropic kinetic afterglow energy in X-ray, the prompt peak energy in the $\nu F_{\nu}$ spectrum and the isotropic energy corrected for the beaming factor.

- $\alpha_{X, a}, \alpha_{O,>200 \mathrm{~s}}, \alpha_{X,>200 \mathrm{~s}}, \alpha_{\nu, f l}$ and $\alpha_{\nu, s t}$ are the X-ray temporal decay index in the afterglow phase, in the optical after $200 \mathrm{~s}$, in the X-ray after $200 \mathrm{~s}$ and the optical or X-ray flat and steep temporal decay indices respectively.

- $\beta_{X, a}, \beta_{O X, a}$ and $\beta_{O,>200 s}$ are the spectral index of the plateau emission in X-ray, the optical-to-X-ray spectral index for the end time of the plateau and the optical spectral index after $200 \mathrm{~s}$.

- $\nu_{X}, \nu_{O}, \nu_{c}, \nu_{m}$ are the X-ray and optical frequencies, and the cooling and the peak frequencies of the synchrotron radiation.

All the time quantities described above are given in the observer frame, while with the upper index $*$ we denote in the text the observables in the GRB rest frame. The rest frame times are the observed times divided by the cosmic time expansion, for example, $T_{X, a}^{*}=T_{X, a} /(1+z)$ denotes the rest frame time at the end of the plateau emission. 

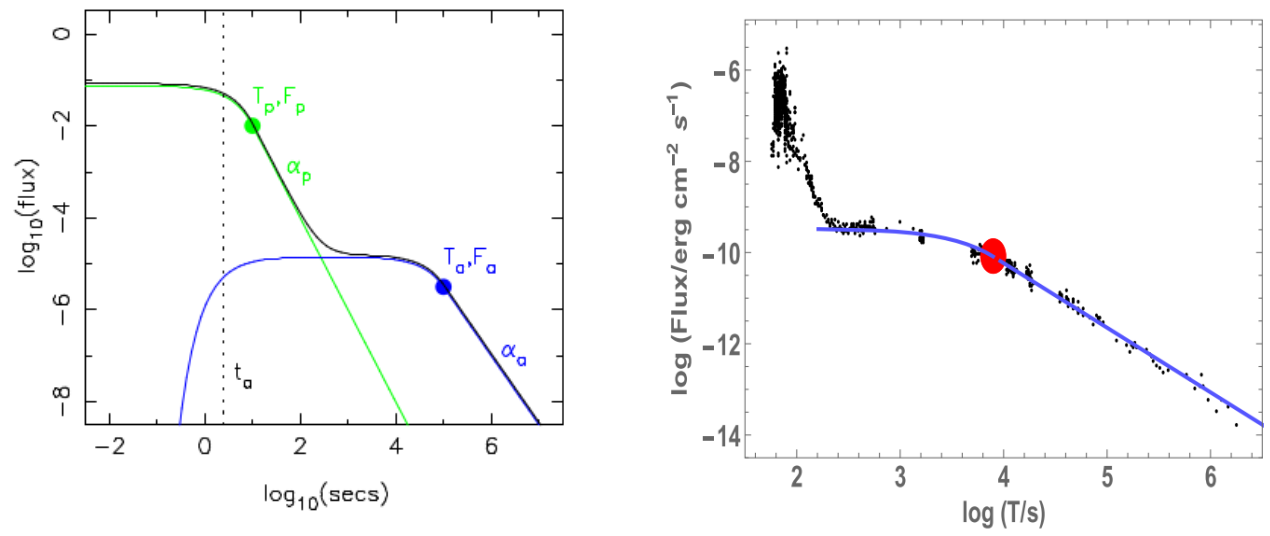

Figure 1. Left panel: the functional form of the fitting model from Willingale et al. (2007). Right panel: the observed light curve for GRB 061121 with the best-fit W07 model superimposed from Dainotti et al. (2016a). The red dot marks the end of the flat plateau phase in the X-ray afterglow $\left(T_{X, a}, F_{X, a}\right)$. A similar configuration appears in the optical range.

In the following table we will give a list of the abbreviations/acronyms used through the text:

\begin{tabular}{|c|c|}
\hline Abbreviation & Meaning \\
\hline $\mathrm{DE}$ & Dark Energy \\
\hline EoS & Equation of State \\
\hline CL & Confidence Level \\
\hline IC & Intermediate Class GRB \\
\hline SGRB & Short GRB \\
\hline LGRB & Long GRBs \\
\hline SGRBsEE & Short GRBs with extended emission \\
\hline XRFs & X-ray Flashes \\
\hline $\mathrm{SNe}$ & Supernovae \\
\hline $\mathrm{BH}$ & Black Hole \\
\hline $\mathrm{z}$ & redshift \\
\hline FS & Forward Shock \\
\hline $\mathrm{RS}$ & Reverse Shock \\
\hline$H_{0}$ & Hubble constant \\
\hline$\Omega_{M}$ & Matter density in $\Lambda$ CDM model \\
\hline$\Omega_{\Lambda}$ & Dark Energy density in $\Lambda \mathrm{CDM}$ model \\
\hline$\Omega_{k}$ & curvature in $\Lambda \mathrm{CDM}$ model \\
\hline$\sigma_{\log L_{X, a}}$ & error on the luminosity \\
\hline$\sigma_{\log T_{X, a}^{*}}$ & error on the time \\
\hline E4 & sample with $\sigma_{E}=\left(\sigma_{\log L_{X, a}}^{2}+\sigma_{\log T_{X, a}^{*}}^{2}\right)^{1 / 2}<4$ \\
\hline E0095 & sample with $\sigma_{E}=\left(\sigma_{\log L_{X, a}}^{2}+\sigma_{\log T_{X, a}^{*}}^{2}\right)^{1 / 2}<0.095$ \\
\hline W07 & Willingale et al. (2007) \\
\hline$\Gamma$ & Lorentz Factor \\
\hline$V$ & Variability of the GRB light curve \\
\hline$h$ & Hubble constant divided by 100 \\
\hline$w_{0}, w_{a}$ & coefficients of the DE $\operatorname{EoS} w(z)=w_{0}+w_{a} z(1+z)^{-1}$ \\
\hline HD & Hubble Diagram \\
\hline $\mathrm{a}$ & normalization of the relation \\
\hline $\mathrm{b}$ & slope of the relation \\
\hline$\sigma_{\text {int }}$ & intrinsic scatter of the relation \\
\hline$b_{\text {int }}$ & intrinsic slope of the relation \\
\hline
\end{tabular}

Table 1. Table with abbreviations. 


\section{THE AFTERGLOW RELATIONS}

Several relations appeared in literature relating only parameters in the afterglow, such as the $L_{X}\left(T_{a}\right)-T_{X, a}^{*}$ relation (Dainotti et al. 2008) and similar ones in the optical and X-ray bands such as the unified $L_{X}\left(T_{a}\right)-T_{X, a}^{*}$ and $L_{O, a}-T_{O, a}^{*}$ (Ghisellini et al. 2009) and the $L_{O, 200 \mathrm{~s}}-\alpha_{O,>200 \mathrm{~s}}$ relations (Oates et al. 2012).

\subsection{The Dainotti relation $\left(L_{X}\left(T_{a}\right)-T_{X, a}^{*}\right)$}

The first relation to shed light on the plateau properties has been the $L_{X}\left(T_{a}\right)-T_{X, a}^{*}$ one, hereafter also referred as LT. The phenomenon is an anti-relation between the X-ray luminosity at the end of the plateau, $L_{X}\left(T_{a}\right)$, and the time in the X-ray at the end of the plateau, $T_{X, a}^{*}$, for simplicity of notation we will refer to $L_{X}\left(T_{a}\right)$ as $L_{X, a}$.

It was discovered by Dainotti et al. (2008) using 33 LGRBs detected by the Swift satellite in the X-ray energy band observed by XRT. Among the 107 GRBs fitted by W07 phenomenological model, shown in the left panel of Fig. 1, only the GRBs that have a good spectral fitting of the plateau and firm determination of $z$ have been chosen. The functional form of the LT relation obtained is the following:

$$
\log L_{X, a}=a+b \times \log T_{X, a}^{*}
$$

with a normalization $a=48.54$, a slope $b=-0.74_{-0.19}^{+0.20}$, an intrinsic scatter, $\sigma_{i n t}=0.43$ and a Spearman correlation coefficient $^{2} \rho=-0.74 . L_{X, a}$ in the Swift XRT passband, $\left(E_{\min }, E_{\max }\right)=(0.3,10) \mathrm{keV}$, has been computed from the following equation:

$$
L_{X, a}(z)=4 \pi D_{L}^{2}\left(z, \Omega_{M}, h\right) F_{X, a} \times K
$$

where $D_{L}\left(z, \Omega_{M}, h\right)$ represents the GRB luminosity distance for a given $z, F_{X, a}$ indicates the flux in the X-ray at the end of the plateau, and $K=\frac{1}{(1+z)^{(1-\beta X, a)}}$ denotes the K-correction for cosmic expansion (Bloom et al. 2001). This anti-relation shows that the shorter the plateau duration, the more luminous the plateau. Since the ratio between the errors on both variables is close to unity, it means that both errors need to be considered and the Marquardt Levenberg algorithm is not the best fitting method to be applied in this circumstance. Therefore, a Bayesian approach (D'Agostini 2005) needs to be considered. This method takes into account the errors of both variables and an intrinsic scatter, $\sigma_{i n t}$, of unknown nature. However, the results of both the D'Agostini method and the Marquardt Levenberg algorithm are comparable. Due to the higher accuracy of the first method from now on the authors prefer this technique in their papers. Evidently, the tighter the relation, the better the chances to constrain the cosmological parameters. With this specific challenge in mind, a subsample of bursts has been chosen with particular selection criteria both on luminosity and time, namely $\log L_{X, a}>45$ and $1 \leq \log T_{X, a}^{*} \leq 5$. After this selection has been applied, a subsample of 28 LGRBs was obtained with $\left(a, b, \sigma_{\text {int }}\right)=(48.09,-0.58 \pm 0.18,0.33)$, thus reducing considerably the scatter.

In agreement with these results, through the analysis of the late prompt phase in optical and X-ray light curves of 33 LGRBs, also Ghisellini et al. (2009) found a common observational model for optical and X-ray light curves with the same value for the slope, $b=-0.58_{-0.18}^{+0.18}$, obtained by Dainotti et al. (2008) when the time is limited between $1 \leq \log T_{X, a}^{*} \leq 5$.

Instead, Dainotti et al. (2010) from a sample of $62 \mathrm{LGRBs}$ found $b=-1.06_{-0.28}^{+0.27}$, while for the 8 IC GRBs pointed out a much steeper relation $\left(b=-1.72_{-0.21}^{+0.22}\right)$. Finally, taking into account the errors on luminosity $\left(\sigma_{\log L_{X, a}}\right)$ and time $\left(\sigma_{\log T_{X, a}^{*}}\right)$, the 8 GRBs with the smallest errors were defined as the ones with $\sigma_{E}=\left(\sigma_{\log L_{X, a}}^{2}+\sigma_{\log T_{X, a}^{*}}^{2}\right)^{1 / 2}<0.095$. For this subsample, Dainotti et al. (2010) found a slope $-1.05_{-0.20}^{+0.19}$, see Fig. 2, the right panel of Fig. 3 and Table 2. Similar to Dainotti et al. (2010), also Bernardini et al. (2012a) and Sultana et al. (2012), with a sample of 64 and 14 LGRBs respectively, found a slope $b \approx-1$, for details see Table 2 .

Expanding the sample again to 77 LGRBs, Dainotti et al. (2011a) discovered a relation with $b=-1.20_{-0.30}^{+0.27}$. Later, Mangano et al. (2012), considering in their sample of 50 LGRBs those GRBs with no visible plateau phase and employing a broken power law as a fitting model, found a steeper slope $\left(b=-1.38_{-0.16}^{+0.16}\right)$. Thus, from all these analyses it is clear that a steepening of the slope has been observed when the sample size is increased.

Therefore, before going further with additional analysis, Dainotti et al. (2013a) decided to show how selection biases can influence the slope of the relation. They showed that the steepening of the relation results from selection biases, while the intrinsic slope of the relation is $b=-1.07_{-0.14}^{+0.09}$, see section 5. Summarizing, Dainotti et al. (2013a) with a

\footnotetext{
${ }^{2}$ A computation of statistical dependence between two variables stating how good the relation between these variables can be represented employing a monotonic function. It assumes a value between -1 and +1 .
} 

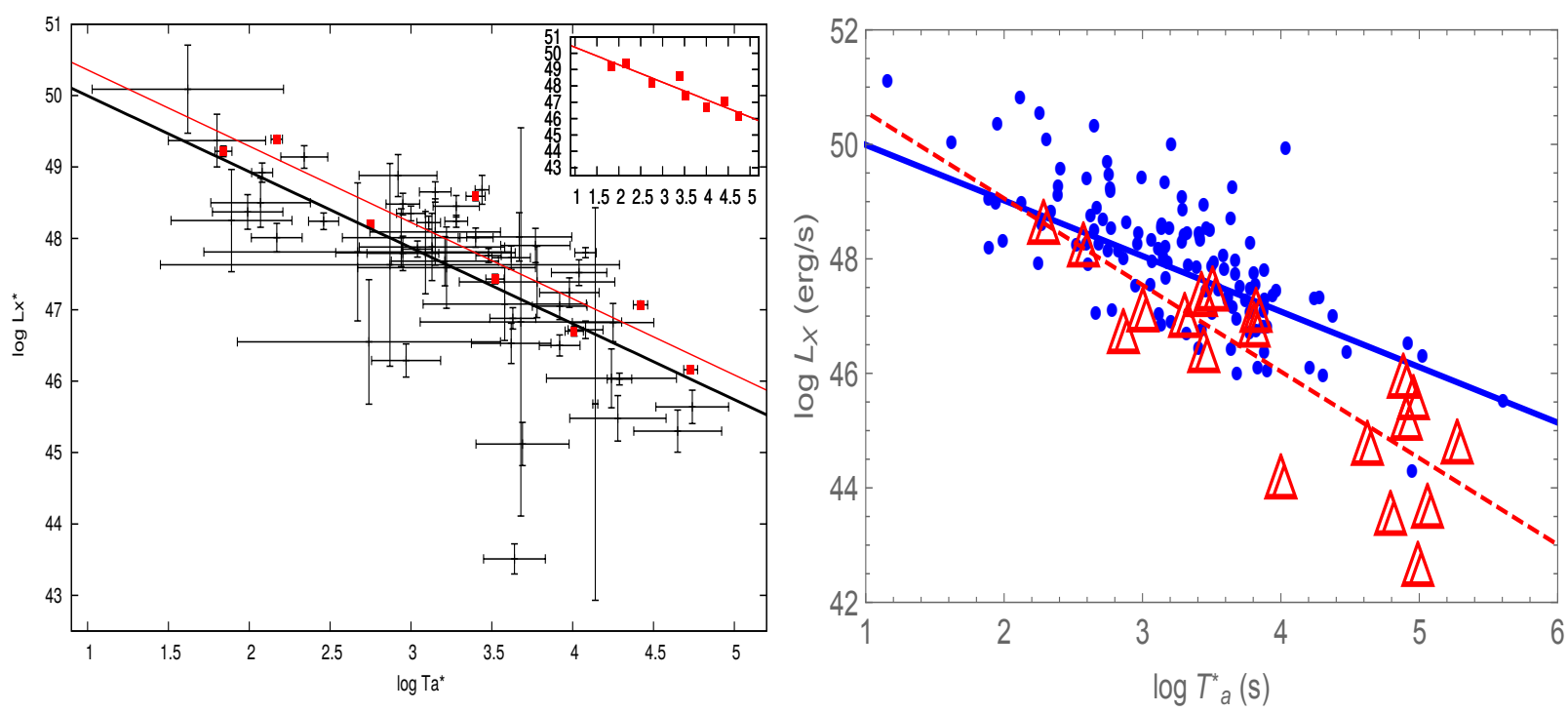

Figure 2. Left panel: $\log L_{X, a}$ (equivalent to $\log L_{X}^{*}$ in this plot) vs. $\log T_{X, a}^{*}$ for 62 long afterglows with the error energy parameter $\sigma_{E}<4$, and the best fitted relation line in black, from Dainotti et al. (2010). The red line fitted to the 8 lowest error (red) points produces an upper envelope of the full data set. The upper envelope points with the best fitted line are separately presented in an inset panel. Right panel: LONG-NO-SNe 128 GRBs (blue points fitted with a solid blue line) and the 19 events from LONG-SNe (red empty triangles) fitted with a red dashed line from Dainotti et al. (2016c)

sample of 101 GRBs, confirmed the previous results from Dainotti et al. (2010), as well as Rowlinson et al. (2014), with a data set of 159 GRBs.
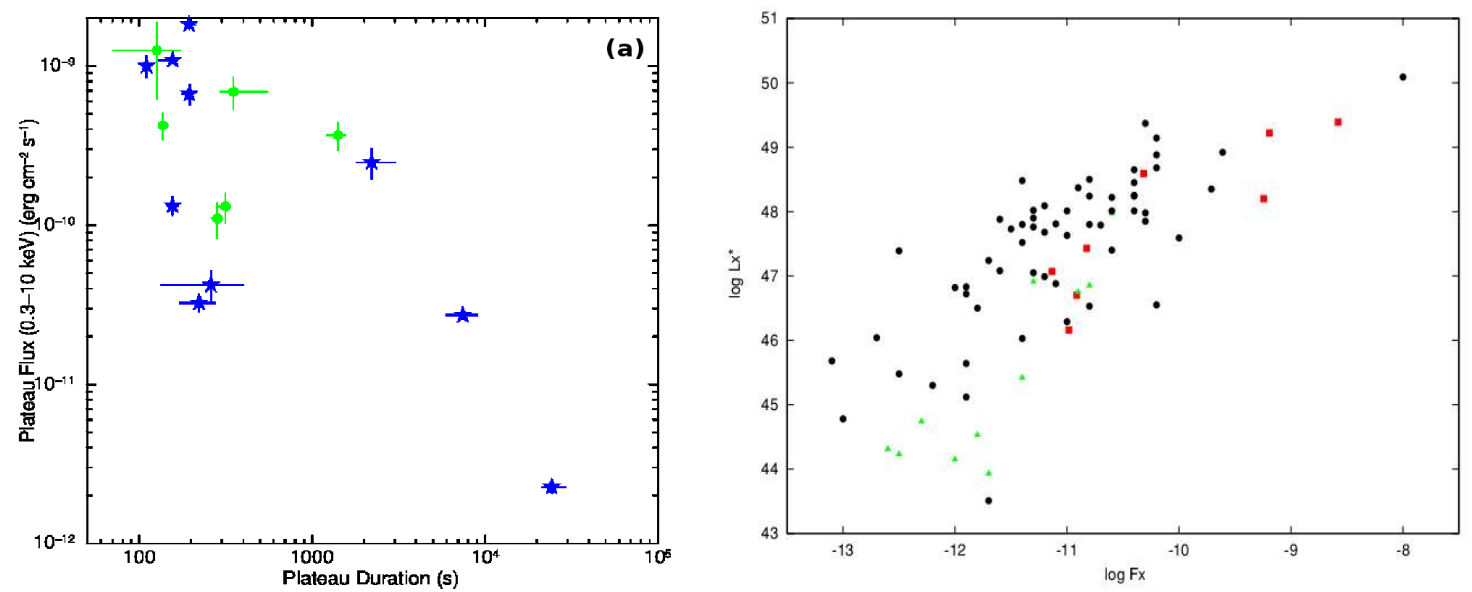

Figure 3. Left panel: the plateau flux versus the plateau duration for a sample of 22 SGRBs from Rowlinson et al. (2013). Blue stars are GRBs with two or more breaks in their light curves, while green circles have one break. Right panel: $\log L_{X, a}$ versus $\log F_{X, a}$ for the full GRB sample from Dainotti et al. (2010). The 8 upper envelope points are shown as red squares, while the IC GRBs are represented by green triangles.

Dainotti et al. (2015b) also confirmed previous results of Dainotti et al. (2013a) but with a larger sample of 123 LGRBs. All the samples discussed are observed by SWIFT/XRT.

In the context of reducing the scatter of the LT relation, Del Vecchio et al. (2016) investigated the temporal decay indices $\alpha_{X, a}$ after the plateau phase for a sample of 176 GRBs detected by Swift within two different models: a simple power law, considering the decaying phase after the plateau phase, and the W07 one. It is pointed out that the results are independent of the chosen model. It was checked if there are some common characteristics in GRBs phenomena that can allow them to be used as standardizable candles like SNe Ia and to obtain some constraints revealing which is the best physical interpretation describing the plateau emission. The interesting result is that the LT relation for the low and high luminosity GRBs seems to depend differently on the $\alpha_{X, a}$ parameter, thus possibly implying a diverse density medium. 


\begin{tabular}{|c|c|c|c|c|c|c|}
\hline Author & $\mathrm{N}$ & Type & Slope & Norm & Corr.coeff. & $\mathrm{P}$ \\
\hline Dainotti et al. (2008) & 28 & $1<T_{X, a}^{*}<5$ & $-0.58_{-0.18}^{+0.18}$ & 48.09 & -0.80 & $1.6 \times 10^{-7}$ \\
\hline Dainotti et al. (2008) & 33 & All GRBs & $-0.74_{-0.19}^{+0.20}$ & 48.54 & -0.74 & $10^{-9}$ \\
\hline Cardone et al. (2009) & 28 & L & $-0.58_{-0.18}^{+0.18}$ & 48.09 & -0.74 & $10^{-9}$ \\
\hline Ghisellini et al. (2009) & 33 & $\mathrm{~L}$ & $-0.58_{-0.18}^{+0.18}$ & 48.09 & -0.74 & $10^{-9}$ \\
\hline Cardone et al. (2010) & 66 & $\mathrm{~L}$ & $-1.04_{-0.22}^{+0.23}$ & $50.22_{-0.76}^{+0.77}$ & -0.68 & $7.6 \times 10^{-9}$ \\
\hline Dainotti et al. (2010) & 62 & $\mathrm{~L}$ & $-1.06_{-0.28}^{+0.27}$ & $51.06_{-1.02}^{+1.02}$ & -0.76 & $1.85 \times 10^{-11}$ \\
\hline Dainotti et al. (2010) & 8 & high luminosity & $-1.05_{-0.20}^{+0.19}$ & $51.39_{-0.90}^{+0.90}$ & -0.93 & $1.7 \times 10^{-2}$ \\
\hline Dainotti et al. (2010) & 8 & IC & $-1.72_{-0.21}^{+0.22}$ & $52.57_{-1.04}^{+1.04}$ & -0.66 & $7.4 \times 10^{-2}$ \\
\hline Dainotti et al. (2011a) & 77 & $\mathrm{~L}$ & $-1.20_{-0.30}^{+0.27}$ & $51.04_{-0.30}^{+0.27}$ & -0.69 & $7.7 \times 10^{-8}$ \\
\hline Sultana et al. (2012) & 14 & $\mathrm{~L}$ & $-1.10_{-0.03}^{+0.03}$ & $51.57_{-0.10}^{+0.10}$ & -0.88 & $10^{-5}$ \\
\hline Bernardini et al. (2012) & 64 & $\mathrm{~L}$ & $-1.06_{-0.06}^{+0.06}$ & 51.06 & -0.68 & $7.6 \times 10^{-9}$ \\
\hline Mangano et al. (2012) & 50 & $\mathrm{~L}$ & $-1.38_{-0.16}^{+0.16}$ & $52.2_{-0.06}^{+0.06}$ & -0.81 & $2.4 \times 10^{-10}$ \\
\hline Dainotti et al. (2013a) & 101 & ALL intrinsic & $-1.07_{-0.14}^{+0.09}$ & 52.94 & -0.74 & $10^{-18}$ \\
\hline Dainotti et al. (2013b) & 101 & All GRBs & $-1.32_{-0.17}^{+0.18}$ & $52.8_{-0.3}^{+0.9}$ & -0.74 & $10^{-18}$ \\
\hline Dainotti et al. (2013b) & 101 & without short & $-1.27_{-0.26}^{+0.18}$ & 52.94 & -0.74 & $10^{-18}$ \\
\hline Dainotti et al. (2013b) & 101 & simulated & $-1.52_{-0.24}^{+0.04}$ & $53.27_{-0.48}^{+0.54}$ & -0.74 & $10^{-18}$ \\
\hline Postnikov et al. (2014) & 101 & $\mathrm{~L}(z<1.4)$ & $-1.51_{-0.27}^{+0.26}$ & $53.27_{-0.48}^{+0.54}$ & -0.74 & $10^{-18}$ \\
\hline Rowlinson et al. (2014) & 159 & intrinsic & $-1.07_{-0.14}^{+0.09}$ & 52.94 & -0.74 & $10^{-18}$ \\
\hline Rowlinson et al. (2014) & 159 & observed & $-1.40_{-0.19}^{+0.19}$ & $52.73_{-0.52}^{+0.52}$ & -0.74 & $10^{-18}$ \\
\hline Rowlinson et al. (2014) & 159 & simulated & $-1.30_{-0.03}^{+0.03}$ & $52.73_{-0.52}^{+0.52}$ & -0.74 & $10^{-18}$ \\
\hline Dainotti et al (2015) & 123 & $\mathrm{~L}$ & $-0.90_{-0.17}^{+0.19}$ & $51.14_{-0.58}^{+0.58}$ & -0.74 & $10^{-15}$ \\
\hline Dainotti et al. (2016c) & 19 & $\mathrm{~L}-\mathrm{SNe}$ & $-1.5_{-0.3}^{+0.3}$ & $51.85_{-0.94}^{+0.94}$ & -0.83 & $5 \times 10^{-6}$ \\
\hline
\end{tabular}

Table 2. Summary of the LT relation. All the measurements are performed by the Swift XRT Telescope. The first column represents the authors, the second one the number of GRBs in the used sample, the third one the GRB type ( $\mathrm{S}=\mathrm{Short}$, L=Long, IC=Intermediate), the fourth and the fifth ones are the slope and normalization of the relation and the last two columns are the correlation coefficient and the chance probability, $\mathrm{P}$.

Continuing the search for a standard set of GRBs, Dainotti et al. (2016c) analyzed 176 GRB afterglow plateaus observed by Swift with known redshifts which revealed that the subsample of LGRBs associated with SNe (LONG$\mathrm{SNe}$ ) presents a very high correlation coefficient for the LT relation. They investigated the category of LONG GRBs associated spectroscopically with SNe in order to compare the LT correlation for this sample with the one for LGRBs for which no associated SN has been observed (hereafter LONG-NO-SNe, 128 GRBs). They checked if there is a difference among these subsamples. They adopted first a non-parametric statistical method, the Efron and Petrosian (1992) one, to take into account redshift evolution and check if and how this effect may steepen the slope for the LONG-NO-SNe sample. This procedure is necessary due to the fact that this sample is observed at much higher redshift than the GRB-SNe sample. Therefore, removing selection bias is the first step before any comparison among samples observed at different redshifts could be properly performed. They have demonstrated that there is no evolution for the slope of the LONG-NO-SNe sample and no evolution is expected for the LONG-SNe sample. The difference among the slopes is statistically significant with the probability $\mathrm{P}=0.005$ for LONG-SNe. This possibly suggests that the LONG-SNe with firm spectroscopic features of the SNe associated might not require a standard energy reservoir in the plateau phase unlike the LONG-NO-SNe. Therefore, this analysis may open new perspectives in future theoretical investigations of the GRBs with plateau emission and associated with SNe. They also discuss how much this difference can be due to the jet opening angle effect. The difference between the SNe-LONG $(\mathrm{A}+\mathrm{B})$ and LONG-NO-SNe sample is only statistically significant at the $10 \%$ level when we consider the beaming correction. Thus, one can argue that the difference in slopes can be partially due to the effect of the presence of low luminosity GRBs in the LONG-SNe sample that are not corrected for beaming. However, the beaming corrections are not very accurate due to indeterminate jet opening angles, so the debate remains open and it can only be resolved when we will gather more data.

In Table 2, we report a summary of the parameters $a$ and $b$ with $\rho$ and $P$ for the LT relation. In conclusion, the most reliable parameters for this relation are those from Dainotti et al. (2013a), because they have demonstrated that the intrinsic slope not affected by selection biases is determined to be -1 as computed through the Efron and Petrosian (EP) method. 


\subsubsection{Physical interpretation of the Dainotti relation $\left(L_{X}\left(T_{a}\right)-T_{X, a}^{*}\right)$}

Here, we revise the theoretical interpretation of the LT relation, which is based mainly on the accretion (Cannizzo and Gehrels 2009; Cannizzo et al. 2011) and the magnetar models (Zhang and Mészáros 2001; Dall'Osso et al. 2011; Rowlinson and O'Brien 2012; Rowlinson et al. 2013, 2014).

The first one states that an accretion disc is created from the motion of the material around the GRB progenitor star collapsing towards its progenitor core. After it is compressed by the gravitational forces, the GRB emission takes place. For LGRBs, the early rate of decline in the initial steep decay phase of the light curve may provide information about the radial density distribution within the progenitor (Kumar et al. 2008).

Cannizzo and Gehrels (2009) predicted a steeper relation slope (-3/2) than the observed one $(\sim-1)$, which on the other hand is in good agreement with the prior emission model of Yamazaki (2009).

Later, Cannizzo et al. (2011), using a sample of 62 LGRBs and few SGRBs simulated the fall-back disks surrounding the BH. They found that a circularization radius of the mass around the $\mathrm{BH}$ with value $10^{10}-10^{11} \mathrm{~cm}$ can give an estimate for the plateau duration of around $10^{4} \mathrm{~s}$ for LGRBs maintaining the initial fall back mass at $10^{-4}$ solar masses $\left(M_{\odot}\right)$, see the left panel of Fig. 4. For SGRBs the radius is estimated to be $10^{8} \mathrm{~cm}$. The LT relation provides a lower limit for the accreting mass estimates $\Delta M \approx 10^{-4}$ to $10^{-3} M_{\odot}{ }^{3}$. From their results, it was claimed that the LT relation could be obtained if a typical energy reservoir in the fall-back mass is assumed, see the right panel of Fig. 4 . However, in their analysis the very steep initial decay following the prompt emission, which have been modelled by Lindner et al. (2010) as fall-back of the progenitor core, is not considered.
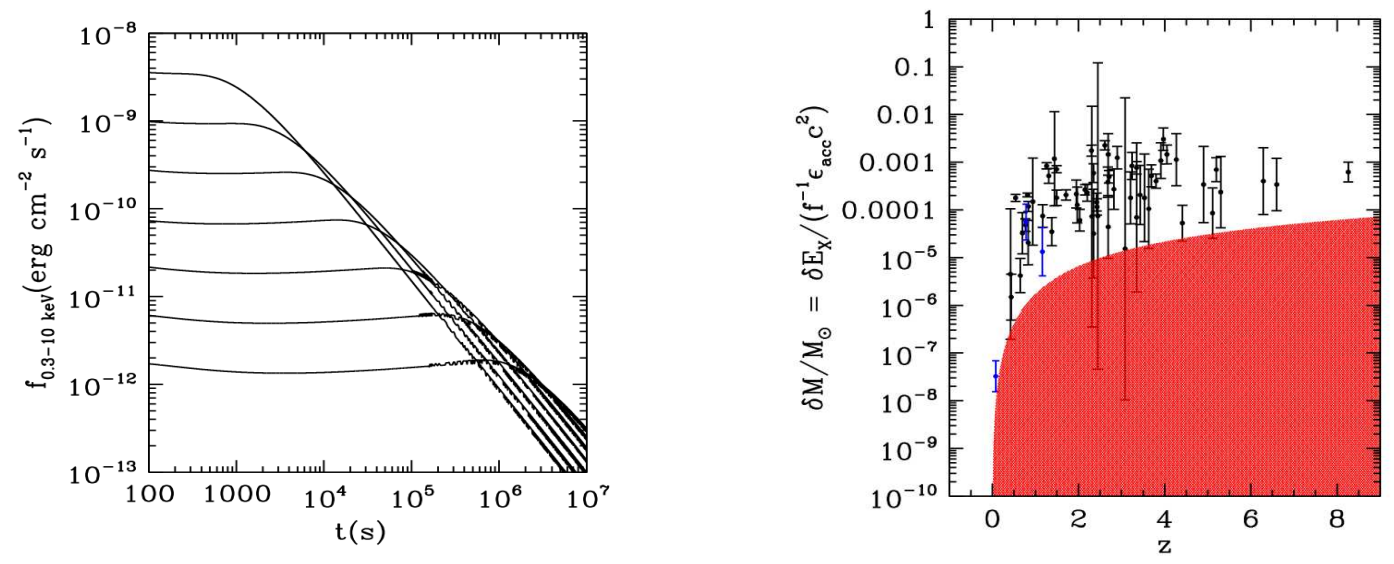

Figure 4. Left panel: model light curves for LGRB parameters from Cannizzo et al. (2011), keeping the starting fall-back disk mass constant at $10^{-4} M_{\odot}$ but changing the initial radius and normalization. Right panel: total accretion mass for the plateau + later decay phases of GRBs from Cannizzo et al. (2011), considering 62 LGRBs from Dainotti et al. (2010). The region in red represents a limiting XRT detection flux level $f_{\mathrm{II}}=10^{-12} \mathrm{erg} \mathrm{cm}^{-1} \mathrm{~s}^{-1}$ (assuming a plateau duration $t_{\mathrm{II}}=10^{4} \mathrm{~s}$ ) in order to study a plateau to sufficient accuracy. A beaming factor $f=1 / 300$ and a net efficiency for powering the X-ray flux $\epsilon_{\text {net }}=\epsilon_{\text {acc }} \epsilon_{X}=0.03$ were assumed.

Regarding the magnetar model, Zhang and Mészáros (2001) studied the effects of an injecting central engine on the GRB afterglow radiation, concentrating on a strongly magnetized millisecond pulsar. For specific starting values of rotation period and magnetic field of the pulsar, the afterglow light curves should exhibit an achromatic bump lasting from minutes to months, and the observation of such characteristics could set some limits on the progenitor models. More recently, Dall'Osso et al. (2011) investigated the energy evolution in a relativistic shock from a spinning down magnetar in spherical symmetry. With their fit of few observed Swift XRT light curves and the parameters of this model, namely a spin period of $(1-3 \mathrm{~ms})$, and high values of magnetic fields $\left(B \sim 10^{14}-10^{15} \mathrm{G}\right)$, they managed to well reproduce the properties of the shallow decay phase and the LT relation, see the left panel of Fig. 5 .

Afterward, Bernardini et al. (2012a) with a sample of 64 LGRBs confirmed, as previously founded by Dall'Osso et al. (2011), that the shallow decay phase of the GRB light curves and the LT relation can be well explained.

Then, Rowlinson and O'Brien (2012) and Rowlinson et al. (2013) pointed out that energy injection is a fundamental mechanism for describing the plateau emission of both LGRBs and SGRBs. In fact, the remnant of NS-NS mergers

3 This value can be derived considering the total inferred accretion mass $\Delta M / M=\Delta E_{X} / f^{-1} * \epsilon_{a c c} * c^{2}$ where $\mathrm{c}$ is the light speed, $\mathrm{f}$ is the X-ray afterglow beaming factor, $\epsilon_{a c c}$ is the efficiency of the accretion onto the $\mathrm{BH}$ and $E_{X}$ is the observed total energy of the plateau + later decay phases (the integral over time between $T_{X, t}$ and the end of afterglow, see Eq. 2 of W07). 
can form a magnetar, and indeed the origin of the majority of SGRBs is well explained through the energy injection by a magnetar.
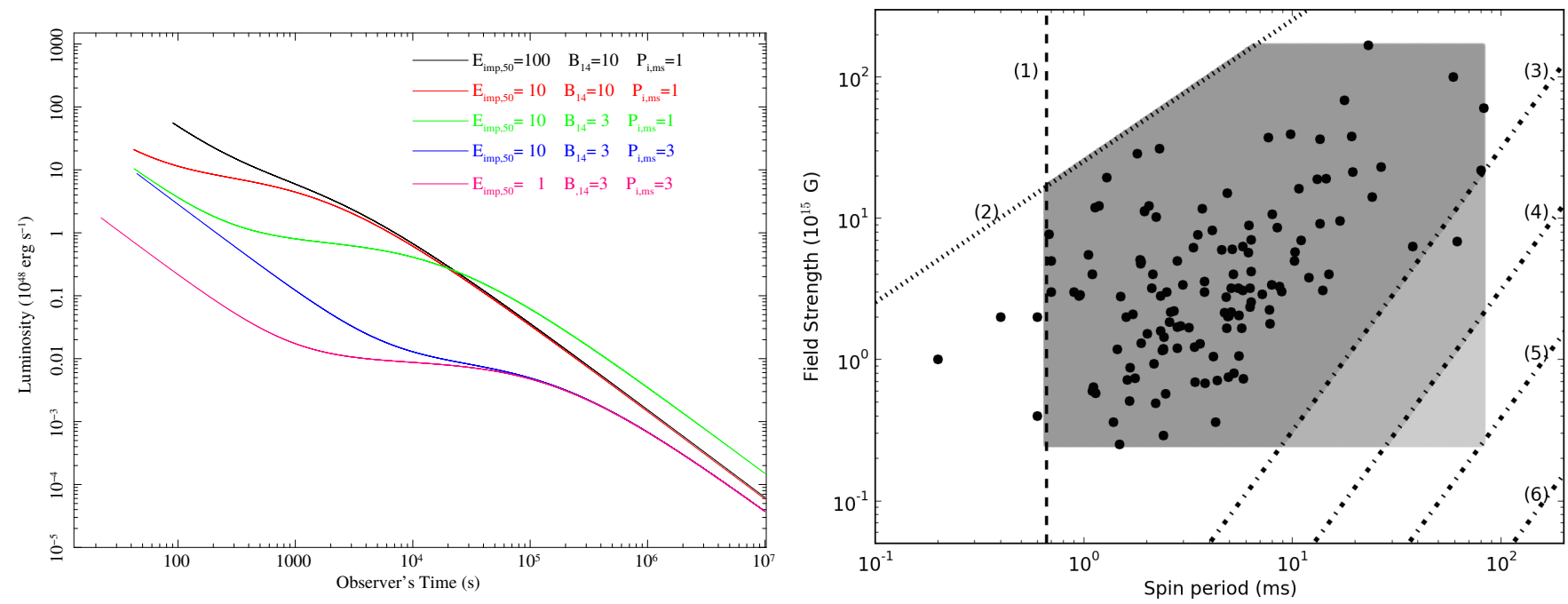

Figure 5. Left panel: five theoretical light curves obtained by Dall'Osso et al. (2011), changing the initial energy of the afterglow, the dipole magnetic field, B, and the initial spin period of the NS, P. Right panel: the grey shaded areas are the homogeneous distribution of $\mathrm{B}$ and $\mathrm{P}$ employed to simulate the observable magnetar plateaus from Rowlinson et al. (2014). The upper and lower limits on B and the upper limit on P are computed considering the sample of GRBs fitted with the magnetar model (Lyons et al. 2010; Dall'Osso et al. 2011; Bernardini et al. 2012a; Gompertz et al. 2013; Rowlinson et al. 2013; Yi et al. 2014; Lü and Zhang 2014). The dashed black vertical line (1) at $0.66 \mathrm{~ms}$ is the minimum $\mathrm{P}$ allowed. The dotted black line (2) indicates a limit on $\mathrm{P}$ and $\mathrm{B}$ strengths imposed by the fastest slew time of XRT in their sample in the rest frame of the highest $z$ GRB, as plateaus with durations shorter than the slew time are unobservable. The black dash-dot lines (3-6) are the observational constraints for the dimmest XRT plateau observable assuming the lowest $z$ in the GRB sample. These limits vary as a function of the beaming and efficiency of the magnetar emission: (3) Minimum beaming angle and efficiency (1 degree and 1\% respectively), (4) Minimum efficiency (1\%) and maximum beaming angle (isotropic), (5) Maximum efficiency (100\%) and minimum beaming angle, (6) Maximum efficiency and beaming angle. The observed distributions indicate that the samples have low efficiencies and small beaming angles.

Later, Rowlinson et al. (2014), using 159 GRBs from Swift catalogue, analytically demonstrated that the central engine model accounts for the LT relation assuming that the compact object is injecting energy into the forward shock (FS), a shock driven out into the surrounding circumstellar medium. The luminosity and plateau duration can be computed as follows:

$$
\log L_{X, a} \sim \log \left(B_{p}^{2} P_{0}^{-4} R^{6}\right)
$$

and

$$
\log T_{X, a}^{*}=\log \left(2.05 \times I B_{p}^{-2} P_{0}^{2} R^{-6}\right),
$$

where $T_{X, a}^{*}$ is in units of $10^{3} \mathrm{~s}, L_{X, a}$ is in units of $10^{49} \mathrm{erg} \mathrm{s}^{-1}, I$ is the moment of inertia in units of $10^{45} \mathrm{~g} \mathrm{~cm}^{2}$, $B_{p}$ is the magnetic field strength at the poles in units of $10^{15} \mathrm{G}, R$ is the radius of the NS in units of $10^{6} \mathrm{~cm}$ and $P_{0}$ is the initial period of the compact object in milliseconds. Then, substituting the radius from eq. 4 into eq. 3 , it was derived that:

$$
\log \left(L_{X, a}\right) \sim \log \left(10^{52} I^{-1} P_{0}^{-2}\right)-\log \left(T_{X, a}^{*}\right) .
$$

Therefore, an intrinsic relation $\log L_{X, a} \sim-\log T_{X, a}^{*}$ is confirmed directly from this formulation. Although some magnetar plateaus are inconsistent with energy injection into the FS, Rowlinson et al. (2014) showed that this emission is narrowly beamed and has $\leq 20 \%$ efficiency in conversion of rotational energy from the compact object into the observed plateau luminosity. In addition, the intrinsic LT relation slope, namely the one where the selection biases are appropriately removed, is explained within the spin-down of a newly formed magnetar at $1 \sigma$ level, see right panel of Fig. 5. The scatter in the relation is mainly due to the range of the initial spin periods.

After several papers discussing the origin of the LT relation within the context of the magnetar model, very recently a debate has been opened by Rea et al. (2015) on the reliability of this model as the correct interpretation for the X-ray plateaus. Using GRBs with known $z$ detected by Swift from its launch to August 2014, Rea et al. (2015) concluded that the initial magnetic field distribution, used to interpret the GRB X-ray plateaus within the magnetar model does 
not match the features of GRB-magnetars with the Galactic magnetar population. Therefore, even though there are large uncertainties in these estimates due to GRB rates, metallicity and star formation, the GRB-magnetar model in its present form is safe only if two kinds of magnetar progenitors are allowed. Namely, the GRB should be different from Galactic magnetar ones (for example for different metallicities) and should be considered supermagnetars (magnetars with an initial magnetic field significantly large). Finally, they set a limit of about $\leq 16$ on the number of stable magnetars produced in the Milky Way via a GRB in the past Myr. However, it can be argued that since the rates of Galactic magnetars and GRBs are really different, the number of Galactic magnetars cannot fully describe the origin of GRBs. In fact the Galactic magnetar rate is likely to be greater than $10 \%$ than the core collapse SNe rate, while GRB rate is much lower than that. In addition, the number of magnetars in the Milky Way may not be used as a constraint on the GRB rate because the spin-down rates of GRB magnetars should be very rapid. Due to the low GRB rate it would not be easy to detect these supermagnetars. Thus, it can be claimed that no conflict stands among this paper and the previous ones.
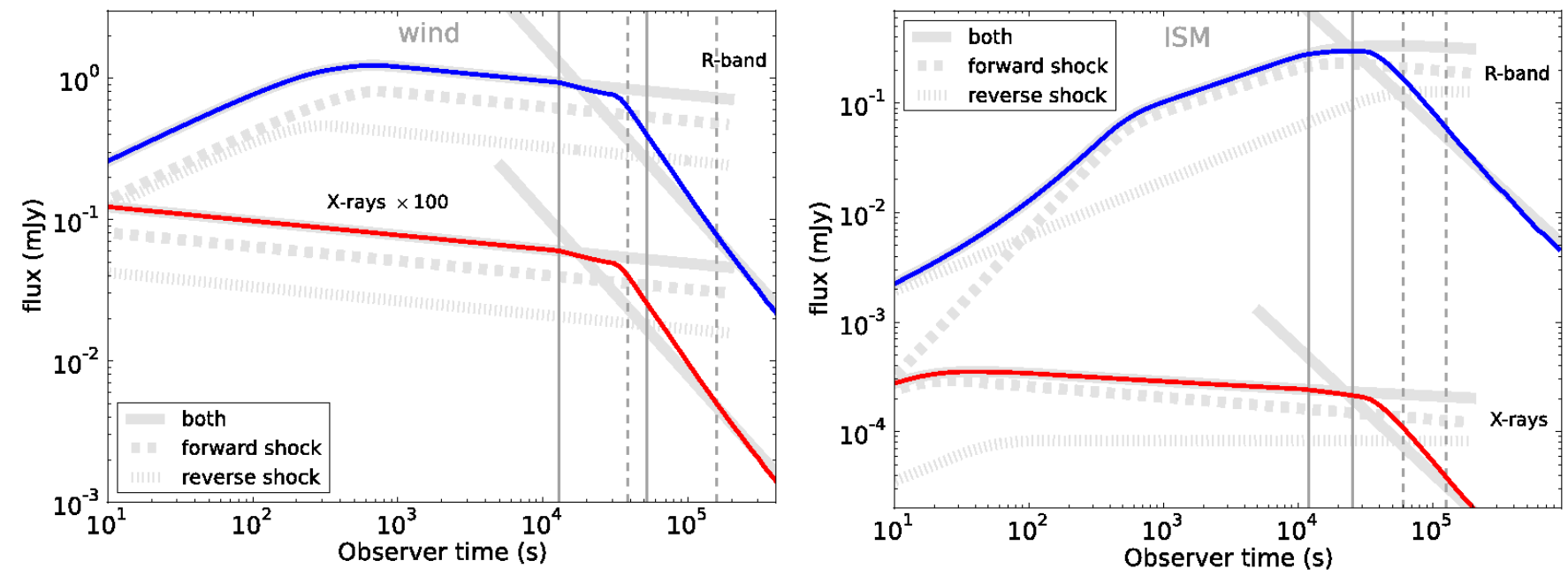

Figure 6. Optical and X-ray light curves for wind (left panel) and ISM (right plot) scenario's from van Eerten (2014a). Thick light grey curves represent the analytical solutions for prolonged and impulsive energy injection. Thick dashed light grey and the thick dotted light grey curves indicate the forward shock region emission only and the reverse shock region only respectively. The grey vertical lines show (1) the arrival time of emission from the jet back and (2) the arrival time of emission from the jet front. The solid vertical lines indicate arrival times of emission along the jet axis for these two events; the dashed vertical lines express the arrival times of emission from an angle $\theta=1 / \gamma$.

Still in the context of the energy injection models, van Eerten (2014a) found a relation between the optical flux at the end of the plateau and the time at the end of the plateau itself $F_{O, a} \sim T_{O, a}^{-0.78 \pm 0.08}$ (Panaitescu and Vestrand 2011; Li et al. 2012) for which observed frame variables were considered. The range of all parameters describing the emission $\left(E_{\gamma, i s o}\right.$, the fraction of the magnetic energy, $\epsilon_{B}$, the initial density, $\left.n_{0}\right)$ is the principal cause of the scatter in the relation, but it does not affect the slope. Finally, it was claimed that both the wind $\left(\propto A / r^{2}\right.$, where $\mathrm{A}$ is a constant) and the interstellar medium can reproduce the observed relation within both the reverse shock (RS, a shock driven back into the expanding bubble of the ejecta) and FS scenarios, see Fig. 6.

Considering alternative models explaining the LT relation, Sultana et al. (2013) studied the evolution of the Lorentz gamma factor, $\Gamma=1 / \sqrt{1-v^{2} / c^{2}}$ (where $v$ is the relative velocity between the inertial reference frames and $c$ is the light speed), during the whole duration of the light curves within the context of the Supercritical Pile Model. This model provides an explanation for both the steep-decline and the plateau or the steep-decline and the power law decay phase of the GRB afterglow, as observed in a large number of light curves, and for the LT relation. One of their most important results is that the duration of the plateau in the evolution of $\Gamma$ becomes shorter with a decreasing value of $M_{0} c^{2}$, where $M_{0}$ is the initial rest mass of the flow. This occurrence means that the more luminous the plateau, the shorter its duration and the smaller the $M_{0} c^{2}$, namely the energy.

Instead, in the context of the RS and FS emissions, Leventis et al. (2014), investigating the synchrotron radiation in the thick shell scenario (i.e. when the RS is relativistic), found that this radiation is compatible with the presence of the plateau phase, see the left panel of Fig. 7 . In addition, analyzing the $\log F_{X, a}-\log T_{X, a}$ relation in the framework of this model, they arrived at the conclusion that smooth energy injection through the RS is favoured respect to the FS, see the right panel of Fig. 7 . 

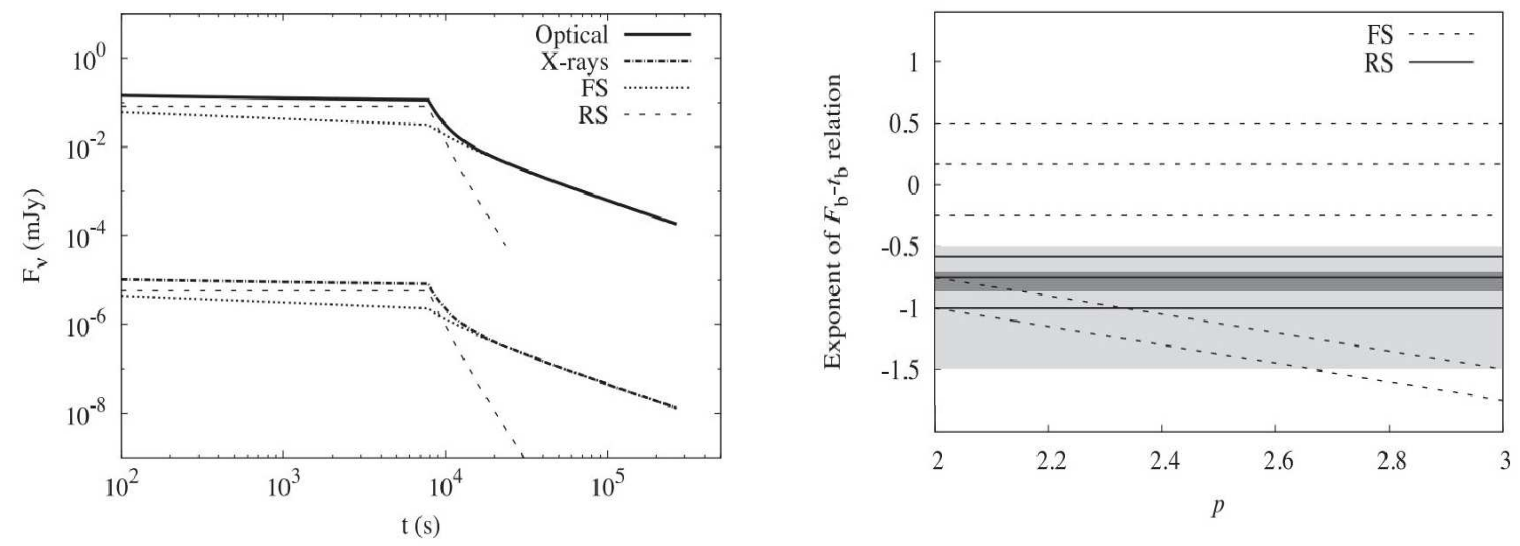

Figure 7. Left panel: optical and X-ray light curves before and after the injection break from Leventis et al. (2014). The contributions of the FS (dotted line) and RS (dashed line) are shown for both. The considered parameters are $E=10^{51} \mathrm{erg}, n_{1}=50 \mathrm{~cm}-3, \Delta t=5 \times 10^{3}$ $\mathrm{s}, \eta=600, \mathrm{q}=0, \epsilon_{e}=\epsilon_{B}=0.1, \mathrm{p}=2.3, \theta_{j}=90^{\circ}, d=10^{28} \mathrm{~cm}$ and $z=0.56$. Right panel: index of the $F_{X, a}-T_{X, a}$ relation as a function of the electron distribution index, p, for the FS and the RS from Leventis et al. (2014). The lightly shaded region includes values allowed by the scaling from Panaitescu and Vestrand (2011), while the darker region indicates the scaling from Li et al. (2012). The five dashed lines show the five possible indices for the FS, while the three solid lines display the three possible (independent of p) indices for the RS.
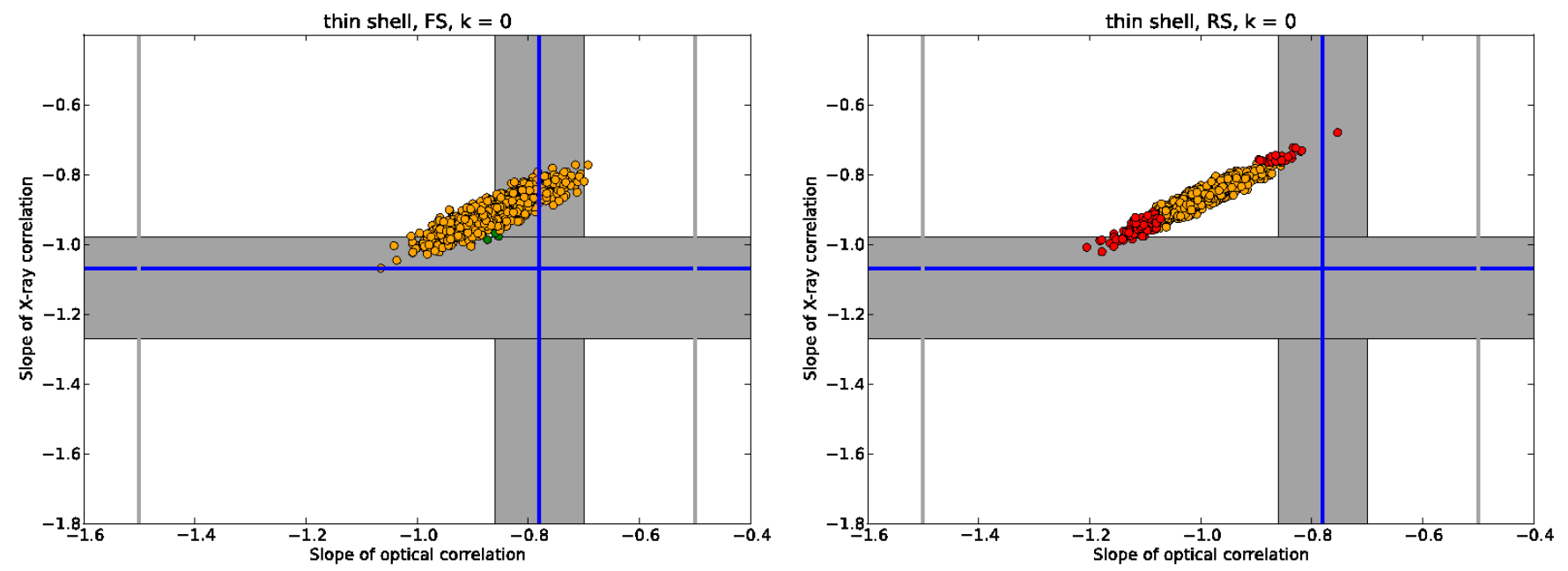

Figure 8. Comparison of the slopes for 1000 thin shell data set runs and slopes of the observed LT relation in optical (horizontal direction) and the LT relation in X-ray (vertical direction) from van Eerten (2014b) for the FS (left panel) and the RS (right panel) cases. Grey band expresses $1 \sigma$ errors on the relations, while green dots represent runs consistent at $1 \sigma$ error bars for both, orange dots are compatible at $3 \sigma$, but not at $1 \sigma$ and red dots pass neither test. Vertical grey lines show more scattered LT in optical error bars from Panaitescu and Vestrand (2011).

van Eerten (2014b), with a simulated sample of GRBs, found out that the observed LT relation rules out basic thin shell models, but not basic thick ones. In fact, in the thick model, the plateau phase comes from the late central source activity or from additional kinetic energy transfer from slower ejecta which catches up with the blast wave. As a drawback, in this context, it is difficult to distinguish between FS and RS emissions, or homogeneous and stellar wind-type environments.

In the thin shell case, the plateau phase is given by the pre-deceleration emission from a slower component in a twocomponent or jet-type model, but this scenario is not in agreement with the observed LT relation, see Fig. 8. This, however, does not imply that acceptable fits using a thin shell model are not possible, but further analysis is needed to exclude without any doubts thin shell models. Another model which has not been tested yet on this correlation is the photospheric emission model from stratified jets (Ito et al. 2014). 


\subsection{The unified $L_{X}\left(T_{a}\right)-T_{X, a}^{*}$ and $L_{O, a}-T_{O, a}^{*}$ relations}

In order to describe the unified picture of the X-ray and optical afterglow, it is necessary to introduce relevant features regarding optical luminosities. To this end, Boër and Gendre (2000) studied the afterglow decay index in 8 GRBs in both X-ray and optical wavelengths. In the X-ray, the brightest GRBs had decay indices around 1.6 and the dimmest GRBs had decay indices around 1.11. Instead, they didn't observe this trend for the optical light curves, probably due to the host galaxy absorption.

Later, Nardini et al. (2006) discovered that the monochromatic optical luminosities at 12 hours, $L_{O, 12}$, of 24 LGRBs cluster at $\log L_{O, 12}=30.65 \mathrm{erg} \mathrm{s}^{-1} \mathrm{~Hz}^{-1}$, with $\sigma_{\text {int }}=0.28$. The distribution of $L_{O, 12}$ is less scattered than the one of $L_{X, 12}$, the luminosity at 12 hours in the X-ray, and the one of the ratio $L_{O, 12} / E_{\gamma, \text { prompt }}$, where $E_{\gamma, \text { prompt }}$ is the rest frame isotropic prompt energy. Three bursts are outliers because they have luminosity which is smaller by a factor $\sim 15$. This result suggests the existence of a family of intrinsically optically underluminous dark GRBs, namely GRBs where the optical-to-X-ray spectral index, $\beta_{O X, a}$, is shallower than the X-ray spectral index minus $0.5, \beta_{X, a}-0.5$.

Liang and Zhang (2006a) confirmed these results. They found a bimodal distribution of $L_{O, 1 \mathrm{~d}}$ using 44 GRBs. Nardini et al. (2008a) also confirmed these findings. They analyzed selection effects present in their observations extending the sample to $55 \mathrm{LGRBs}$ with known $z$ and rest-frame optical extinction detected by the Swift satellite.

In contrast, Melandri et al. (2008), Oates et al. (2009), Zaninoni et al. (2013) and Melandri et al. (2014) found no bimodality in the distributions of $L_{O, 12}, L_{O, 1 \mathrm{~d}}$ and $L_{O, 11}$, investigating samples of $44,24,40$ and 47 GRBs respectively. Instead, with the aim of finding a unifying representation of the GRB afterglow phase, Ghisellini et al. (2009) fitted the light curves assuming this functional form:

$$
L_{L}(\nu, t)=L_{L}\left(\nu, T_{X, a}\right) \frac{\left(t / T_{X, t}\right)^{-\alpha_{\nu, f l}}}{1+\left(t / T_{X, t}\right)^{\alpha_{\nu, s t}-\alpha_{\nu, f l}}} .
$$

They used a data sample of 33 LGRBs detected by Swift in X-ray (0.3-10 keV) and optical R bands (see the left and middle panels of Fig. 9). Within this approximation, the agreement with data is reasonably good, and they confirmed the X-ray LT relation.
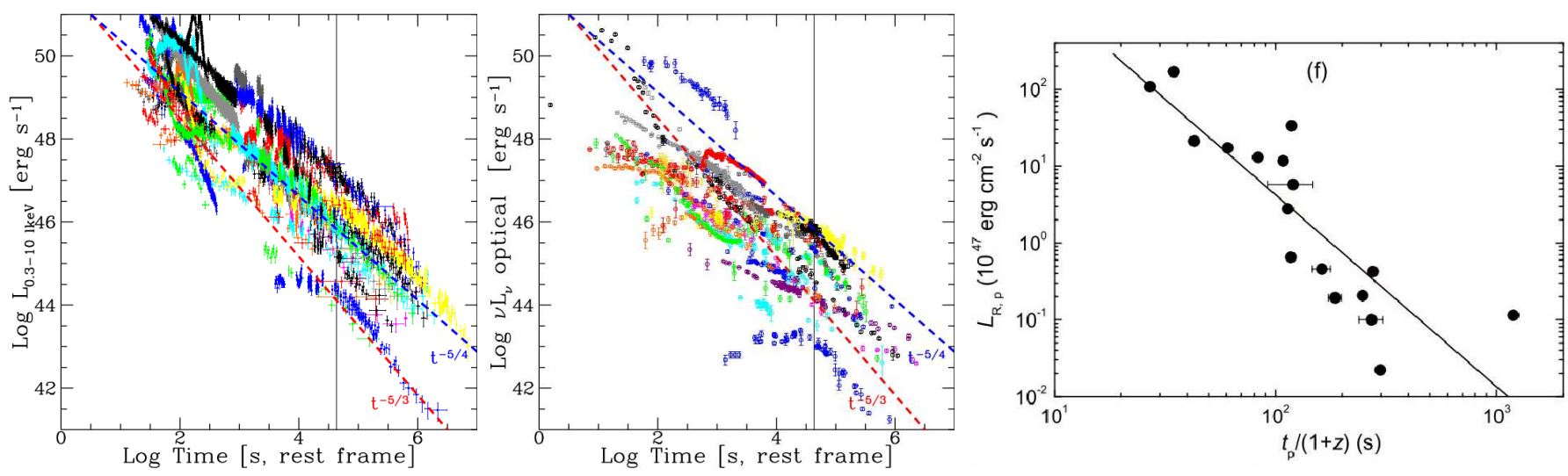

Figure 9. The light curves of the full sample from Ghisellini et al. (2009) in the X-rays (left panel) and optical (middle panel). The vertical lines represent $\log L_{X, 12}$ and $\log L_{O, 12}$ in the rest frame time respectively. Instead, the dashed lines indicate the log $t^{-5 / 4}$ (blue) and the $\log t^{-5 / 3}$ (red) behaviours. Right panel: relation between $L_{O, \text { peak }}$ (equivalent to $L_{R, p}$ in the picture) and $T_{O, p e a k}^{*}$ of the data set from Liang et al. (2010). Line represents the best fit.

Through their analysis using a data sample of 32 Swift GRBs, Liang et al. (2010) found that the optical peak luminosity, $L_{O, p e a k}$, in the $\mathrm{R}$ band in units of $10^{47} \mathrm{erg} \mathrm{s}^{-1}$ and the optical peak time, $T_{O \text {,peak }}^{*}$, are anti-correlated, see the right panel of Fig. 9, with a slope $b=-2.49 \pm 0.39$ and $\rho=-0.90$. They deduced that a fainter bump has its maximum later than brighter ones and it also presents a longer duration.

Panaitescu and Vestrand (2011) showed a similar relation to the one presented in Liang et al. (2010). They found a $\log F_{O, a} \sim \log T_{O, a}^{-1}$ anti-relation using 37 Swift GRBs. This result may indicate a unique mechanism for the optical afterglow even though the optical energy has a quite large scatter.

Afterwards, Li et al. (2012) found a relation (see the left panel of Fig. 10) similar to the LT relation, but in the R band. They used 39 GRBs with optical data available in the literature. This relation is between the optical luminosity at the end of the plateau, $L_{O, a}$, in units of $10^{48} \mathrm{erg} \mathrm{s}^{-1}$ and the optical end of the plateau time, $\log T_{O, a}^{*}$, in the shallow 

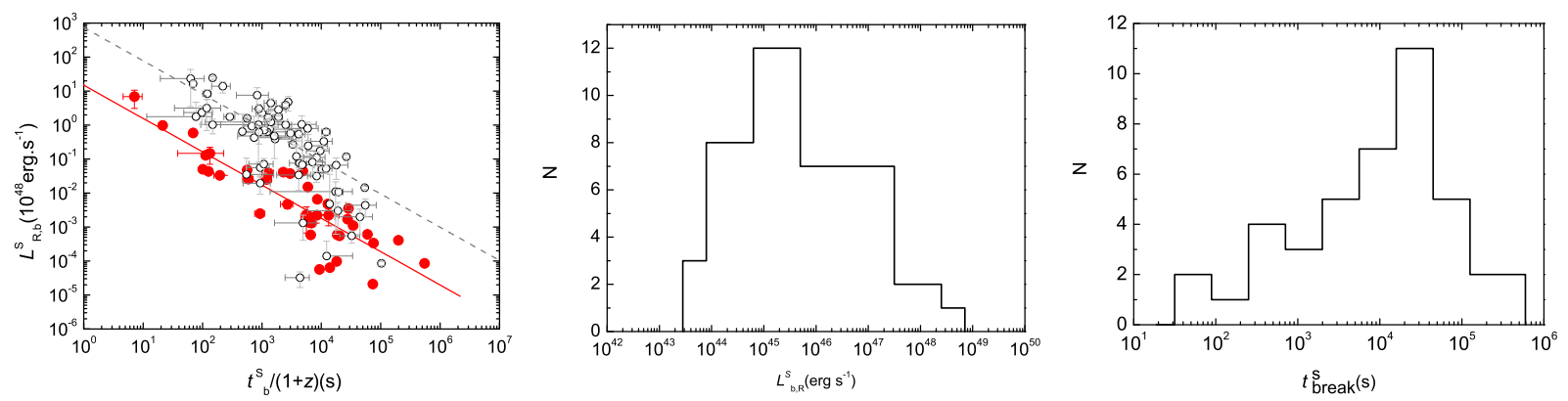

Figure 10. Left panel: $L_{O, a}^{S}$ (equivalent to $L_{R, p}^{S}$ in the picture) as a function of $T_{O, a}^{S, *}$ (equivalent to $t_{b}$ in the picture) from $L i$ et al. (2012). The grey circles represent the X-ray data from Dainotti et al. (2010). Lines correspond to the best fit lines. Middle and Right panels: $L_{O, a}^{S}$ and $T_{O, a}^{S}$ distributions for the full GRB data set from Li et al. (2012).

decay phase of the GRB light curves, denoted with the index S. They found a slope $b=-0.78 \pm 0.08, \rho=0.86$ and $P<10^{-4}$.

\begin{tabular}{|c|c|c|c|c|c|}
\hline Correlations & Author & $\mathrm{N}$ & Slope & Corr.coeff. & $\mathrm{P}$ \\
\hline$L_{O, \text { peak }}-T_{O, \text { peak }}$ & Liang et al. (2010) & 32 & $-2.49 \pm 0.39$ & -0.90 & \\
$L_{O, a}-T_{O, a}^{S}$ & Panaitescu \& Vestrand (2011) & 37 & -1 & & \\
$L_{O, a}^{S}-T_{O, a}^{S}$ & Li et al. (2012) & 39 & $-0.78 \pm 0.08$ & 0.86 & $<10^{-4}$ \\
\hline
\end{tabular}

Table 3. Summary of the relations in this section. The first column represents the relation in log scale, the second one the authors, and the third one the number of GRBs in the used sample. Afterwards, the fourth column is the slope of the relation and the last two columns are the correlation coefficient and the chance probability, P.

$L_{O, a}^{S}$ varies from $10^{43}$ to $10^{47} \mathrm{erg} \mathrm{s}^{-1}$, and in some GRBs with an early break reaches $\sim 10^{49} \mathrm{erg} \mathrm{s}^{-1}$, see the middle panel of Fig. 10. $T_{O, a}^{S}$ spans from tens of seconds to several days after the GRB trigger, with a typical shallow peak time $T_{O, a}^{S}$ of $\sim 10^{4}$ seconds, see the right panel of Fig. 10. By plotting $L_{O, a}$ in units of $10^{48} \mathrm{erg} \mathrm{s}^{-1}$ as a function of $T_{O, a}^{*}$ in the burst frame, they observed that optical data have a similar trend to the X-ray data. In fact, this power law relation, presented in the left panel of Fig. 10, with an index of $-0.78 \pm 0.08$ is similar to that derived for the X-ray flares (see sec. 4.6). XRF phenomena are described in sec. 1. As a consequence, they recovered the LT relation. In Table 3 a summary of the relations described in this section is displayed.

\subsubsection{Physical interpretation of the unified $L_{X}\left(T_{a}\right)-T_{X, a}^{*}$ and $L_{O, a}-T_{O, a}^{*}$ relations}

In the unified $L_{X}\left(T_{a}\right)-T_{X, a}^{*}$ and $L_{O, a}-T_{O, a}^{*}$ relations Ghisellini et al. (2009) considered the flux as the sum of synchrotron radiation caused by the standard FS due to the fireball impacting the circumburst medium and of another component may be produced by a long-lived central engine, which resembles mechanisms attributed to a "late prompt". Even if based in part on a phenomenological model, Ghisellini et al. (2009) explained situations in which achromatic and chromatic jet break are either present or not in the observed light curves.

In addition, from their analysis, the decay slope of the late prompt emission results to be $\alpha_{X, a}=-5 / 4$ (see blue dashed line for X-ray and optical emission in the left and middle panels of Fig. 9 respectively), really close within the errors to the value of the temporal accretion rate of fall-back material (i.e. $\sim \log t^{-5 / 3}$, see red dashed line for X-ray and for optical emission in the left and middle panels of Fig. 9 respectively). This explains the activity of the central engine for such a long duration. For a similar interpretation within the context of the accretion onto the $\mathrm{BH}$ related to LT relation see sec. 3.1.1.

Liang et al. (2010) claimed that the external shock model explains well the anti-relation between $L_{O, p e a k}$ and $T_{O, p e a k}$, because later deceleration time is equivalent to slower ejecta and thus to a less luminous emission.

Furthermore, Panaitescu and Vestrand (2008) from the analysis of the $\log L_{O, a}-\log T_{O, a}^{*}$ relation explained the peaky afterglows (those with $L_{O, a} \propto T_{O, a}^{-1}$ ) as being a bit outside the cone of view, while the plateau as off-axis events and due to the angular structure of the jet. Later, Panaitescu and Vestrand (2011) asserted that the double-jet structure of the ejecta is problematic. To overcome this issue, they suggested a model in which both the peaky and plateau afterglows depend on how much time the central engine allows for the energy injection. More specifically, impulsive ejecta with a narrow range of $\Gamma$ are responsible for the peaky afterglows, while the plateau afterglows are produced by a distribution of initial $\Gamma$ which keeps the energy injection till $10^{5} \mathrm{~s}$. 
Later, Li et al. (2012) pointed out that late GRB central engine activities can account for both optical flares and the optical shallow-decay segments. These activities can be either erratic (for flares) or steady (for internal plateaus). A normal decay follows the external plateaus with $\alpha_{X, a}$ typically around -1 , thus possibly originated by an external shock with the shallow decay segment caused by continuous energy injection into the blast wave (Rees and Mészáros 1998; Dai and Lu 1998; Sari and Mészáros 2000; Zhang and Mészáros 2001). Instead, the internal plateaus, found first by Troja et al. (2007) in GRB 070110 and later studied statistically by Liang et al. (2007), are followed by a much steeper decay $\left(\alpha_{X, a}\right.$ steeper than -3$)$, which needs to be powered by internal dissipation of a late outflow. In summary, the afterglow can be interpreted as a mix of internal and external components.

\subsection{The $L_{O, 200 \mathrm{~s}}-\alpha_{O,>200 \mathrm{~s}}$ relation and its physical interpretation}

Oates et al. (2012) discovered a relation between the optical luminosity at $200 \mathrm{~s}, \log L_{O, 200 \mathrm{~s}}$, and the optical temporal decay index after $200 \mathrm{~s}, \alpha_{O,>200 \mathrm{~s}}$, see the right panel of Fig. 11. They used a sample of $48 \mathrm{LGRB}$ afterglow light curves at $1600 \AA$ detected by UVOT on board of the Swift satellite, see the left panel of Fig. 11. The best fit line for this relation is given by:

$$
\log L_{O, 200 \mathrm{~s}}=(28.08 \pm 0.13)-(3.636 \pm 0.004) \times \alpha_{O,>200 \mathrm{~s}},
$$

with $\rho=-0.58$ and a significance of $99.998 \%(4.2 \sigma)$. This relation means that the brightest GRBs decay faster than the dimmest ones. To obtain the light curves employed for building the relation, they used the criteria from Oates et al. (2009) in order to guarantee that the entire UVOT light curve is not noisy, namely with a high signal to noise $(\mathrm{S} / \mathrm{N})$ ratio: the optical/UV light curves must be observed in the $\mathrm{V}$ filter of the UVOT with a magnitude $\leq 17.8$, UVOT observations must have begun within the first $400 \mathrm{~s}$ after the BAT trigger and the afterglow must have been observed until at least $10^{5} \mathrm{~s}$ after the trigger. Their results pointed out the dependence of this relation is on the differences in the observing angle and on the rate of the energy release from the central engine.

As a further step, Oates et al. (2015), using the same data set, investigated the same relation both in optical and in X-ray wavelengths in order to make a comparison, and they confirmed previous optical results finding a similar slope for both relations. In addition, they analyzed the connection between the temporal decay indices after $200 \mathrm{~s}$ (in X-ray and optical) obtaining as best fit relation $\alpha_{X,>200 \mathrm{~s}}=\alpha_{O,>200 \mathrm{~s}}-0.25$, see the left panel of Fig. 12. They yielded some similarities between optical and X-ray components of GRBs from these studies. Their results were in disagreement with those previously found by Urata et al. (2007), who investigated the relation between the optical and X-ray temporal decay indices in the normal decay phase derived from the external shock model. In fact, a good fraction of outliers was found in this previous work.

Racusin et al. (2016) studied a similar relation using 237 Swift LGRBs, but in X-ray. For this relation, it was found that slope $b=-0.27 \pm 0.04$ and solid evidence for a strong connection between optical and X-ray components of GRBs was discovered as well. In conclusion, the Monte Carlo simulations and the statistical tests validated the relation between $\log L_{O, 200 \text { s }}$ and $\alpha_{O,>200 \text { s }}$ by Oates et al. (2012). In addition, it shows a possible connection with its equivalent, the LT relation in X-ray, implying a common physical mechanism. In Table 4 a summary of the relations described in this section is reported.

Regarding the physical interpretation of the $\log L_{O, 200 \mathrm{~s}}-\alpha_{O,>200 \mathrm{~s}}$ relation, Oates et al. (2012) explored several scenarios. The first one implies that the relation can be due to the interaction of the jet with the external medium. In a straightforward scenario $\alpha_{O,>200 \mathrm{~s}}$ is not a fixed value and all optical afterglows stem from only one closure relation where $\alpha_{O,>200 \mathrm{~s}}$ and $\beta_{O,>200 \mathrm{~s}}$ are related linearly. Thus a relation between $\log L_{O, 200 \mathrm{~s}}$ and $\beta_{O,>200 \mathrm{~s}}$ should naturally appear. Contrary to this expectation, $\alpha_{O,>200 \mathrm{~s}}$ and $\beta_{O,>200 \mathrm{~s}}$ are poorly correlated, see the right panel of Fig. 12, and there is no evidence for the existence of a relation between $\beta_{O,>200 \mathrm{~s}}$ and $\log L_{O, 200 \mathrm{~s}}$. Therefore, this scenario cannot be ascribed as the cause of the $\log L_{O, 200 \mathrm{~s}}-\alpha_{O,>200 \mathrm{~s}}$ relation.

In the second scenario, they assumed that the $\log L_{O, 200 \mathrm{~s}}-\alpha_{O,>200 \mathrm{~s}}$ relation is produced by few closure relations indicated by lines in the right panel of Fig. 12. However, from this picture, the $\alpha_{O,>200 s}$ and $\beta_{O,>200 s}$ values with similar luminosities do not gather around a particular closure relation, thus also the basic standard model is not a good explanation of the $\log L_{O, 200 \mathrm{~s}}-\alpha_{O,>200 \mathrm{~s}}$ relation. As a conclusion, the afterglow model is more complex than it was considered in the past. It is highly likely that there are physical properties that control the emission mechanism and the decay rate in the afterglow that still need to be investigated.

Therefore, Oates et al. (2012) proposed two additional alternatives. The first is related to some properties of the central engine influencing the rate of energy release so that for fainter afterglows, the energy is released more slowly. Otherwise, the relation can be due to different observing angles where observers at smaller viewing angles see brighter 

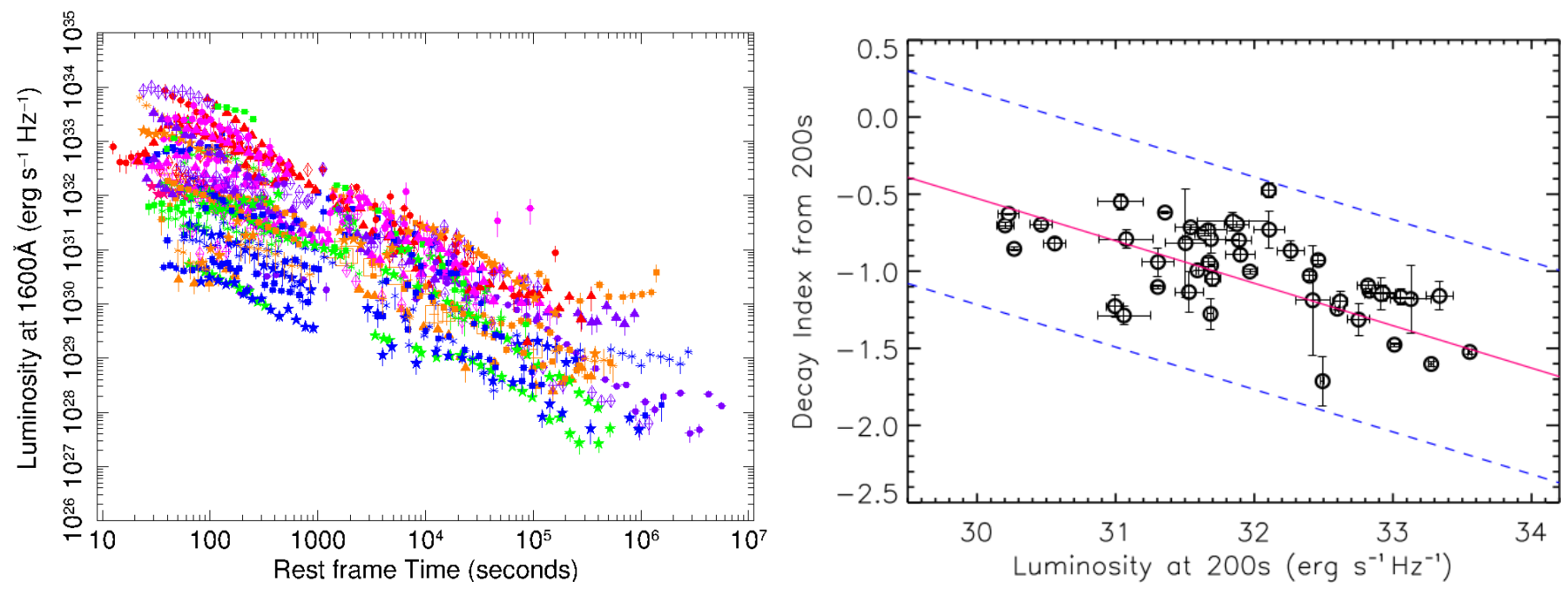

Figure 11. Left panel: "optical light curves of 56 GRBs from Oates et al. (2012)". Right panel: "log $L_{O, 200 s}$ vs. $\alpha_{O,>200 s}$ from Oates et al. (2012). The red solid line indicates the best fit line and the blue dashed lines show the $3 \sigma$ variance".
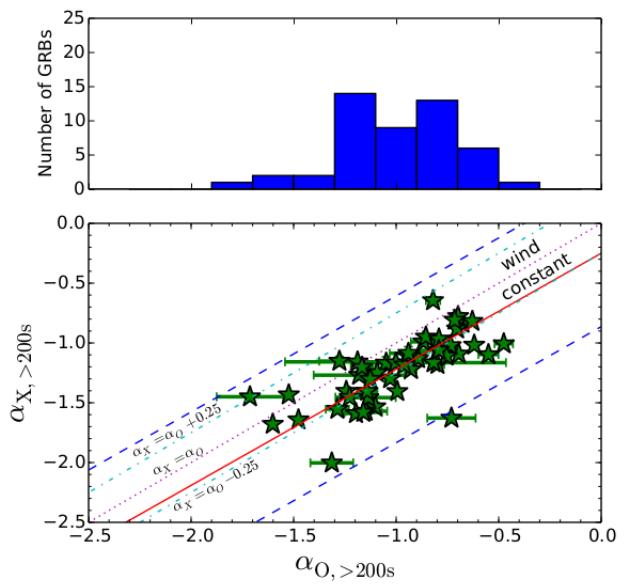

$\rho=0.77$

$\mathrm{P}=1.10 \mathrm{e}-10$

Slope $=0.97 \pm 0.10$

Const $=-0.25 \pm 0.09$

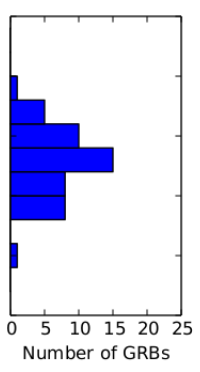

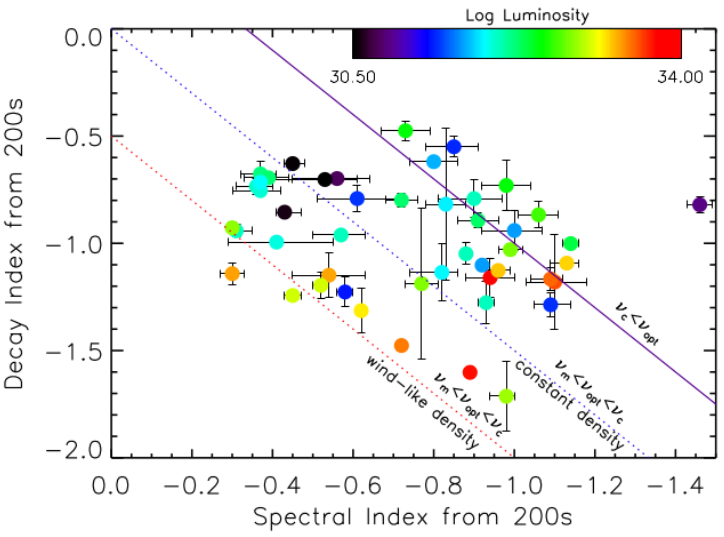

Figure 12. Left panel: " $\alpha_{O,>200 \mathrm{~s}}$ and $\alpha_{X},>200$ s from Oates et al. (2015). The red solid line represents the best fit regression and the blue dashed lines represent 3 times the root mean square (RMS) deviation. The relationships expected between the optical/UV X-ray light curves from the GRB closure relations are also shown. The pink dotted line represents $\alpha_{O}>200 \mathrm{~s}=\alpha_{X}>200 \mathrm{~s}$. The light blue dotted-dashed lines represent $\alpha_{X,>200 \mathrm{~s}}=\alpha_{O,>200 \mathrm{~s}} \pm 0.25$. In the top right corner it is given the coefficient $\rho$ with $P$, and it is provided the best fit slope and constant determined by linear regression". Right panel: " $\alpha_{O,>200 \text { s }}$ and $\beta_{O,>200 \text { s }}$ for the sample of 48 LGRBs from Oates et al. (2012). The lines represent 3 closure relations and a colour scale is used to display the range in $\log L_{O, 200 s "}$.

and faster decaying light curves.

\begin{tabular}{|c|c|c|c|c|c|c|}
\hline Correlations & Author & $\mathrm{N}$ & Slope & Norm & Corr.coeff. & $\mathrm{P}$ \\
\hline$L_{O, 200 \mathrm{~s}}-\alpha_{O,>200 \mathrm{~s}}$ & Oates et al. (2012) & 48 & $-3.636 \pm 0.004$ & $28.08 \pm 0.13$ & -0.58 & $2 \times 10^{-4}$ \\
& Oates et al. (2015) & 48 & $-3.636 \pm 0.004$ & $28.08 \pm 0.13$ & -0.58 & $2 \times 10^{-4}$ \\
$L_{X, 200 \mathrm{~s}}-\alpha_{X,>200 \mathrm{~s}}$ & Racusin et al. (2016) & 237 & $-0.27 \pm 0.04$ & $-6.99 \pm 1.11$ & 0.59 & $10^{-6}$ \\
\hline
\end{tabular}

Table 4. Summary of the relations in this section. The first column represents the relation in log scale, the second one the authors, and the third one the number of GRBs in the used sample. Afterwards, the fourth and fifth columns are the slope and normalization of the relation and the last two columns are the correlation coefficient and the chance probability, P.

As pointed out by Dainotti et al. (2013a), the $\log L_{O, 200 \mathrm{~s}}-\alpha_{O,>200 \mathrm{~s}}$ relation is related to the LT one since both show an anti-relation between luminosity and decay rate of the light curve or time. The key point would be to understand how they relate to each other and the possible common physics that eventually drives both of them. To this end, Oates et al. (2015) compared the observed relations with the ones obtained with the simulated sample. The luminosity-decay 
relationship in the optical/UV is in agreement with that in the X-ray, inferring a common mechanism.

\section{THE PROMPT-AFTERGLOW RELATIONS}

As we have discussed in the previous paragraphs, the nature of the plateau and the relations (e.g. the optical one) based on similar physics and directly related to the plateau are still under investigation. For this reason, several models have been proposed. To further enhance its theoretical understanding, it is necessary to evaluate the connection between plateaus and prompt phases. To this end, we hereby review the prompt-afterglow relations, thus helping to establish a more complete picture of the plateau GRB phenomenon.

\subsection{The $E_{\gamma, \text { afterglow }}-E_{X, \text { prompt }}$ relation and its physical interpretation}

W07 analyzed the relation between the gamma flux in the prompt phase, $F_{\gamma, p r o m p t}$, and the X-ray flux in the afterglow, $F_{X, \text { afterglow }}$ using 107 Swift GRBs, see the upper left panel of Fig. 13. They calculated $F_{X, \text { afterglow }}$ in the XRT band (0.3-10 keV), while $F_{\gamma, p r o m p t}$ in the BAT (15-150 keV) plus the XRT (0.3-10 keV) energy band. For GRBs with known redshift, as shown in the upper right panel of Fig. 13, they investigated the prompt isotropic energy, $E_{\gamma, \text { prompt }}$, and the afterglow isotropic energy, $E_{X, \text { afterglow }}$, assuming a cosmology with $H_{0}=71 \mathrm{~km} \mathrm{~s}^{-1} \mathrm{Mpc}^{-1}$, $\Omega_{\Lambda}=0.73$ and $\Omega_{M}=0.27$.

At the same time, Liang et al. (2007) focused on the relation between $E_{\gamma, \text { prompt }}$ and $E_{X, \text { afterglow }}$ using a sample of 53 LGRBs. They pointed out a good relation with $b=1 \pm 0.16$, see the bottom left panel of Fig. 13.

In agreement with these results, Liang et al. (2010) and Panaitescu and Vestrand (2011) analyzed this relation, using respectively 32 and 37 GRBs, but considering energy bands different from that used in Liang et al. (2007); they obtained the slopes $b=0.76 \pm 0.14$ and $b=1.18$ respectively (see the left and middle panels of Fig. 14).

Rowlinson et al. (2013) and Grupe et al. (2013) confirmed these results, see the left and middle panels of Fig. 15.

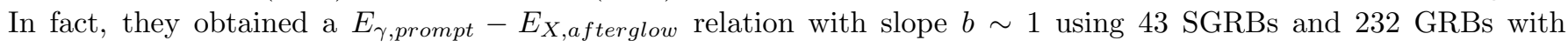
spectroscopic redshifts detected by Swift respectively.

Finally, Dainotti et al. (2015b) analyzed this relation to find some constraints on the ratio of $E_{X, \text { afterglow }}$ to $E_{\gamma, \text { prompt }}$, considering a sample of 123 LGRBs, see the right panel of Fig. 15.

Instead, Ghisellini et al. (2009), with a sample of 33 LGRBs, considered a similar relation, but assuming the X-ray plateau energy, $E_{X, \text { plateau }}$, as an estimation of $E_{X, \text { afterglow }}$, see the bottom right panel of Fig. 13; they found a slope $b=0.86$.

In addition, Ghisellini et al. (2009) also investigated the relation between $E_{\gamma, \text { prompt }}$ and the kinetic isotropic energy in the afterglow, $E_{k, a f t}$, with the same sample, finding a relation with $b=0.42$. Similarly, Racusin et al. (2011) studied the same relation, using $69 \mathrm{GRBs}$ and assuming different efficiencies to find some limits between $E_{k, \text { aft }}$ and $E_{\gamma, \text { prompt }}$, see the right panel of Fig. 14.

This relation was most likely used to study the differences in detection of several instruments and to analyze the transferring process of kinetic energy into the prompt emission in GRBs, making the relation by Racusin et al. (2011) the most reliable one.

To summarize, for comparing the energies in the prompt and the afterglow phases, a $E_{\gamma, \text { prompt }}-E_{X, \text { afterglow }}$ relation was studied by Liang et al. (2007) and confirmed by Rowlinson et al. (2013), Grupe et al. (2013) and Dainotti et al. (2015b). The last study found also some limitations on the ratio among the prompt and the afterglow energies. Furthermore, instead of $E_{X, \text { afterglow }}, E_{X, \text { plateau }}$ was considered for the investigation, although this quantity provided similar results to the previous ones (Ghisellini et al. 2009). Finally, the relation between $E_{\gamma, p r o m p t}$ and $E_{k, \text { aft }}$ was studied by Ghisellini et al. (2009) and confirmed by Racusin et al. (2011), who examined the energy transfer in the prompt phase. These relations are relevant because of their usefulness for investigating the efficiency of the emission processes during the transition from the prompt phase to the afterglow one, and for explaining which the connection between these two emission phases is. As a main result, Ghisellini et al. (2009) and Racusin et al. (2011) claimed that the fraction of kinetic energy transferred from the prompt phase to the afterglow one is around 10\%. In particular, Racusin et al. (2011) yielded that this value of the transferred kinetic energy, for BAT-detected GRBs, is in agreement with the analysis by Zhang et al. (2007a) for which the internal shock model well describes this value in the case of a late energy transfer from the fireball to the surrounding medium (Zhang and Kobayashi 2005).

In Table 5, a summary of the relations described in this section is presented.

As regards the physical interpretation of the $E_{X, \text { afterglow }}-E_{\gamma, \text { prompt }}$ relation, Racusin et al. (2011), estimating the efficiency parameter $\eta$ for the BAT sample, confirmed the Zhang et al. (2007a) result for which $\sim 57 \%$ of BAT bursts have $\eta<10 \%$. However, for the samples from the Gamma Burst Monitor (GBM) and the Large Area Telescope 

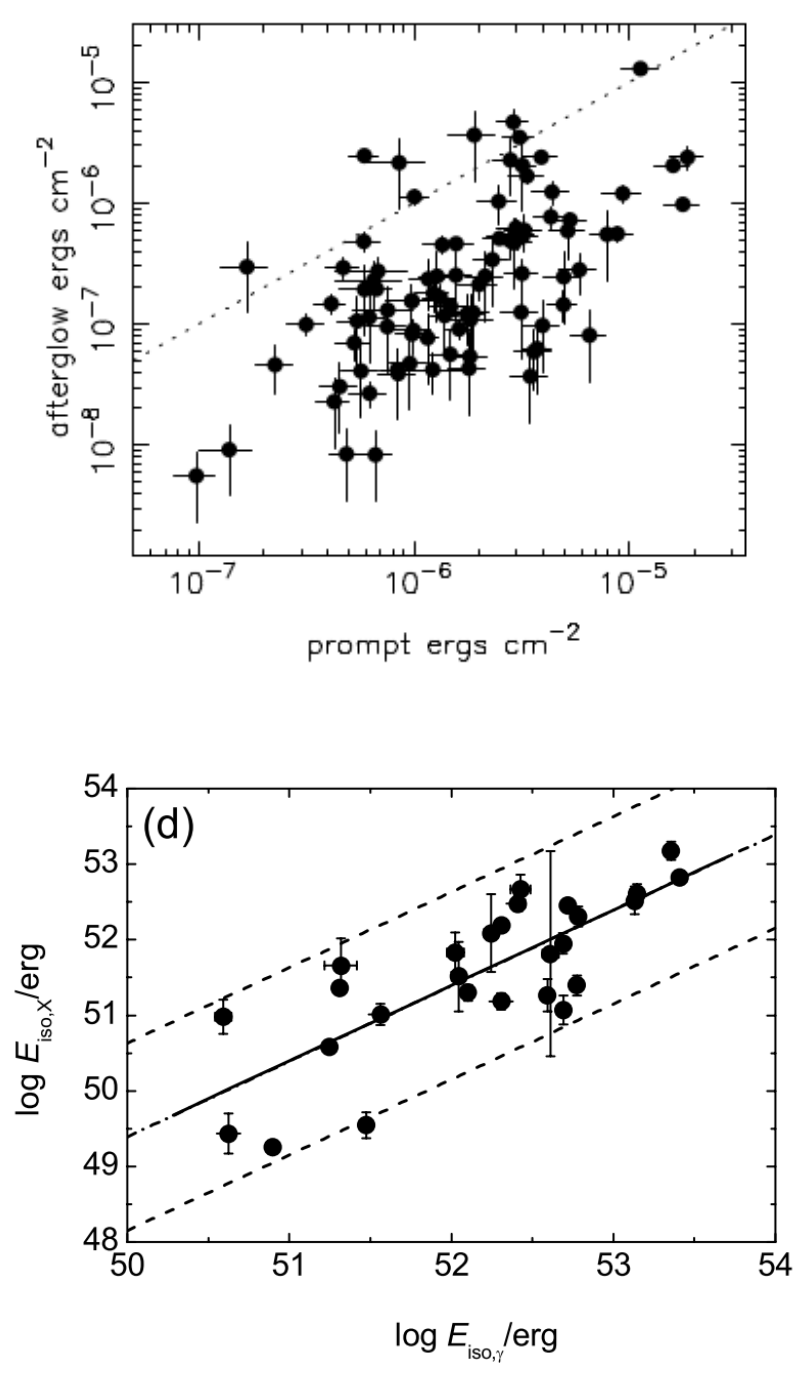
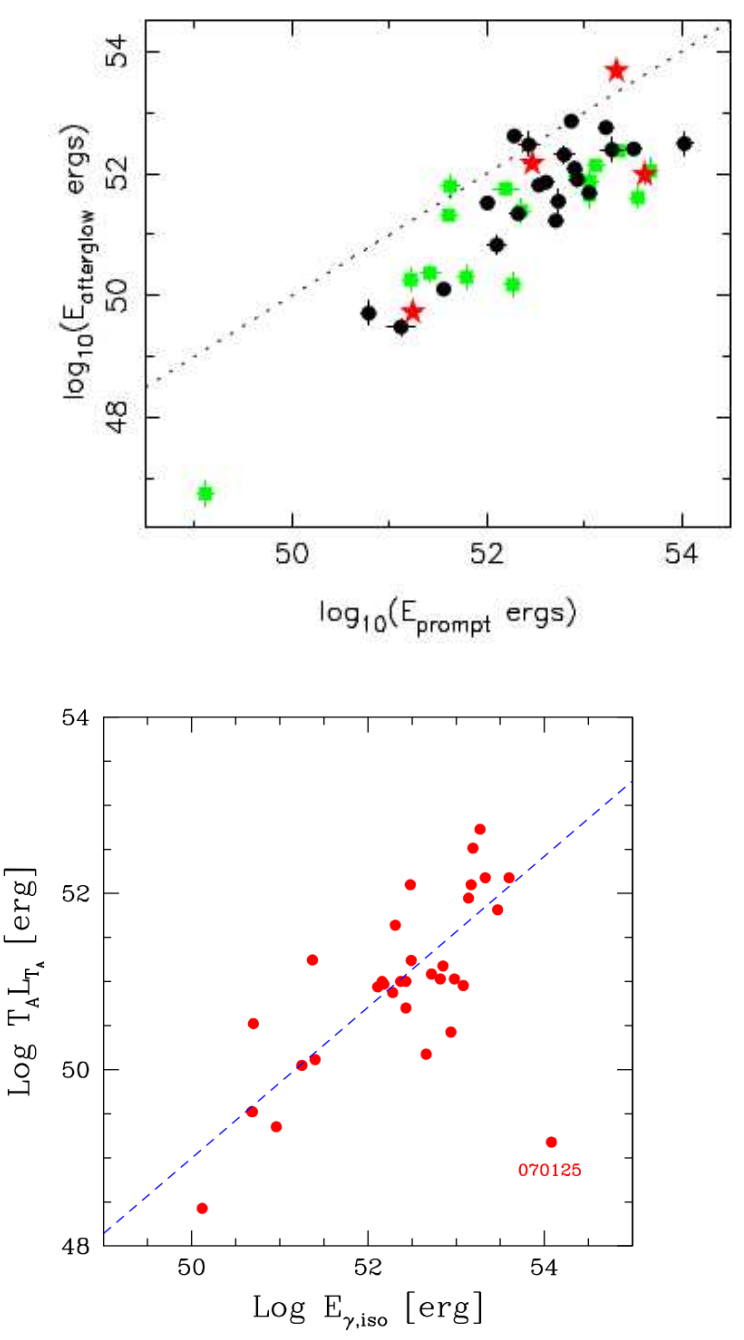

Figure 13. Upper left panel: the $F_{X, \text { afterglow }}$ in the XRT band (0.3-10 keV) vs. $F_{\gamma, \text { prompt }}$ computed from the BAT $T_{90}$ flux (15-150 $\mathrm{keV})$ plus the XRT flux $(0.3-10 \mathrm{keV})$ from Willingale et al. (2007). The dotted line represents where $F_{X, \text { afterglow }}$ and $F_{\gamma, p r o m p t}$ are identical. Upper right panel: $\log E_{\gamma, \text { prompt }}$ vs. $\log E_{X, \text { afterglow }}$ from Willingale et al. (2007). Symbols show the position of the afterglow in the $\beta_{X, a}-\alpha_{X, a}$ plane. GRBs that fall in the pre-jet-break region are plotted as dots, those that fall above this in the post-jet-break region are plotted as stars, and those below the pre-jet-break band are plotted as squares. The dotted line represents equality between $\log E_{\gamma, \text { prompt }}$ and $\log E_{X, \text { afterglow. }}$. Bottom left panel: the $\log E_{\gamma, \text { prompt }}-\log E_{X, \text { afterglow }}$ relation $\left(E_{\gamma, \text { iso }}\right.$ and $E_{X, i s o}$ respectively in the picture) from Liang et al. (2007). The solid line is the best fit. The dashed line indicates the $2 \sigma$ area. Bottom right panel: log $E_{X, p l a t e a u}$ vs. $\log E_{\gamma, \text { prompt }}$ from Ghisellini et al. (2009). The dashed line represents the least square fit with $\log E_{X, p l a t e a u}$ ( $T_{a} L_{T_{a}}$ in the picture) $\sim 0.86 \times \log E_{\gamma, \text { prompt }}\left(E_{\gamma, \text { iso }}\right.$ in the picture $)\left(P=2 \times 10^{-7}\right.$, without the outlier GRB 070125).

(LAT), on board the Fermi satellite ${ }^{4}$, they found that only $25 \%$ of the GBM bursts and none of the LAT bursts have $\eta<10 \%$. This implies that Fermi GRBs are more efficient at transferring kinetic energy into prompt radiation.

\footnotetext{
4 The Fermi Gamma ray Space Telescope (FGST), launched in 2008 and still running, is a space observatory being used to perform gamma ray astronomy observations from low Earth orbit. Its main instrument is the Large Area Telescope (LAT), an imaging gamma ray detector, (a pair-conversion instrument) which detects photons with energy from about $20 \mathrm{MeV}$ to $300 \mathrm{GeV}$ with a field of view of about $20 \%$ of the sky; it is a sequel to the EGRET instrument on the Compton gamma ray observatory (CGRO). Another instrument aboard Fermi is the Gamma Ray Burst Monitor (GBM), which is used to study prompt GRBs from $8 \mathrm{keV}$ to $30 \mathrm{MeV}$.
} 

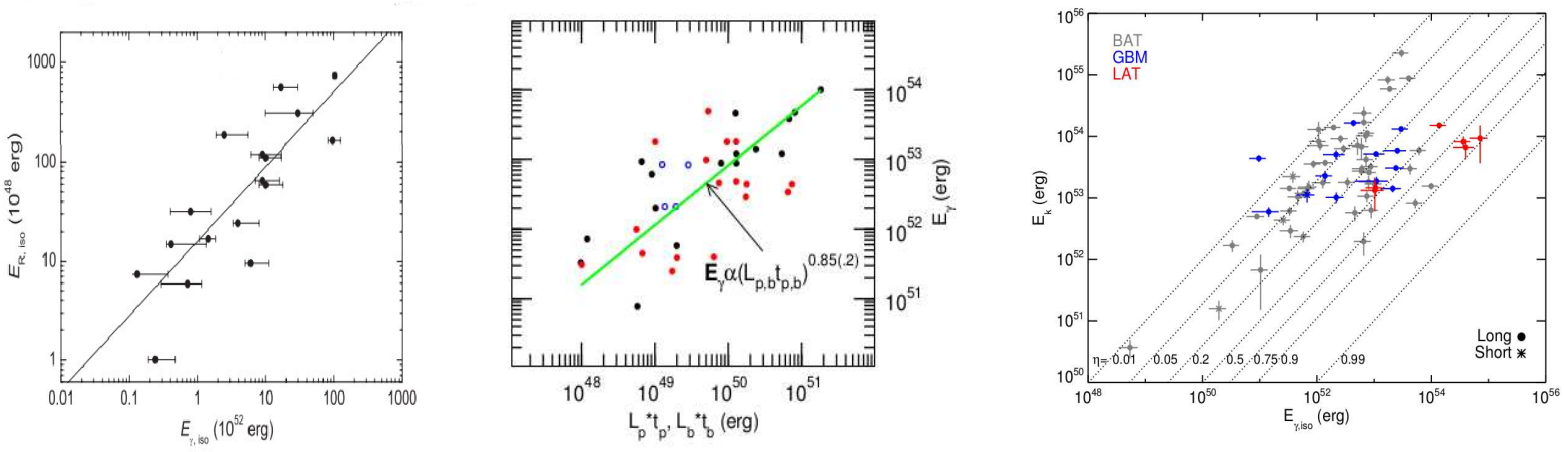

Figure 14. Left panel: "relation between $E_{\gamma, \text { prompt }}$ and $E_{O, \text { afterglow }}\left(E_{\gamma, \text { iso }}\right.$ and $E_{R, i s o}$ respectively in the picture), for the optically selected sample, from Liang et al. (2010). Line is the best fit". Middle panel: "relation between $\log E_{\gamma, \text { prompt }}$ and $\log E_{O, \text { afterglow }}$ ( $E_{\gamma, \text { iso }}$ and $L_{p} \times t_{p}$ respectively in the picture) from Panaitescu and Vestrand (2011). Black symbols are for afterglows with optical peaks, red symbols for optical plateaus, open circles for afterglows of uncertain type. $r\left(\log E_{X, \text { afterglow }}, \log E_{\gamma, p r o m p t}\right)=0.66$ for all 37 afterglows.

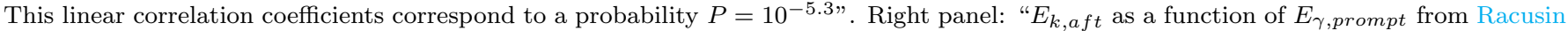
et al. (2011). The dashed lines indicated different values of $\eta$. The bursts detected by LAT on board of Fermi tend to have high $E_{\gamma, p r o m p t}$ ( $E_{\gamma, \text { iso }}$ in the picture), but average $E_{k, a f t}$, and therefore higher values of $\eta$ than the samples from BAT on board of Swift or GBM on board of Fermi".
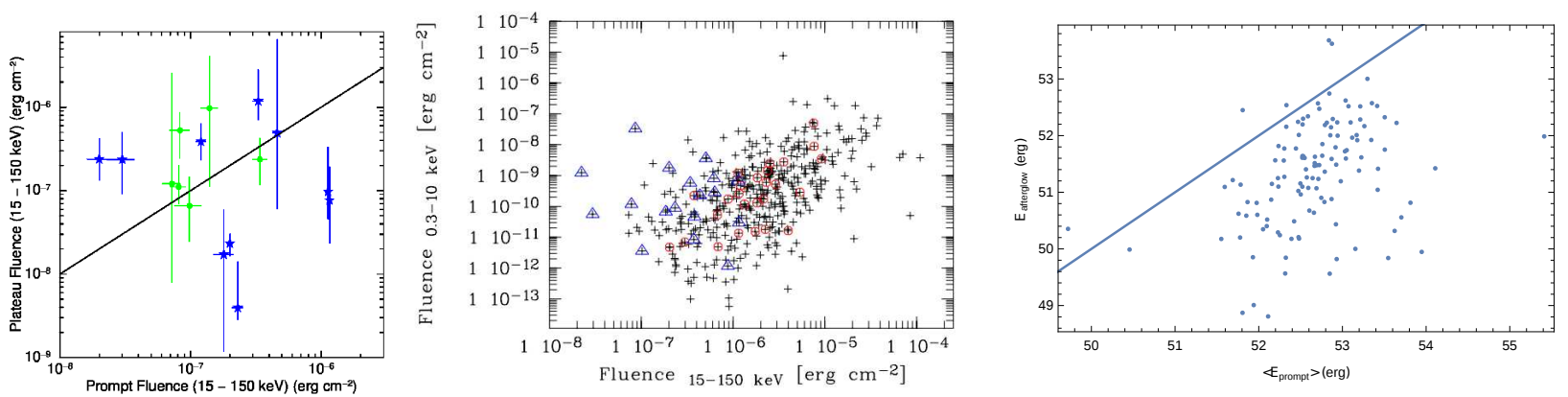

Figure 15. Left panel: the prompt BAT 15-150 keV fluence vs. the X-ray fluence in the 15-150 keV energy band from Rowlinson et al. (2013). Blue stars are GRBs with 2 or more breaks in their light curves, green circles have 1 break and red triangles have no significant breaks in their light curves. The black line indicates where the shallow decay phase fluence is equal to the prompt fluence. Middle panel: relations of the $0.3-10 \mathrm{keV}$ XRT fluence with fluence in the 15-150 keV BAT band from Grupe et al. (2013). Short bursts are represented with triangles and high-redshift $(\mathrm{z}>3.5)$ bursts with circles. Right panel: $\left\langle\log E_{\gamma, \text { prompt }}>\right.$ vs. $\log E_{X, \text { afterglow }}$ relation from Dainotti et al. (2015b) for 123 LGRBs. The solid line for equal $\log E_{\gamma, \text { prompt }}$ and $\log E_{X, \text { afterglow }}$ is given for reference.

\begin{tabular}{|c|c|c|c|c|c|c|}
\hline Correlations & Author & $\mathrm{N}$ & Slope & Norm & Corr.coeff. & $\mathrm{P}$ \\
\hline$E_{X, \text { afterglow }}-E_{\gamma, \text { prompt }}$ & Liang et al. (2007) & 53 & $1.00_{-0.16}^{+0.16}$ & $-0.50_{-8.10}^{+8.10}$ & 0.79 & $<10^{-4}$ \\
$E_{O, \text { afterglow }}-E_{\gamma, \text { prompt }}$ & Liang et al. (2010) & 32 & $0.76_{-0.14}^{+0.14}$ & $1.30_{-0.14}^{+0.14}$ & 0.82 & $<10^{-4}$ \\
& Panaitescu \& Vestrand (2011) & 37 & 1.18 & & 0.66 & $10^{-5.3}$ \\
\hline$E_{X, \text { plateau }}-E_{\gamma, \text { prompt }}$ & Ghisellini et al. (2009) & 33 & 0.86 & & & $2 \times 10^{-7}$ \\
\hline$E_{k, \text { aft }}-E_{\gamma, \text { prompt }}$ & Ghisellini et al. (2009) & 33 & 0.42 & & & $10^{-3}$ \\
\hline
\end{tabular}

Table 5. Summary of the relations in this section. The first column represents the relation in $\log$ scale, the second one the authors, and the third one the number of GRBs in the used sample. Afterwards, the fourth and fifth columns are the slope and normalization of the relation and the last two columns are the correlation coefficient and the chance probability, P.

\subsection{The $L_{X, \text { afterglow }}-E_{\gamma, \text { prompt }}$ relation and its physical interpretation}

Berger (2007) investigated the prompt and afterglow energies in the observed frame of 16 SGRBs. A large fraction of them $(80 \%)$ follows a linear relation between the prompt fluence in the gamma band, $S_{\gamma, \text { prompt }}$, in the BAT range and the X-ray flux at 1 day, $F_{X, 1 d}$, in the XRT band given by:

$$
\log F_{X, 1 \mathrm{~d}} \sim(1.01 \pm 0.09) \times \log S_{\gamma, \text { prompt }},
$$

with $\rho=0.86$ and $P=5.3 \times 10^{-5}$. Gehrels et al. (2008) confirmed his results investigating the same relation, but with X-ray fluxes at 11 hours, $F_{X, 11}$, see Fig. 16. 


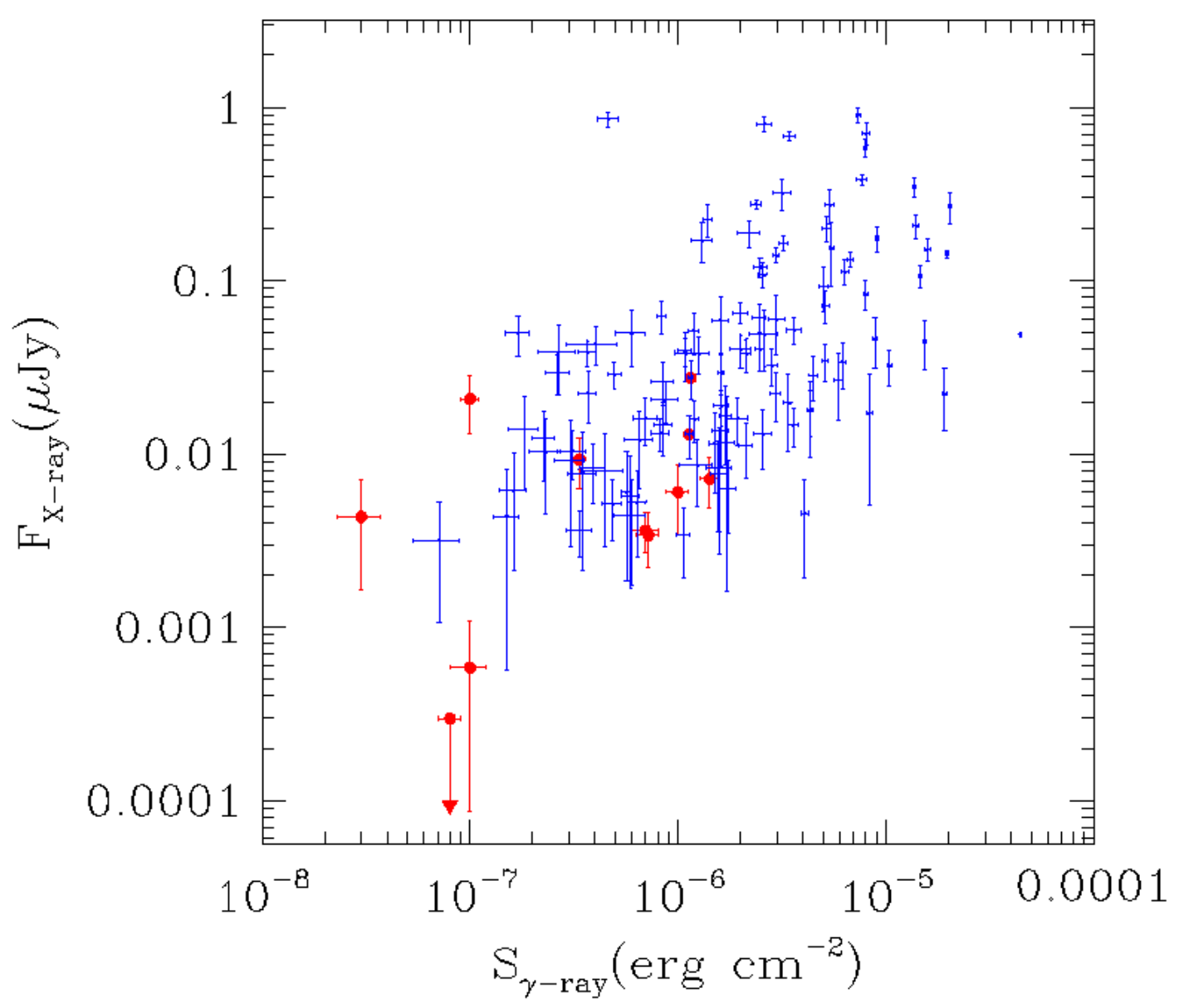

Figure 16. $F_{X, 11}-S_{\gamma, \text { prompt }}\left(F_{X-\text { ray }}\right.$ and $S_{\gamma-\text { ray }}$ respectively in the picture) relation for Swift SGRBs (in red) and LGRBs (in blue) from Gehrels et al. (2008). The XRT $F_{X, 11}$ are computed at $3 \mathrm{keV}$ and the BAT $S_{\gamma, \text { prompt }}$ are detected between 15 and $150 \mathrm{keV}$ (Sakamoto et al. 2008).

Later, Nysewander et al. (2009) considered the relation between $F_{X, 11}$ or the optical flux at 11 hours, $F_{O, 11}$, and $E_{\gamma, \text { prompt }}$, finding an almost linear relation, see Fig. 17. They used a data set of 37 SGRBs and 421 LGRBs detected by Swift. Panaitescu and Vestrand (2011) confirmed, in part, these results. They found a similar relation between $E_{\gamma, p r o m p t}$ and $F_{O, a}$ using $37 \mathrm{GRBs}$, but with a higher slope $(b=1.67)$, see the left panel of Fig. 18.

Kaneko et al. (2007) showed a linear relation $L_{X, 10} \propto E_{\gamma, \text { prompt }}$, where $L_{X, 10}$ is the X-ray luminosity at 10 hours calculated in the 2-10 keV energy range, while $E_{\gamma, \text { prompt }}$ in the 20-2000 keV energy range, see the left panel of Fig. 19. This relation compares four long events spectroscopically associated with SNe with "regular" energetic LGRBs $\left(E_{\gamma, \text { prompt }} \sim 10^{52}-10^{54} \mathrm{erg}\right.$ ). The results possibly indicate a common efficiency $\eta$ for transforming kinetic energy into gamma rays in the prompt phase for both these four events and for "regular" energetic LGRBs.

The same relation has been studied in the context of the low luminosity versus normal luminosity GRBs. Indeed, Amati et al. (2007) found that the relation between $L_{X, 10}$, in the 2-10 keV band, and $E_{\gamma, \text { prompt }}$, in the 1-10000 keV band, becomes stronger $\left(P \sim 10^{-11}\right)$ including sub-energetic GRBs as GRB 060218, GRB 980425 and GRB 031203, see the middle panel of Fig. 19. Therefore, it is claimed that sub-energetic GRBs are intrinsically faint and are considered to some extent normal cosmological GRBs.

Finally, Berger (2007) also analyzed the relation between the X-ray luminosity at one day, $L_{X, 1 \mathrm{~d}}$, and $E_{\gamma, p r o m p t}$, using 13 SGRBs with measured $z$. They found a slope $b=1.13 \pm 0.16$ (see the right panel of Fig. 18).

Liang et al. (2010) confirmed his results in the optical range using a sample of 32 Swift GRBs $\left(E_{\gamma, \text { prompt }}-L_{O, \text { peak }}\right.$ with $b=1.40 \pm 0.08$, see the right panel of Fig. 19). In addition, Kann et al. (2010) also confirmed his results with a 

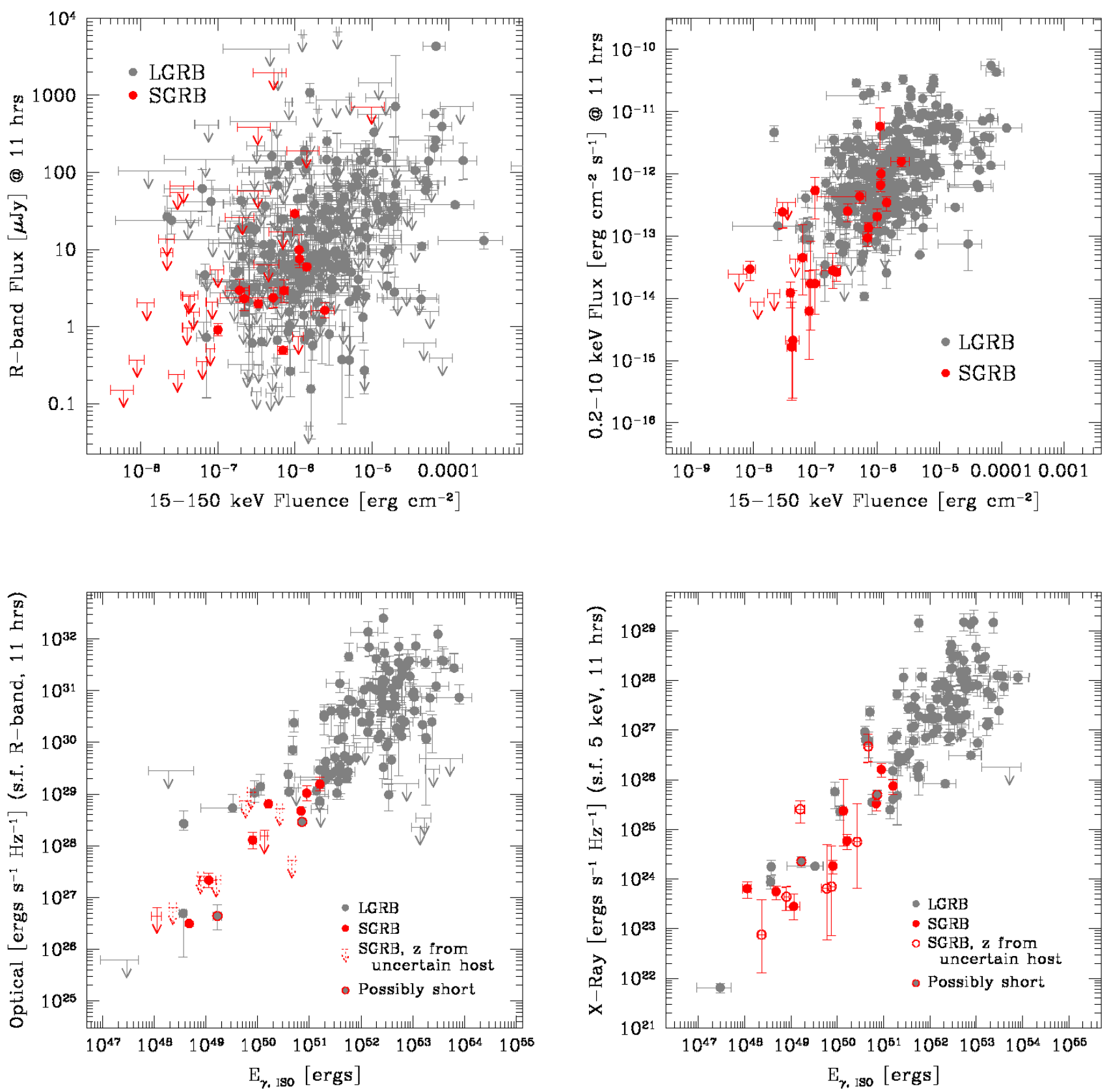

Figure 17. Upper left panel: "a plot of $F_{O, 11}$ (corrected for Galactic extinction) vs. 15-150 keV $S_{\gamma, p r o m p t}$ for both LGRBs (grey) and SGRBs (red) from Nysewander et al. (2009). Note that below a fluence of $10^{-7} \mathrm{erg} \mathrm{cm}^{-2}$, no optical afterglow of an SGRB has been discovered, while above $10^{-7}$, all reasonably deep observing campaigns, but one (GRB 061210) have detected an optical afterglow". Upper right panel: "a plot of $F_{X, 11}$ vs. $15-150 \mathrm{keV} S_{\gamma, \text { prompt }}\left(E_{\gamma, \text { iso }}\right.$ in the picture) for both LGRBs (grey) and SGRBs (red) from Nysewander et al. (2009)". Bottom left panel: "a plot of $L_{O, 11}$ (corrected for Galactic extinction) vs. E $E_{\gamma, p r o m p t}$ from Nysewander et al. (2009). Dashed upper limits represent SGRBs with a host galaxy determined by XRT error circle only. The classification of GRB 060614 and GRB 060505 is uncertain, therefore, they are labelled as "possibly short" ". Bottom right panel: "a plot of $L_{X, 11}$ vs. E $E_{\gamma, p r o m p t}$ from Nysewander et al. (2009). The open circles represent SGRBs with a host galaxy determined by XRT error circle only. The classification of GRB 060614 and GRB 060505 is uncertain, therefore, they are labelled as "possibly short" ".

sample of 76 LGRBs $\left(E_{\gamma, \text { prompt }}-L_{O, 1 \mathrm{~d}}\right.$ with $b=0.36$, see the left panel of Fig. 20).

Similarly, Dainotti et al. (2011b) analyzed the relation between $\log L_{X, a}$ and $\log E_{\gamma, p r o m p t}$ using the light curves of 66 LGRBs from the Swift BAT+XRT repository, http://www.swift.

ac.uk/burst_analyser/. Their sample has been divided into two subsamples: E4 formed of 62 LGRBs and E0095 consisting of 8 LGRBs, assuming $\sigma_{E}$ as a parameter representing the goodness of the fit. For the E4 subsample it was 

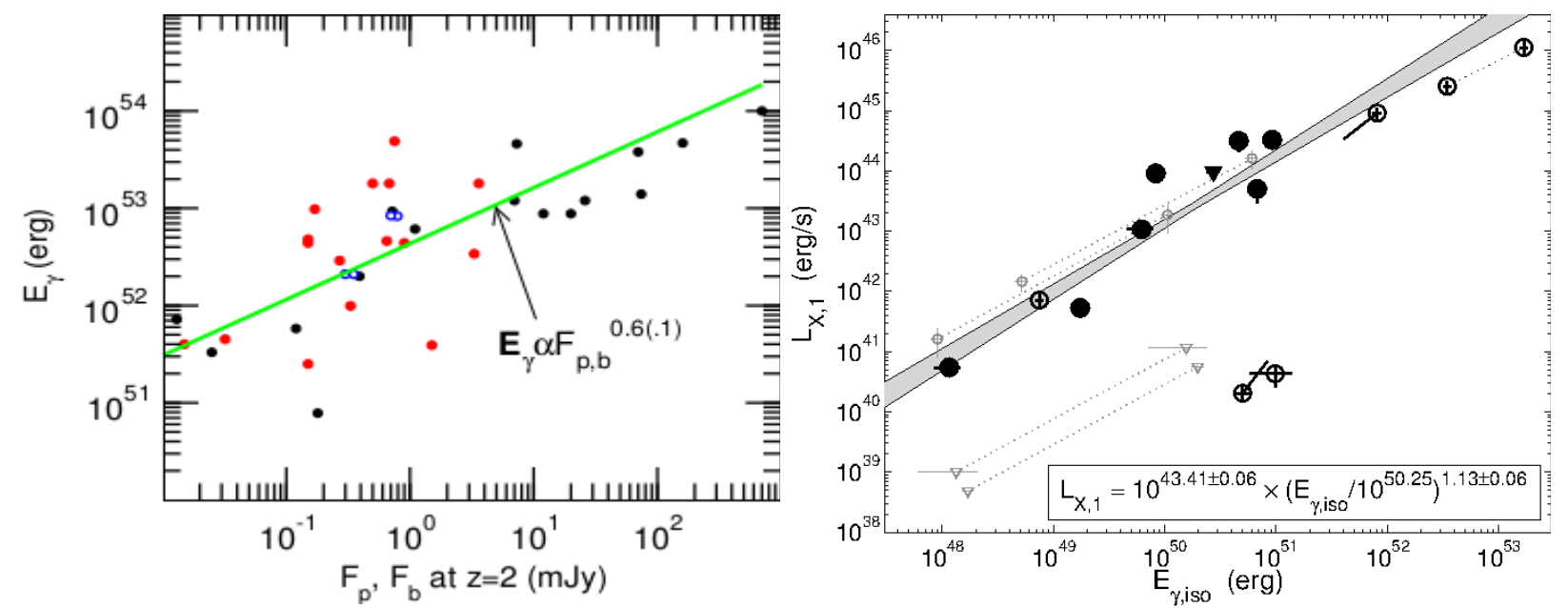

Figure 18. Left panel: $E_{\gamma, \text { prompt }}-F_{O, a}\left(E_{\gamma, \text { iso }}\right.$ and $F_{p}$ respectively in the picture) relation from Panaitescu and Vestrand (2011). Black symbols are for afterglows with optical peaks, red symbols for optical plateaus, open circles for afterglows of unknown kind. Right panel: $L_{X, 1 \mathrm{~d}}$ vs. $E_{\gamma, \text { prompt }}\left(E_{\gamma, i s o}\right.$ in the picture) for the SGRBs with a known $z$ (solid black circles), redshift constraints (open black circles) and without any redshift information (grey symbols connected by dotted lines) from Berger (2007).
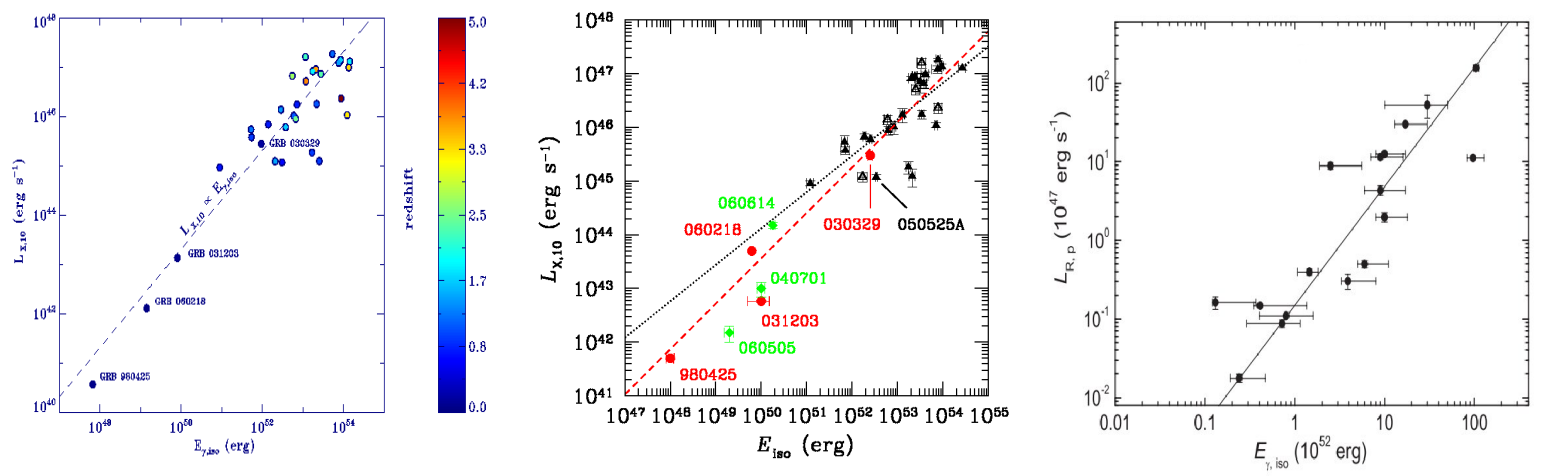

Figure 19. Left panel: " $L_{\mathrm{X}, 10}$ of SN-GRBs (source frame: $2-10 \mathrm{keV}$ ) as a function of their $E_{\gamma, \text { prompt }}$, $E_{\gamma, \text { iso }}$ in the picture, (20-2000 $\mathrm{keV}$ ) from Kaneko et al. (2007). $z$ for each event is also shown in colour". Middle panel: " $L_{X, 10}$ (in 2-10 keV range) vs. $E_{\gamma, p r o m p t}\left(E_{i s o}\right.$ in the picture) for the events included in the sample of Nousek et al. (2006) (triangles) plus the 3 sub-energetic GRB 980425, GRB 031203, GRB 060218, the other GRB/SN event GRB 030329 (circles), and 3 GRBs with known $z$ and deep limits to the peak magnitude of associated SN, XRF 040701, GRB 060505 and GRB 060614 (diamonds) from Amati et al. (2007). Empty triangles indicate those GRBs for which the 1-10000 keV $E_{\gamma, \text { prompt }}$ was computed based on the 100-500 keV $E_{\gamma, \text { prompt }}$ reported by Nousek et al. (2006) by assuming an average spectral index. The plotted lines are the best-fit power laws obtained without (dotted) and with (dashed) sub-energetic GRBs and GRB 030329". Right panel: "relation between $E_{\gamma, p r o m p t}$ and $L_{O, p e a k}\left(E_{\gamma, i s o}\right.$ and $L_{R, p}$ respectively in the picture) for the optically selected sample from Liang et al. (2010). Line is the best fit".

found:

$$
\log L_{X, a}=28.03_{-2.97}^{+2.98}+0.52_{-0.06}^{+0.07} \times \log E_{\gamma, p r o m p t},
$$

with $\rho=0.43$ and $P=1.4 \times 10^{-5}$, while for the E0095 subsample

$$
\log L_{X, a}=29.82_{-7.82}^{+7.11}+0.49_{-0.16}^{+0.21} \times \log E_{\gamma, \text { prompt }},
$$

with $\rho=0.83$ and $P=3.2 \times 10^{-2}$. Thus, it was concluded that the small error energy sample led to a higher relation and to the existence of a subset of GRBs which can yield a "standardizable candle". Furthermore, $\operatorname{since} \log L_{X, a}$ and $\log T_{X, a}^{*}$ are strongly correlated, and the slope is roughly -1 , the energy reservoir of the plateau is roughly constant. Since $\log E_{\gamma, \text { peak }}$ and $\log E_{\gamma, \text { prompt }}$ are both linked with $\log L_{X, a}$, then the $\log E_{\gamma, \text { peak }}-\log E_{\gamma, \text { prompt }}-\log E_{X, p l a t e a u}$ relation is straightforward. For its modification taking into account $\log E_{\gamma, \text { iso }}$ of the whole X-ray light curves see Bernardini et al. (2012b). As further confirmations of the $L_{X, a}-E_{\gamma, \text { prompt }}$ relation, D'Avanzo et al. (2012) and Margutti et al. (2013) found a relation between $\log L_{X, a}$ and $E_{\gamma, \text { prompt }}$ with slope $b \sim 1$ and $\rho \approx 0.70$, using 58 and 297 Swift LGRBs respectively.

Furthermore, Berger (2014) studied the relation between the X-ray luminosities at 11 hours, $L_{X, 11}$, and $E_{\gamma, p e a k}$, and 

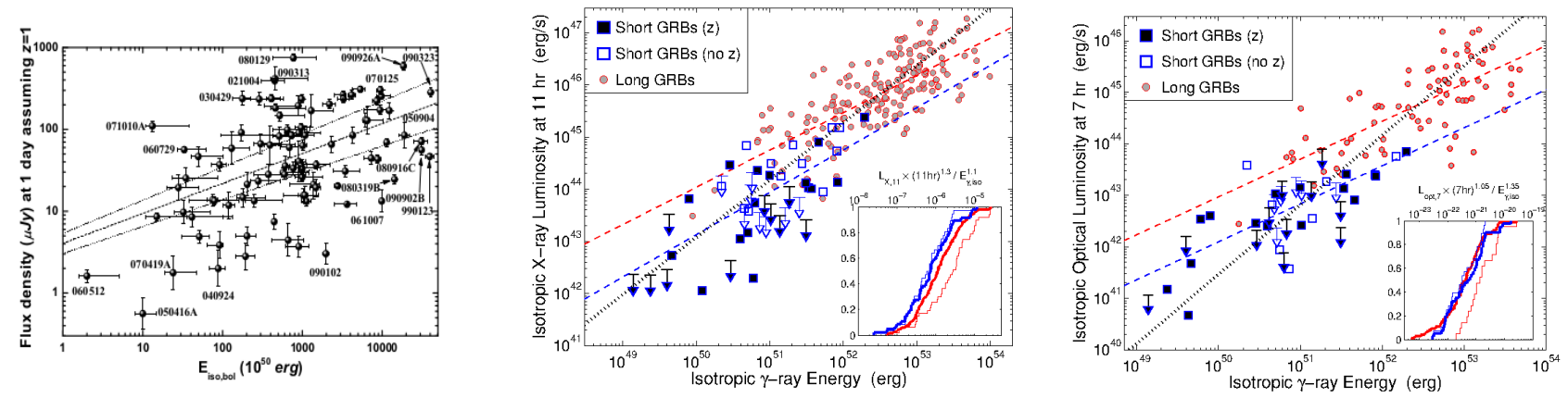

Figure 20. Left panel: " $F_{O, 1 \mathrm{~d}}$ in the $R$ band plotted against the bolometric $E_{\gamma, p r o m p t}\left(E_{i s o, b o l}\right.$ in the picture) for all GRBs in the optically selected sample from Kann et al. (2010) (except GRB 991208, which was only discovered after several days, and GRBs 060210, $060607 \mathrm{~A}, 060906$ and $080319 \mathrm{C}$, where the follow-up does not extend to one day). While no tight relation is visible, there is a trend of increasing optical luminosity with increasing prompt energy release. This is confirmed by a linear fit (in log-log space), using a Monte Carlo analysis to account for the asymmetric errors. The dashed line shows the best fit, while the dotted line marks the $3 \sigma$ error region. Several special GRBs are marked". Middle panel: " $L_{X, 11}$ vs. $E_{\gamma, p r o m p t}$ for SGRBs (blue) and LGRBs (grey) from Berger (2014). Open symbols for SGRBs indicate events without a known $z$, for which a fiducial value of $z=0.75$ is assumed. The dashed blue and red lines are the best-fit power law relations to the trends for SGRBs and LGRBs, respectively, while the dotted black line is the expected relation based on the afterglow synchrotron model with $\nu_{X}>\nu_{c}$ and $p=2.4\left(\log L_{X, 11} \sim 1.1 \times \log E_{\gamma, p r o m p t}\right)$. The inset shows the distribution of the ratio $\log \left(L_{X, 11} \times(11 \mathrm{hr})^{1.3} / E_{\gamma, \text { prompt }}^{1.1}\right)$, for the full samples (thick lines) and for the region where SGRBs and LGRBs have equal $E_{\gamma, \text { prompt }}$ values (thin lines). The lower level of $L_{X, 11}$ relative to $E_{\gamma, p r o m p t}$ for SGRBs is evident from these various comparisons". Right panel: same as in the middle panel, "but for the isotropic-equivalent afterglow optical luminosity at a rest frame time of 7 hours $\left(L_{O, 7}\right)$, still from Berger (2014). The dotted black line is the expected relation based on the afterglow model for $\nu_{m}<\nu_{O}<\nu_{c}$ and $p=2.4$ $\left(\log L_{O, 7} \sim 1.35 \times \log E_{\gamma, \text { prompt }}\right)$. The inset shows the distribution of the ratio $\log \left(L_{O, 7} \times(7 \mathrm{hr})^{1.05} / E_{\gamma, \text { prompt }}^{1.35}\right)$, for the full samples (thick lines) and for the region where SGRBs and LGRBs have equal $E_{\gamma, \text { prompt }}$ values (thin lines). The lower level of $L_{O, 7}$ relative to $E_{\gamma, p r o m p t}$ for SGRBs is evident from these various comparisons".
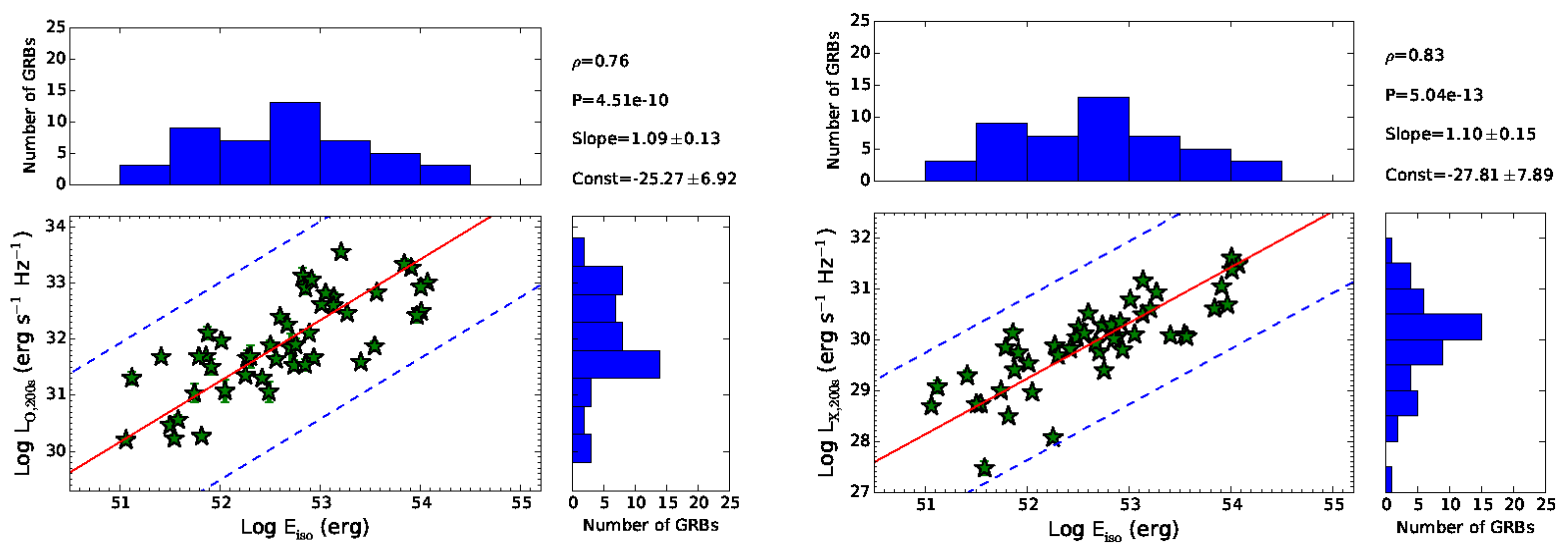

Figure 21. Left panel: $\log L_{O, 200 s^{-}} \log E_{\gamma, \text { prompt }}\left(E_{\text {iso }}\right.$ in the picture) relation from Oates et al. (2015). Right panel: $\log L_{X, 200 s^{-}}$ $\log E_{\gamma, \text { prompt }}\left(E_{\text {iso }}\right.$ in the picture) relation from Oates et al. (2015).

the relation between the optical luminosity at 7 hours $L_{O, 7}$ and $E_{\gamma, p e a k}$ for a sample of 70 SGRBs and 73 LGRBs detected mostly by Swift. He found that the observed relations are flatter than the ones simulated by Kann et al. (2010), see the middle and right panels of Fig. 20.

Regarding the relation between $E_{\gamma, \text { prompt }}$ and the optical luminosities, Oates et al. (2015) analyzed the relation between $L_{O, 200 \mathrm{~s}}$ or $L_{X, 200 \mathrm{~s}}$ and $\log E_{\gamma, p r o m p t}$ with a sample of 48 LGRBs. They claimed a strong connection between prompt and afterglow phases, see Fig. 21 and Table 6. This relation permits to study some important spectral characteristics of GRBs, the optical and X-ray components of the radiation process and the standard afterglow model. In Table 6, a summary of the relations described in this section is shown.

Regarding the physical interpretation of the $L_{X, \text { afterglow }}-E_{\gamma, \text { prompt }}$ relation, Gehrels et al. (2008) underlined that the optical and X-ray radiation are characterized by $\beta_{O X, a} \approx 0.75$. This value matches the slow cooling case, important at 11 hours, when the electron distribution power law index is $p=2.5$ for $\nu_{m}<\nu_{O}<\nu_{X}<\nu_{c}$.

Oates et al. (2015) pointed out that within the standard afterglow model, the $\log E_{\gamma, \text { prompt }}-\left(\log L_{O, 200 \mathrm{~s}}, \log L_{X, 200 \mathrm{~s}}\right)$ relations are expected. However, the slopes of the simulated and observed relations are inconsistent at $>3 \sigma$ due to 
values set for the $\eta$ parameter. If the distribution of the efficiencies is not sufficiently narrow the relation will be more disperse. Thus, the simulations repeated with $\eta=0.1$ and $\eta=0.9$ gave, anyway, incompatible results between the simulated and observed slopes at $>3 \sigma$.

\begin{tabular}{|c|c|c|c|c|c|c|}
\hline Correlations & Author & $\mathrm{N}$ & Slope & Norm & Corr.coeff. & $\mathrm{P}$ \\
\hline$F_{X, 1 \mathrm{~d}}-S_{\gamma, \text { prompt }}$ & Berger (2007) & 16 & $1.01_{-0.09}^{+0.09}$ & & 0.86 & $5.3 \times 10^{-5}$ \\
\hline \multirow{2}{*}{$F_{X, 11}-S_{\gamma, \text { prompt }}$} & Gehrels et al. (2008) & 111 & $0.63_{-0.04}^{+0.04}$ & $2.11_{-0.21}^{+0.21}$ & 0.53 & $4 \times 10^{-9}$ \\
\hline & Gehrels et al. (2008) & 10 & $0.36_{-0.17}^{+0.17}$ & $0.06_{-1.07}^{+1.07}$ & 0.35 & 0.31 \\
\hline \multirow{2}{*}{$\begin{array}{l}F_{O, 11}-E_{\gamma, p r o m p t} \\
F_{O, 11}-E_{\gamma, p r o m p t}\end{array}$} & Nysewander et al. (2009) & 421 & $\sim 1$ & & & \\
\hline & Nysewander et al. (2009) & 37 & $\sim 1$ & & & \\
\hline \multirow{2}{*}{$\begin{array}{l}F_{X, 11}-E_{\gamma, \text { prompt }} \\
F_{X, 11}-E_{\gamma, \text { prompt }}\end{array}$} & Nysewander et al. (2009) & 421 & $\sim 1$ & & & \\
\hline & Nysewander et al. (2009) & 37 & $\sim 1$ & & & \\
\hline$F_{O, a}-E_{\gamma, p r o m p t}$ & Panaitescu\&Vestrand (2011) & 37 & 1.67 & & 0.75 & $10^{-7.3}$ \\
\hline$L_{X, 1 \mathrm{~d}}-E_{\gamma, \text { prompt }}$ & Berger $(2007)$ & 13 & $1.13_{-0.16}^{+0.16}$ & $43.43_{-0.20}^{+0.20}$ & 0.94 & $3.2 \times 10^{-6}$ \\
\hline$L_{O, \text { peak }}-E_{\gamma, \text { prompt }}$ & Liang et al. (2010) & 32 & $1.40_{-0.08}^{+0.08}$ & $0.83_{-0.15}^{+0.15}$ & 0.87 & $10^{-4}$ \\
\hline$L_{O, 1 \mathrm{~d}}-E_{\gamma, \text { prompt }}$ & Kann et al. (2010) & 76 & 0.36 & & & \\
\hline \multirow[t]{4}{*}{$L_{X, a}-E_{\gamma, \text { prompt }}$} & Dainotti et al. (2011b) & 62 & $0.52_{-0.06}^{+0.07}$ & $28.03_{-2.97}^{+2.98}$ & 0.43 & $1.4 \times 10^{-5}$ \\
\hline & Dainotti et al. (2011b) & 8 & $0.49_{-0.16}^{+0.21}$ & $29.82_{-7.82}^{+7.11}$ & 0.83 & $3.2 \times 10^{-2}$ \\
\hline & D'Avanzo et al. (2012) & 58 & $\sim 1$ & & $\approx 0.70$ & \\
\hline & Margutti et al. (2013) & 297 & $\sim 1$ & & $\approx 0.70$ & \\
\hline \multirow[t]{2}{*}{$L_{X, 11}-E_{\gamma, \text { prompt }}$} & Berger $(2014)$ & 73 & 0.72 & 44.75 & & \\
\hline & Berger (2014) & 70 & 0.83 & 43.93 & & \\
\hline \multirow[t]{2}{*}{$L_{O, 7}-E_{\gamma, p r o m p t}$} & Berger (2014) & 73 & 0.73 & 43.70 & & \\
\hline & Berger (2014) & 70 & 0.74 & 42.84 & & \\
\hline$L_{X, 200 \mathrm{~s}}-E_{\gamma, \text { prompt }}$ & Oates et al. (2015) & 48 & $1.10_{-0.15}^{+0.15}$ & $\begin{array}{l}-27.81_{-7.89}^{+7.89} \\
\end{array}$ & 0.83 & $5.04 \times 10^{-13}$ \\
\hline$L_{O, 200 \mathrm{~s}}-E_{\gamma, \text { prompt }}$ & Oates et al. (2015) & 48 & $1.09_{-0.13}^{+0.13}$ & $-25.27_{-6.92}^{+6.92}$ & 0.76 & $4.51 \times 10^{-10}$ \\
\hline
\end{tabular}

Table 6. Summary of the relations in this section. The first column represents the relation in $\log$ scale, the second one the authors, and the third one the number of GRBs in the used sample. Afterwards, the fourth and fifth columns are the slope and normalization of the relation and the last two columns are the correlation coefficient and the chance probability, P.

\subsection{The $L_{X, a}-L_{O, a}$ relation and its physical interpretation}

In the observed frame, Jakobsson et al. (2004) studied the $\log F_{O, 11}$ versus $\log F_{X, 11}$ distribution, in the optical $\mathrm{R}$ band and in the $2-10 \mathrm{keV}$ band respectively, using all known GRBs with a detected X-ray afterglow, see the left panel of Fig. 22. Different from the previous definition of dark bursts (where dark bursts were simply defined as those bursts in which the optical transient is not observed), they defined these bursts as GRBs where the optical-to-X-ray spectral index, $\beta_{O X, a}$, is shallower than the X-ray spectral index minus $0.5, \beta_{X, a}-0.5$. They found out 5 dark bursts among 52 observed by Beppo-SAX ${ }^{5}$. This analysis aimed at distinguishing dark GRBs through Swift. Gehrels (2007) and Gehrels et al. (2008) confirmed the results using a data sample of 19 SGRBs and 37 LGRBs+6 SGRBs respectively, see the middle and right panels of Fig. 22. In particular, Gehrels et al. (2008) obtained a slope $b=0.38 \pm 0.03$ for LGRBs and $b=0.14 \pm 0.45$ for SGRBs.

Instead in the rest-rest frame, Berger (2014) studied the relation between $L_{O, 7}$ and $L_{X, 11}$ on 70 SGRBs and 73 LGRBs, finding some similarities between SGRBs and LGRBs and a central value $\left\langle L_{O, 7} / L_{X, 11}\right\rangle \approx 0.08$, see the left panel of Fig. 23.

Oates et al. (2015) improved their study. They analyzed a similar relation with a sample of 48 LGRBs, but using $L_{O, 200 \mathrm{~s}}$ and $L_{X, 200 \mathrm{~s}}$, see the right panel of Fig. 23. The slope obtained has a value $b=0.91 \pm 0.22$.

This relation helps to explore the synchrotron spectrum of GRBs and to obtain some constraints on the circumburst medium for both LGRBs and SGRBs. In Table 7 a summary of the relations described in this section is displayed.

\footnotetext{
${ }^{5}$ Beppo-SAX, (1996-2003), was an Italian-Dutch satellite capable of simultaneously observing targets over more than 3 decades of energy, from 0.1 to $600 \mathrm{keV}$ with relatively large area, good (for that time) energy resolution and imaging capabilities (with a spatial resolution of 1 arcminute between 0.1 and $10 \mathrm{keV}$ ). The instruments on board Beppo-SAX are Low Energy Concentrator Spectrometer (LECS), Medium Energy Concentrator Spectrometer (MECS), High Pressure Gas Scintillation Proportional Counter (HPGSPC), Phoswich Detector System (PDS) and Wide Field Camera (WFC, from 2-30 keV and from $100-600 \mathrm{keV}$ ). The first four instruments point to the same direction allowing observations in the broad energy range $(0.1-300 \mathrm{keV})$. With the WFC it was possible to model the afterglow as a simple power law, mainly due to the lack of observations during a certain period in the GRB light curve.
} 

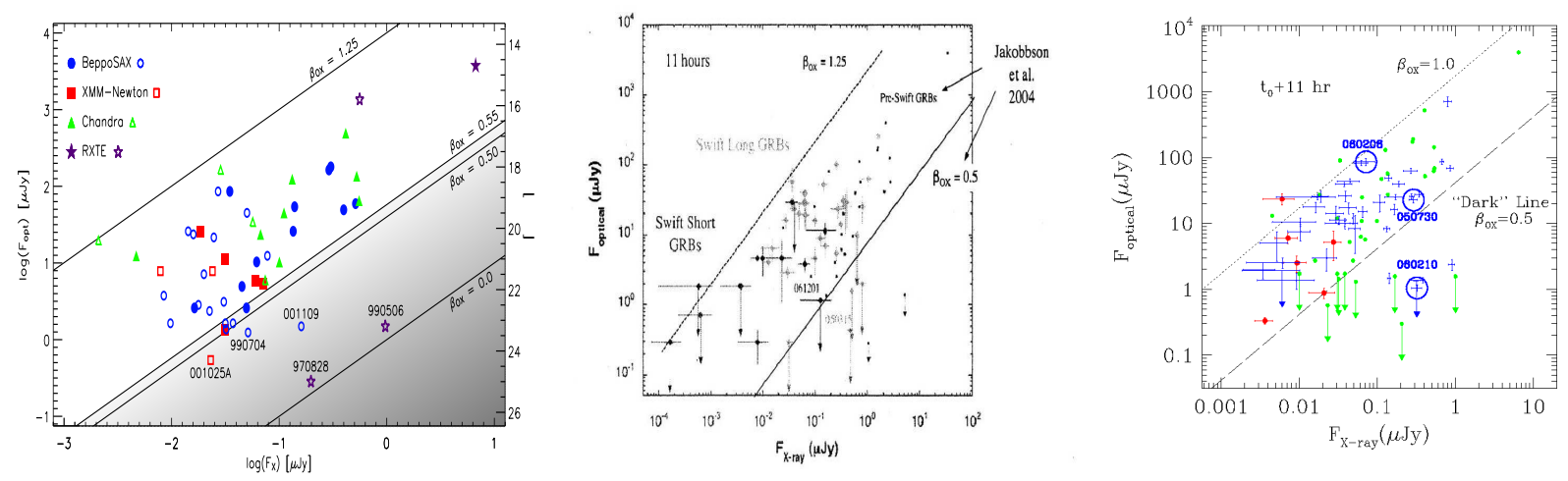

Figure 22. Left panel: $\log F_{O, 11}-\log F_{X, 11}\left(F_{o p t}\right.$ and $F_{X}$ respectively in the plot) distribution for the data set from Jakobsson et al. (2004). Filled symbols show optical detections while open symbols represent upper limits. Lines of constant $\beta_{O X, a}$ are displayed with the corresponding value. Dark bursts are those which have $\beta_{O X, a}<0.5$. Middle panel: $F_{X, 11}-F_{O, 11}$ relation for Swift SGRBs and LGRBs from Gehrels (2007). Comparison is made to pre-Swift GRBs and to lines of optical to X-ray spectral index from Jakobsson et al. (2004). The grey points indicate LGRBs, the black points represent SGRBs and the small black points without error bars are the pre-Swift GRBs. Right panel: $F_{O, 11}-F_{X, 11}$ relation for Swift SGRBs (shown in red) and LGRBs (shown in blue) from Gehrels et al. (2008). The three circled bursts are those for which $z>3.9$. The pre-Swift GRBs taken from Jakobsson et al. (2004) are presented in green. Also the dark burst separation line $\beta_{O X, a}=0.5$ (Jakobsson et al. 2004) and a line showing $\beta_{O X, a}=1.0$ are represented.
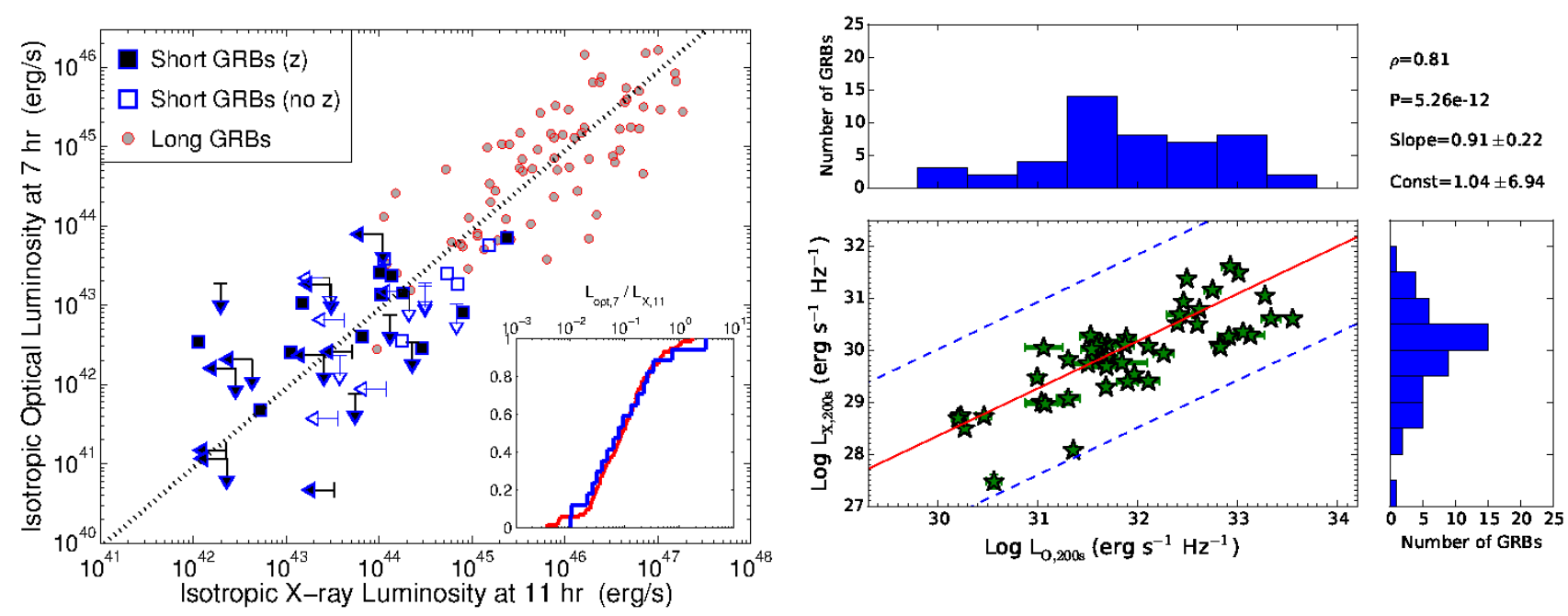

Figure 23. Left panel: " $L_{O, 7}$ vs. $L_{X, 11}$ from Berger (2014). The dotted black line marks a linear relation, expected for $\nu_{X} \sim \nu_{c}$. The inset shows the distribution of the ratio $L_{O, 7} / L_{X, 11}$, indicating that both SGRBs and LGRBs exhibit a similar ratio, and that in general

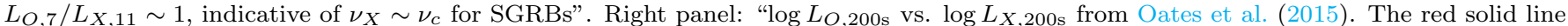
represents the best fit regression and the blue dashed line represents 3 times the RMS deviation. In the top right corner, it is given $\rho$ and $P$ and it is provided the best-fit slope and constant determined by linear regression".

\begin{tabular}{|c|c|c|c|c|c|c|}
\hline Correlations & Author & $\mathrm{N}$ & Slope & Norm & Corr.coeff. & $\mathrm{P}$ \\
\hline$F_{X, 11}-F_{O, 11}$ & Gehrels et al. (2008) & 6 & $0.14 \pm 0.45$ & $0.72 \pm 0.94$ & 0.06 & 0.68 \\
& & 37 & $0.38 \pm 0.03$ & $1.62 \pm 0.04$ & 0.44 & 0.006 \\
\hline$L_{X, 11}-L_{O, 7}$ & Berger (2014) & 70 & 0.08 & & & \\
& & 73 & 0.08 & & & \\
\hline$L_{X, 200 \mathrm{~s}}-L_{O, 200 \mathrm{~s}}$ & Oates et al. (2015) & 48 & $0.91 \pm 0.22$ & $1.04 \pm 6.94$ & 0.81 & $5.26 \times 10^{-12}$ \\
\hline
\end{tabular}

Table 7. Summary of the relations in this section. The first column represents the relation in log scale, the second one the authors, and the third one the number of GRBs in the used sample. Afterwards, the fourth and fifth columns are the slope and normalization of the relation and the last two columns are the correlation coefficient and the chance probability, P. 
Regarding the physical interpretation of the $L_{X, a}-L_{O, a}$ relation, Berger (2014) showed that, in the context of the synchrotron model, the comparison of $L_{O, 7}$ and $L_{X, 11}$ indicated that usually $\nu_{c}$ is near or higher than the X-ray band. Indeed, LGRBs have often greater circumburst medium densities (about 50 times greater than SGRBs) and therefore $\nu_{c} \sim \nu_{X}$.

\subsection{The $L_{X}\left(T_{a}\right)-L_{\gamma, \text { iso }}$ relation}

In Dainotti et al. (2011b) the connections between the physical properties of the prompt emission and $\log L_{X, a}$ were analyzed using a sample of 62 Swift LGRBs. A relation was found between $\log L_{X, a}$ in the XRT band and the isotropic prompt luminosity, $\log <L_{\gamma, \text { iso }}>_{45} \equiv \log \left(E_{\gamma, p r o m p t} / T_{45}\right)$, in the BAT energy band, see the left panel of Fig. 24. This relationship can be fitted with the following equation:

$$
\log L_{X, a}=20.58_{-6.73}^{+6.66}+0.67_{-0.15}^{+0.14} \times \log <L_{\gamma, i s o}>_{45},
$$

obtaining $\rho=0.59$ and $P=7.7 \times 10^{-8}$. In this paper $\log L_{X, a}$ was related to several prompt luminosities defined using different time scales, such as $T_{90}, T_{45}$ (the time in which the $45 \%$ between $5 \%-50 \%$ of radiation is emitted in the prompt emission), and $T_{X, p}$ (the time at the end of the prompt emission within the W07 model). Furthermore, the E4 (defined in Table 1) subsample of 62 LGRBs with known $z$ from a sample of 77 Swift LGRBs and the E0095 subsample of 8 GRBs with smooth light curves were used, see black and red points in the left panel of Fig. 24.

Therefore, it has been shown that the GRB subsample with the strongest correlation coefficient for the LT relation also implies the tightest prompt-afterglow relations. This subsample can be used as a standard one for astrophysical and cosmological studies.

In the middle panel of Fig. 24, the correlation coefficients $\rho$ are shown for the following distributions: $\left(\log <L_{\gamma, i s o}>_{45}\right.$ , $\left.\log <L_{\gamma, \text { iso }}>_{90}, \log <L_{\gamma, i s o}>_{T_{X, p}}, \log E_{\gamma, p r o m p t}\right)-\log L_{X, a}$, represented by different colours, namely red, black, green and blue respectively.

No significant relations for the IC bursts have been found out. However, the paucity of the data does not allow a definitive statement. From this analysis, it is clear that the inclusion of the IC GRB class does not strengthen the existing relations.

\begin{tabular}{|c|c|c|c|c|}
\hline \multirow{3}{*}{ Correlations } & \multicolumn{2}{|r|}{$\mathrm{E} 4$} & \multicolumn{2}{|r|}{ E0095 } \\
\hline & & $(b, a)$ & 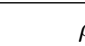 & $(b, a)$ \\
\hline & & $\mathrm{P}$ & & $\mathrm{P}$ \\
\hline \multirow[t]{2}{*}{$L_{X, a}-<L_{\gamma, \text { iso }}>_{45}$} & 0.59 & $\left(0.67_{-0.15}^{+0.14}, 20.58_{-6.73}^{+6.66}\right)$ & 0.95 & $\left(0.73_{-0.11}^{+0.16}, 17.90_{-6.0}^{+5.29}\right)$ \\
\hline & 0.62 & $7.7 \times 10^{-8}$ & 0.90 & $2.3 \times 10^{-3}$ \\
\hline \multirow[t]{2}{*}{$L_{X, a}-<L_{\gamma, i s o}>_{90}$} & 0.60 & $\left(0.63_{-0.16}^{+0.15}, 22.05_{-7.31}^{+7.14}\right)$ & 0.93 & $\left(0.84_{-0.12}^{+0.11}, 11.86_{-3.44}^{+3.43}\right)$ \\
\hline & 0.62 & $7.7 \times 10^{-8}$ & 0.94 & $2.7 \times 10^{-3}$ \\
\hline \multirow[t]{2}{*}{$L_{X, a}-<L_{\gamma, i \text { so }}>_{T_{X, p}}$} & 0.46 & $\left(0.73_{-0.14}^{+0.09}, 16.61_{-4.35}^{+4.35}\right)$ & 0.95 & $\left(0.93_{-0.23}^{+0.20}, 7.70_{-3.46}^{+3.47}\right)$ \\
\hline & 0.56 & $2.21 \times 10^{-6}$ & 0.90 & $2.3 \times 10^{-3}$ \\
\hline \multirow[t]{2}{*}{$L_{X, a}-E_{\gamma, p r o m p t}$} & 0.43 & $\left(0.52_{-0.06}^{+0.07}, 28.03_{-2.97}^{+2.98}\right)$ & 0.83 & $\left(0.49_{-0.16}^{+0.21}, 29.82_{-7.82}^{+7.11}\right)$ \\
\hline & 0.52 & $1.4 \times 10^{-5}$ & 0.75 & $3.2 \times 10^{-2}$ \\
\hline \multirow[t]{2}{*}{$T_{X, a}^{*}-E_{\gamma, \text { prompt }}$} & -0.19 & $\left(-0.49_{-0.08}^{+0.09}, 54.51_{-0.30}^{+0.37}\right)$ & -0.81 & $\left(-0.96_{-0.22}^{+0.21}, 54.67_{-0.69}^{+0.69}\right)$ \\
\hline & -0.21 & $1.0 \times 10^{-1}$ & -0.69 & $5.8 \times 10^{-2}$ \\
\hline \multirow[t]{2}{*}{$L_{X, a}-E_{\gamma, \text { peak }}$} & 0.54 & $\left(1.06_{-0.23}^{+0.53}, 43.88_{-1.00}^{+0.61}\right)$ & 0.74 & $\left(1.5_{-0.94}^{+0.65}, 43.10_{-2.26}^{+2.53}\right)$ \\
\hline & 0.51 & $2.2 \times 10^{-5}$ & 0.80 & $1.7 \times 10^{-2}$ \\
\hline \multirow[t]{2}{*}{$T_{X, a}^{*}-E_{\gamma, \text { peak }}$} & -0.36 & $\left(-0.66_{-0.29}^{+0.20}, 4.96_{-0.80}^{+0.81}\right)$ & -0.74 & $\left(-1.40_{-0.65}^{+0.66}, 7.04_{-1.77}^{+1.79}\right)$ \\
\hline & -0.35 & $5.2 \times 10^{-3}$ & -0.77 & $2.5 \times 10^{-2}$ \\
\hline \multirow[t]{2}{*}{$<L_{\gamma, \text { iso }}>_{45}-E_{\gamma, \text { peak }}$} & 0.81 & $\left(1.14_{-0.25}^{+0.22}, 49.27_{-0.60}^{+0.61}\right)$ & 0.76 & $\left(1.45_{-0.54}^{+0.26}, 48.48_{-1.04}^{+1.05}\right)$ \\
\hline & 0.67 & $2.6 \times 10^{-9}$ & 0.92 & $1.2 \times 10^{-3}$ \\
\hline
\end{tabular}

Table 8. Correlation coefficients $\rho$, the respective relation fit line parameters $(\mathrm{a}, \mathrm{b})$, and the correlation coefficient $r$ with the respective random occurrence probability $P$, for the considered prompt-afterglow and prompt-prompt distributions in log scale from Dainotti et al. (2011b).

In general, this study pointed out that the plateau phase results connected to the inner engine. In addition, also relations between $\log L_{X, a}$ and several other prompt emission parameters were analyzed, including $\log E_{\gamma, p e a k}$ and 
the variability, $\log V$. As a result, relevant relations are found between these quantities, except for the variability parameter, see Table 8 .
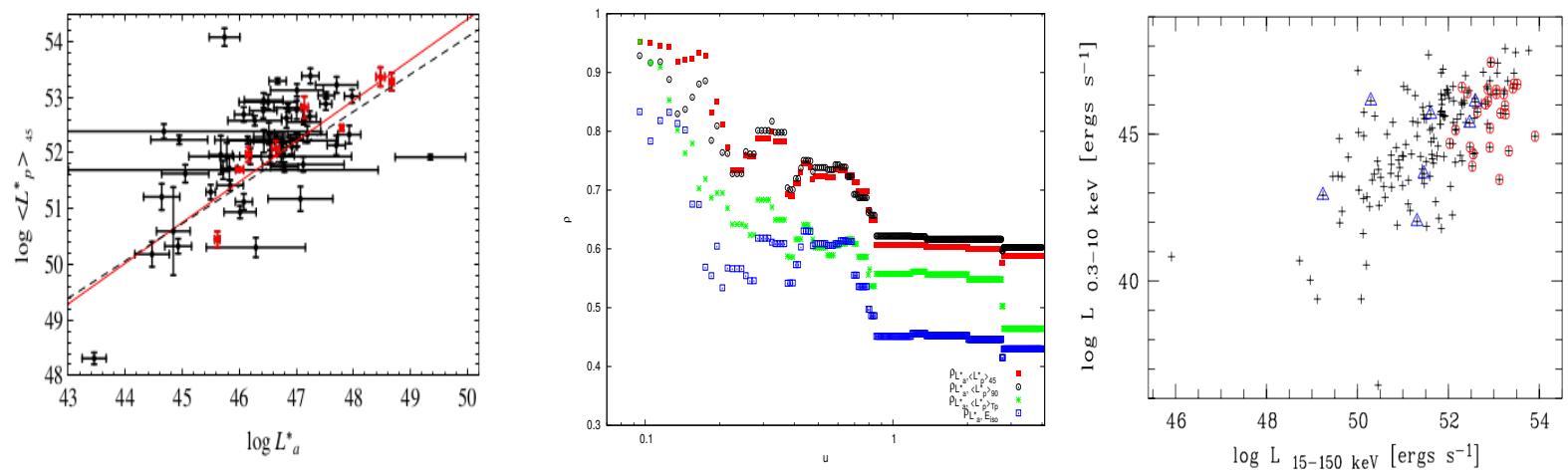

Figure 24. Left panel: $\log L_{X, a}$ vs. $\log <L_{\gamma, i s o}>_{45}$ relation $\left(\log L_{X, a}^{*}\right.$ and $\log L_{p}^{*}$ respectively) for the E4 data set from Dainotti et al. (2011b), with the fitted relation dashed line in black. The red line is fitted to the 8 lowest error (red) points of the E0095 subset. Middle panel: correlation coefficients $\rho$ for $\log L_{X, a}-\log <L_{\gamma, \text { iso }}>_{45}$ (red squares), $\log L_{X, a}-\log <L_{\gamma, \text { iso }}>_{90}$ (black circles), $\log L_{X, a}-\log \left\langle L_{\gamma, i s o}>_{T_{X, p}}\right.$ (green asterisks) and $\log L_{X, a}-\log E_{\gamma, \text { prompt }}$ (blue squares) relations, obtained using the LGRB subset with the maximum $\mathrm{u}=\sigma_{E}$ from Dainotti et al. (2011b). Right panel: Luminosity in the 0.3-10 keV XRT band ( $\left.L_{X, a}\right)$ vs. luminosity in the 15-150 keV BAT band $\left(L_{\gamma, \text { iso }}\right)$ from Grupe et al. (2013). Short bursts are represented by triangles and high-redshift $(\mathrm{z}>3.5)$ bursts by circles.

Finally, as shown in Table 8, only a very small relationship exists between $\log T_{X, a}^{*}-\log E_{\gamma, \text { prompt }}$ with $\rho=-0.19$. Also Grupe et al. (2013) claimed the existence of relations between $\log <L_{\gamma, i s o}>_{90}$ and $\log L_{X, a}$ (see the right panel of Fig. 24) and between $\log \left\langle L_{\gamma, i s o}>_{90}\right.$ and $\log T_{X, a}^{*}$ using a sample of 232 GRBs. The latter can be derived straightforwardly from the $\log T_{X, a}^{*}-\log E_{\gamma, \text { prompt }}$ relation, being $\log <L_{\gamma, \text { iso }}>_{90}$ computed as $\log \left(E_{\gamma, \text { prompt }} / T_{90}\right)$.

\subsection{The $L_{X, \text { peak }}-L_{X}\left(T_{a}\right)$ relation}

Dainotti et al. (2015b) further investigated the prompt-afterglow relations presenting an updated analysis of 123 Swift BAT+XRT light curves of LGRBs with known $z$ and afterglow plateau phase. The relation between the peak luminosity of the prompt phase in the X-ray, $\log L_{X, p e a k}$, and $\log L_{X, a}$ can be written as follows:

$$
\log L_{X, a}=A+B \times \log L_{X, p e a k},
$$

with $A=-14.67 \pm 3.46, B=1.21_{-0.13}^{+0.14}$ and with $\rho=0.79$ and $P<0.05$, see the left panel of Fig. 25 . In the literature $L_{X, p e a k}$ is denoted as:

$$
L_{X, p e a k}=4 \pi \times D_{L}\left(z, \Omega_{M}, h\right)^{2} \times F_{X, p e a k} \times K .
$$

The relation $<\log L_{\gamma, i s o}>-\log L_{X, a}$ (Dainotti et al. 2011b) for the same GRB sample presented a correlation coefficient, $\rho=0.60$, smaller than the one of the $\log L_{X, p e a k}-\log L_{X, a}$ relation, see sec. 4.4. This implied that a better definition of the luminosity or energy parameters improves $\rho$ by $24 \%$. In the left panel of Fig. $25 \log L_{X, p e a k}$ is calculated directly from the peak flux in X-ray, $F_{X, p e a k}$, considering a broken or a simple power law as the best fit of the spectral model. Thus, the error propagation due to time and energy is not involved, differently from the earlier considered luminosities. In addition, Dainotti et al. (2015b) preferred the $\log L_{X, p e a k}-\log L_{X, a}$ to the relations presented in Dainotti et al. (2011b), namely the $\left(\log E_{\gamma, \text { prompt }}, \log E_{\gamma, \text { peak }}\right)-\log L_{X, a}$, due to the fact that $\log E_{\gamma, \text { prompt }}$ and $\log E_{\gamma, \text { peak }}$ can undergo double bias truncation due to high and low energy detector threshold. Instead, this problem does not appear for $\log L_{X, p e a k}$ (Lloyd and Petrosian 1999). Furthermore, to show that the $\log L_{X, p e a k}-\log L_{X, a}$ relation is robust, the redshift dependence induced by the distance luminosity was eliminated employing fluxes rather than luminosities. A relation between $\log F_{X, a}$ and $\log F_{X, p e a k}$ was obtained with $\rho=0.63$, see the right panel of Fig. 25.

However, for further details about a quantitative analysis of the selection effects see sec. 5 .

Finally, Dainotti et al. (2015b) showed that the LT relation has a different slope, at more than $2 \sigma$, from the one of the 

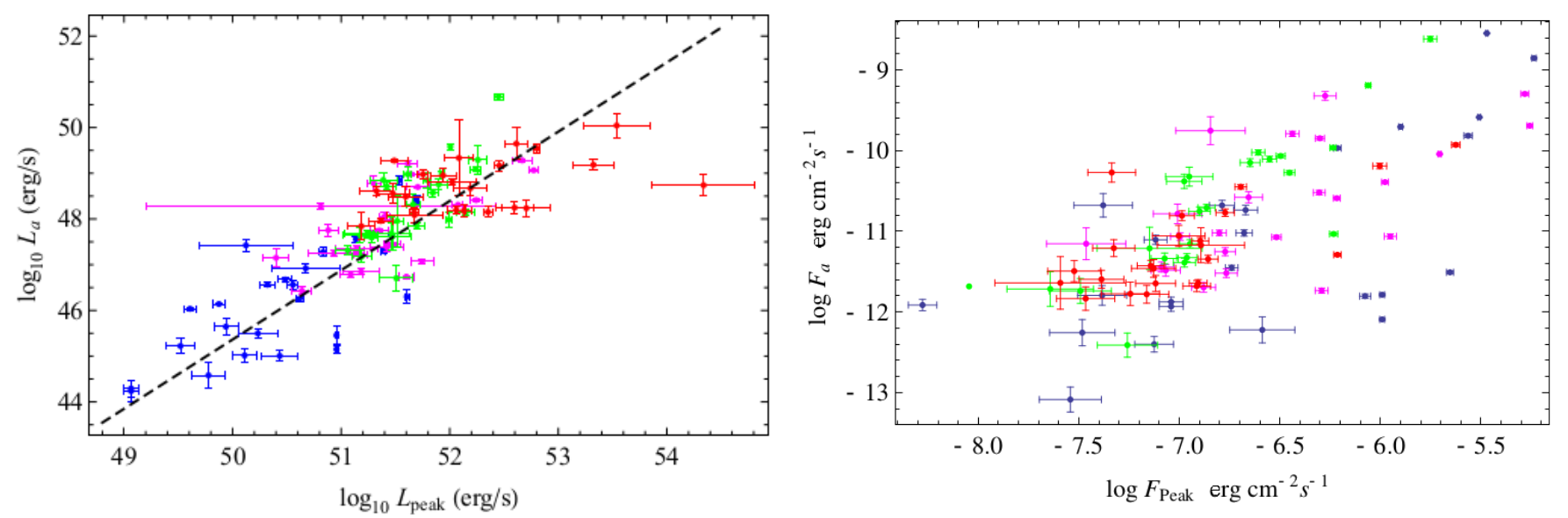

Figure 25. Left panel: "GRB distributions in redshift bins on the $\log L_{X, a}-\log L_{X, p e a k}$ plane from Dainotti et al. (2015b), where $\log L_{X, \text { peak }}$ is computed using the approach used in the Second BAT Catalogue. The sample is split into 4 different equipopulated redshift bins: $z \leq 0.84$ (blue), $0.84 \leq z<1.8$ (magenta), $1.8 \leq z<2.9$ (green) and $z \geq 2.9$ (red). The dashed line is the fitting relation line". Right panel: "GRB distributions in redshift bins on the $\log F_{X, a}-\log F_{X, p e a k}$ plane from Dainotti et al. (2015b), where $\log F_{X, p e a k}$ is computed following the approach used in the Second BAT Catalogue. The sample is split into 4 different equipopulated redshift bins: $z \leq 0.84$ (blue), $0.84 \leq z<1.8$ (magenta), $1.8 \leq z<2.9$ (green) and $z \geq 2.9$ (red)".
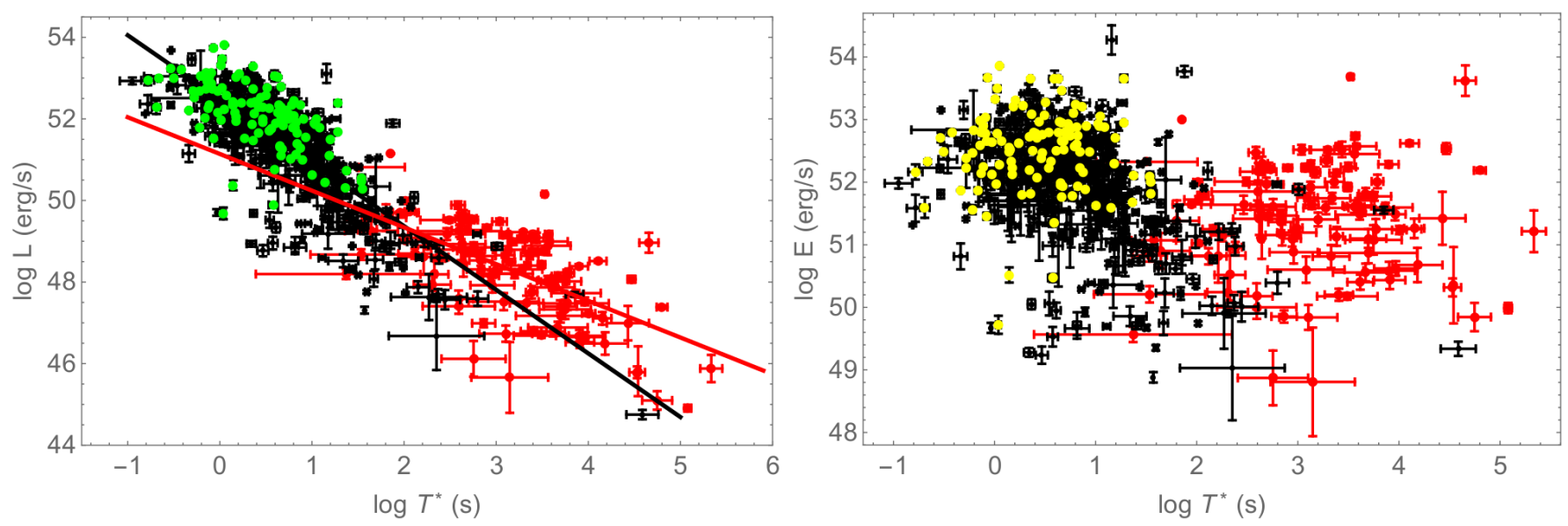

Figure 26. Left panel: $\log L-\log T^{*}$ relation for all the pulses in the prompt (black symbols) and in the afterglow (red symbols) emissions from Dainotti et al. (2015b). $\log L$ is the same as $\log L_{X, f}$ for the prompt emission pulses, while indicates $\log L_{X, a}$ for the afterglow emission pulses, and, the time $\log T^{*}$ indicates $\log T_{X, f}^{*}$ for the prompt emission pulses and $\log T_{X, a}^{*}$ for the afterglow phase. The green points show the maximum luminosity prompt emission pulses $\left(\log T_{L \max }, \log L_{\max }\right)$. Right panel: $\log E$ vs. $\log T^{*}$ relation for all the pulses in the prompt (black symbols) and in the afterglow (red symbols) emissions from Dainotti et al. (2015b). $\log E$ represents $\log E_{X, f}$ for the prompt emission pulses, while it represents $\log E_{X, \text { plateau }}$ for the afterglow emission pulses, and the time $\log T^{*}$ indicates $\log T_{X, f}^{*}$ for the prompt emission pulses and $\log T_{X, a}^{*}$ for the afterglow phase. The yellow points display the maximum energy prompt emission pulses $\left(\log T_{E \max }, \log E_{\max }\right)$.

prompt phase relation between the time since ejection of the pulse and the respective luminosity, $\log L_{X, f}-\log T_{X, f}^{*}$ (Willingale et al. 2010), see the left panel of Fig. 26. This difference also implied a discrepancy in the distributions of the energy and time, see the right panel of Fig. 26. The interpretation of this discrepancy between the slopes opens a new perspective in the theoretical understanding of these observational facts, see the next section for details.

As a further step, Dainotti et al. (2016a) analyzed this relation adding as a third parameter $T_{X, a}$ with a sample of 122 LGRBs (without XRFs and GRBs associated to SNe). They found a tight relation:

$$
\log L_{X, a}=(15.69 \pm 3.8)+(0.67 \pm 0.07) \times \log L_{X, p e a k}-(0.80 \pm 0.07) \log T_{X, a},
$$

with $\rho=0.93, P \leq 2.2 \times 10^{-16}$, and $\sigma_{\text {int }}=0.44 \pm 0.03$. Additionally, the scatter could be further reduced considering the subsample of 40 LGRBs having light curves with good data coverage and flat plateaus:

$$
\log L_{X, a}=(15.75 \pm 5.3)+(0.67 \pm 0.1) \times \log L_{X, p e a k}-(0.77 \pm 0.1) \log T_{X, a},
$$

with $\rho=0.90, P=4.41 \times 10^{-15}$, and $\sigma_{\text {int }}=0.27 \pm 0.04$. These results may suggest the use of this plane as a 
"fundamental" plane for GRBs and for further cosmological studies.

\subsubsection{Physical interpretation of the $L_{X}\left(T_{a}\right)-L_{\gamma, \text { iso }}$ and the $L_{X, p e a k}-L_{X}\left(T_{a}\right)$ relations}

In Dainotti et al. (2015b), the two distinct slopes of the luminosity-duration and the energy-duration distributions of prompt and plateau pulses could reveal that these two are different characteristics of the radiation: the former may be generated by internal shocks and the latter by the external shocks. Indeed, if the plateau is produced by synchrotron radiation from the external shock, then all the pulses have analogous physical conditions (e.g. the power law index of the electron distribution). In addition, the prompt-afterglow connections were analyzed in order to better explain the existing physical models of GRB emission predicting the $\log L_{X, a}-\log L_{\gamma, \text { iso }}$ and the $\log L_{X, p e a k}-\log L_{X, a}$ relations together with the LT one in the prompt and afterglow phases. They claimed that the model better explaining these relationships is the one by Hascoët et al. (2014). In this work they considered two scenarios: one in the standard FS model assuming a modification of the microphysics parameters to decrease the efficiency at initial stages of the GRB evolution; in the latter the early afterglow stems from a long-lived RS in the FS scenario. In the FS scenario a wind external medium is assumed together with a microphysics parameter $\epsilon_{e} \propto n^{-\nu}$, the amount of the internal energy going into electrons (or positrons), where $\mathrm{n}$ is the density medium. In the case of $\nu \approx 1$ is possible to reproduce a flat plateau. Thus, even operating on just one parameter can lead to the formation of a plateau that also reproduces the $\log L_{X, a}-\log L_{X, \text { iso }}$ and the $\log L_{X, p e a k}-\log L_{X, a}$ relations. Alternatively, in the RS scenario, in order to obtain the observed prompt-afterglow relationships, the typical $\Gamma$ of the ejecta should rise with the burst energy.

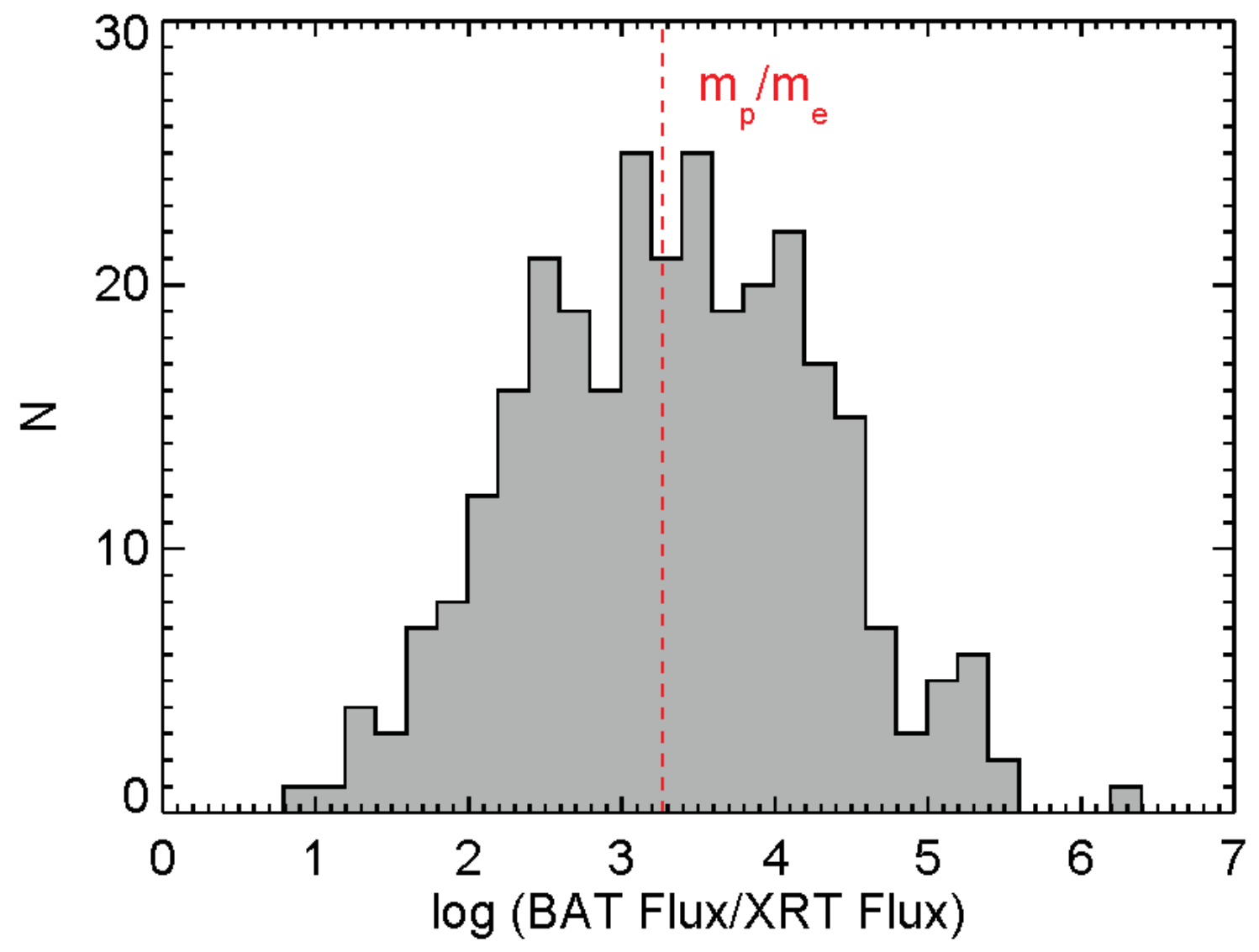

Figure 27. "The histogram of the BAT to XRT flux ratio for a number of Swift GRB from Kazanas et al. (2015). The distribution shows clearly a preferred value for this ratio of order $\sim 10^{3}-10^{4}$. The vertical line shows also the proton to electron mass ratio $m_{p} / m_{e}$ ".

In addition, Ruffini et al. (2014) pointed out that the induced gravitational collapse paradigm can recover the $\log L_{X, a}-\log L_{\gamma, \text { iso }}$ and the $\log L_{X, p e a k}-\log L_{X, a}$ relations. This model considers the very energetic $\left(10^{52}-10^{54} \mathrm{erg}\right)$ 
LGRBs for which the SNe has been seen. The light curves were built assuming for the external medium either a radial structure for the wind (Guida et al. 2008; Bernardini et al. 2006, 2007; Caito et al. 2009) or a partition of the shell (Dainotti et al. 2007), therefore well matching the afterglow plateau and the prompt emission.

Recently, Kazanas et al. (2015) within the context of the Supercritical Pile GRB Model claimed that they can reproduce the $\log L_{X, a}-\log L_{\gamma, i s o}$ and the $\log L_{X, p e a k}-\log L_{X, a}$ relations, because the ratio, $\mathrm{R}$, between the luminosities appears consistent with the one between the mean prompt energy flux from BAT and the afterglow plateau fluxes detected by XRT. In particular, $R \approx 2000$ is close to the proton to electron mass ratio, see Fig. 27.

Indeed, this is a new challenge for theoretical modelling that would need to consider, simultaneously, the several prompt-afterglow connections in order to better reproduce the phenomenology of the relations from a statistical point of view.

\subsection{The $L_{O, p e a k}^{F}-T_{O, p e a k}^{* F}$ relation and its physical interpretation}

Liang et al. (2010) studied the relation between the width of the light curve flares, $w$, and $T_{O, p e a k}$ of the flares, denoted with the index F, using a sample of 32 Swift GRBs, see the left panel of Fig. 28. This relation reads as follows:

$$
\log w^{F}=(0.05 \pm 0.27)+(1.16 \pm 0.10) \times \log T_{O, p e a k}^{F},
$$

with $\rho=0.94$.
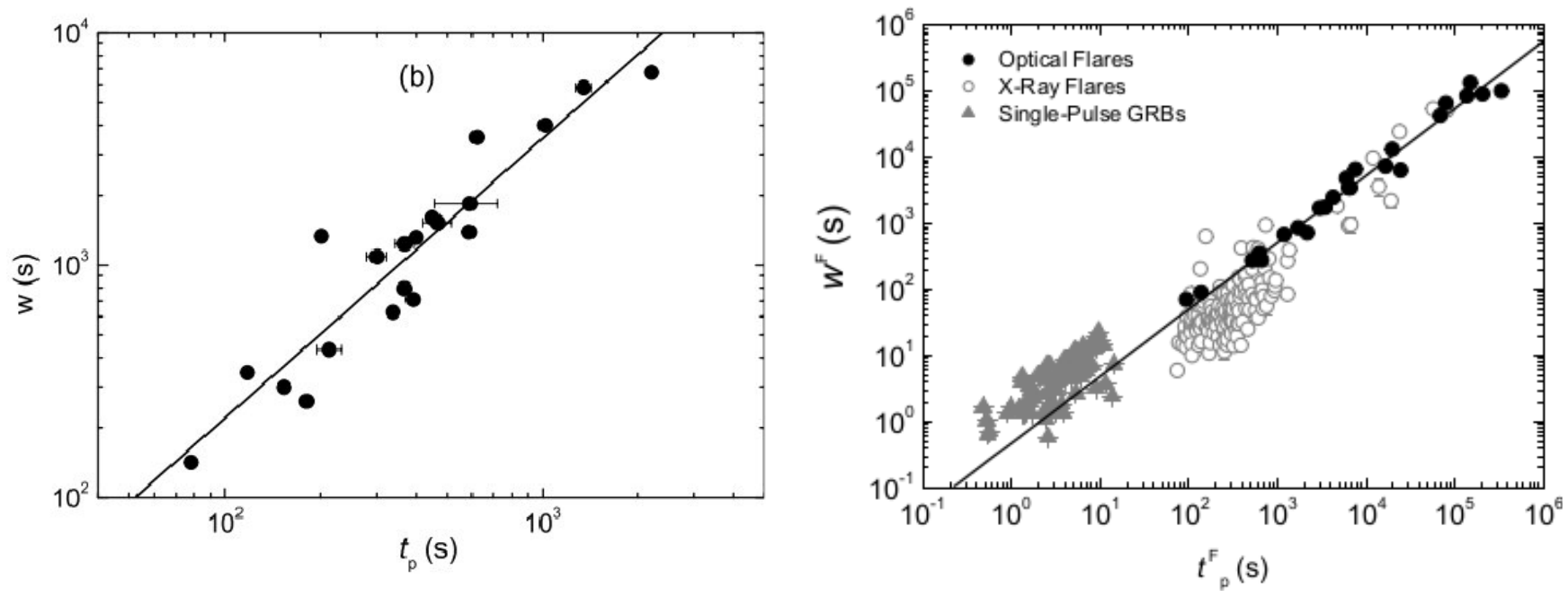

Figure 28. Left panel: $\log w^{F}-\log T_{O, p e a k}^{F}$ distribution from Liang et al. (2010). Right panel: $\log w^{F}-\log T_{O, p e a k}^{F}$ distribution from Li et al. (2012). In both panels lines represent the best fit.

Later, Li et al. (2012) found the same relation as Liang et al. (2010), but with smaller values of normalization and slope, using 24 flares from 19 single-pulse GRBs observed with CGRO/BATSE ${ }^{6}$, see the right panel of Fig. 28. However, for these 19 GRBs only in 14 GRBs a flare activity is distinctly visible. The relationship was given by:

$$
\log w^{F}=-0.32+1.01 \times \log T_{O, \text { peak }}^{F} .
$$

They claimed that earlier flares are brighter and narrower than later ones. They compared the $w^{F}-T_{O, \text { peak }}^{F}$ distribution for the X-ray flares detected by Swift/XRT with the one for the optical flares in the R band. As a conclusion, they seemed to have a similar behaviour (Chincarini et al. 2007; Margutti et al. 2010), see the right panel of Fig. 28.

Furthermore, in the rest frame band, they found a relation between the $L_{O, \text { peak }}$ of the flares in the $\mathrm{R}$ energy in units of $10^{48} \mathrm{erg} \mathrm{s}^{-1}$ and $T_{O, \text { peak }}^{*}$ of the flares using $19 \mathrm{GRBs}$, see Fig. 29. Both prompt pulses and X-ray and optical flares

${ }^{6}$ Among the instruments of the Compton Gamma Ray Observatory (CGRO) satellite, running from 1991 to 2001 , the Burst and Transient Source Experiment (BATSE) played a fundamental role in the measurements of GRB spectral features in the range from $20 \mathrm{keV}$ to $8 \mathrm{MeV}$. Bursts were typically detected at rates of roughly one per day over the 9-year CGRO mission within a time interval ranging from $\sim 0.1 \mathrm{~s}$ up to about $100 \mathrm{~s}$. Therefore, this satellite enabled careful analysis of the spectral properties of the GRB prompt emission. 
are correlated and present a visible temporal evolution, as seen in Fig. 29. This relation is given by:

$$
\log L_{O, \text { peak }}^{F}=(1.89 \pm 0.52)-(1.15 \pm 0.15) \times \log T_{O, p e a k}^{* F}
$$

with $\rho=0.85$ and $P<10^{-4}$. $T_{O, \text { peak }}^{F}$ spans from $\sim$ tens of seconds to $\sim 10^{6}$ seconds, instead the $L_{O \text {,peak }}^{F}$ varies from $10^{43}$ to $10^{49} \mathrm{erg} \mathrm{s}^{-1}$, with an average value of $10^{46} \mathrm{erg} \mathrm{s}^{-1}$. In addition, considering only the most luminous GRBs, they found that $T_{O, \text { peak }}^{* F}$ was strongly anti-correlated to $E_{\gamma, \text { prompt }}$ in the $1-10^{4} \mathrm{keV}$ energy band:

$$
\log T_{O, \text { peak }}^{* F}=(5.38 \pm 0.30)-(0.78 \pm 0.09) \times \log E_{\gamma, \text { prompt }} / 10^{50},
$$

with $\rho=0.92$. These outcomes revealed that the GRB flares in the optical wavelength with higher $E_{\gamma, \text { prompt }}$ peak earlier and are much more luminous. In Table 9 a summary of the relations described in this section is displayed.

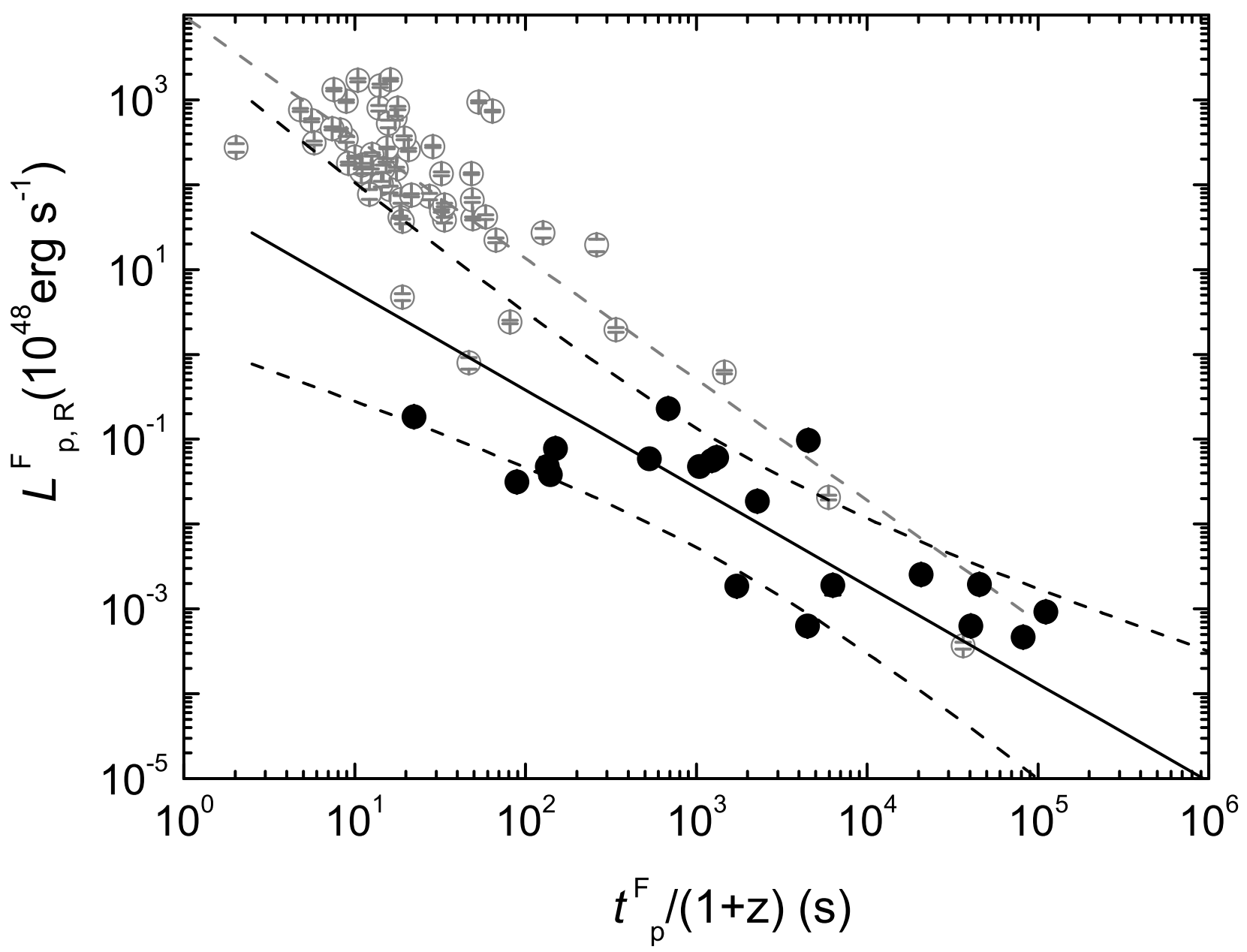

Figure 29. $\log L_{O, \text { peak }}^{F}-\log T_{O, \text { peak }}^{* F}$ relation from Li et al. (2012). Lines represent the best fits, black dots indicate optical flares, and the grey circles with errors show X-ray flares associated with the optical flares.

As regards the physical interpretation of the $L_{O, \text { peak }}^{F}-T_{O, p e a k}^{* F}$ relationship, Li et al. (2012) found that the flares are separated components superimposed to the afterglow phase. The coupling between $L_{O, \text { peak }}^{F}$ and $T_{O, \text { peak }}^{* F}$ suggested that the prompt $\gamma$-ray and late optical flare emission may arise from the same mechanism, namely from a central engine that can periodically eject a number of shells during the emission. Impacts of these shells could create internal shocks or magnetic turbulent reconnections, which would emerge from the variability (Kobayashi et al. 1997; Zhang and Yan 2011). Fenimore et al. (1995) revealed no relevant pattern in the width and intensity distributions using gamma ray data only. In addition, the usual tendency of the $w^{F}-T_{O, p e a k}^{F}$ relation cannot be due to hydrodynamical diffusion of the shells emitted at recent times, but it is necessary that the central engine radiates thicker and fainter shells at late stages (Maxham and Zhang 2009). This could be explained as flares generated by clumps, such that the diffusion 


\begin{tabular}{|c|c|c|c|c|c|c|}
\hline Correlations & Author & $\mathrm{N}$ & Slope & Norm & Corr.coeff. & $\mathrm{P}$ \\
\hline$w^{F}-T_{O, \text { peak }}^{F}$ & Liang et al. (2010) & 32 & $1.16_{-0.10}^{+0.10}$ & $0.05_{-0.27}^{+0.27}$ & 0.94 & $<10^{-4}$ \\
& Li et al. (2012) & 19 & 1.01 & -0.32 & & \\
\hline$L_{O, \text { peak }}^{F}-T_{O, \text { peak }}^{* F}$ & Li et al. (2012) & 19 & $-1.15_{-0.15}^{+0.15}$ & $1.89_{-0.52}^{+0.52}$ & 0.85 & $<10^{-4}$ \\
\hline$T_{O, \text { peak }}^{* F}-E_{\gamma, \text { prompt }}$ & Li et al. (2012) & 19 & $-0.78_{-0.09}^{+0.09}$ & $5.38_{-0.30}^{+0.30}$ & 0.92 & $<10^{-4}$ \\
\hline
\end{tabular}

Table 9. Summary of the relations in this section. The first column represents the relation in log scale, the second one the authors, the third one the number of GRBs in the used sample, and the fourth and the fifth columns are the slope and normalization of the relation. The last two columns are the correlation coefficient and the chance probability, $\mathrm{P}$.

during the accretion mechanism would extend the accretion duration onto the BH (Perna et al. 2006; Proga and Zhang 2006).

\section{SELECTION EFFECTS}

Selection effects are distortions or biases that usually occur when the sample observed is not representative of the "true" population itself. This kind of biases usually affects GRB relations. Efron and Petrosian (1992), Lloyd and Petrosian (1999), Dainotti et al. (2013a, 2015a) and Petrosian et al. (2013) emphasized that when dealing with a multivariate data set, it is imperative to determine first the true relations among the variables, not those introduced by the observational selection effects, before obtaining the individual distributions of the variables themselves. This study is needed for claiming the existence of the intrinsic relations. A relation can be called intrinsic only if it is carefully tested and corrected for these biases.

The selection effects present in the relations discussed above are mostly due to the dependence of the parameters on the redshift, like in the case of the time and the luminosity evolution, or due to the threshold of the detector used.

In this section, we describe several different methods to deal with selection biases.

In paragraph 5.1, we discuss the redshift induced relation through a qualitative method, while in 5.2 we present a more quantitative approach through the EP method. In 5.3, we describe how to obtain the intrinsic relations corrected by selection biases, and in 5.4 we report the selection effects for the optical and X-ray luminosities. Lastly, in 5.5 we show the evaluation of the intrinsic relation through Monte Carlo simulations.

\subsection{Redshift induced relations}

An important source of possible selection effects is the dependence of the variables on the redshift. To this end, Dainotti et al. (2011a) investigated the redshift evolution of the parameters of the LT relation, because a change of the relation slope has been observed when comparing several analyses (Dainotti et al. 2008, 2010). Namely, in the first paper, it was found $b=-0.74_{-0.19}^{+0.20}$ and in the latter $b=-1.06_{-0.28}^{+0.27}$. Therefore, it became crucial to understand the reason of this change, even if the two slopes are still comparable at the 1- $\sigma$ level. The distribution of the 62 LGRBs in the sample is not uniform within the range $\left(z_{\min }, z_{\max }\right)=(0.08,8.26)$ with few data points at large redshifts. Even if this sample is sparse, it was important to investigate whether the calibration coefficients $\left(a, b, \sigma_{i n t}\right)$ were in agreement within the error bars over this large redshift interval, see the left panel of Fig. 30.

For this reason, the data set was separated in three redshift bins with the same number of elements, $Z 1=(0.08,1.56)$, $Z 2=(1.71,3.08)$ and $Z 3=(3.21,8.26)$ presented as blue, green and red points respectively in the left panel of Fig. 30. The results are presented in Table 10.

\begin{tabular}{|c|c|c|c|c|}
\hline Id & $\rho$ & $\left(b, a, \sigma_{\text {int }}\right)_{b f}$ & $b_{\text {median }}$ & $\left(\sigma_{\text {int }}\right)_{\text {median }}$ \\
\hline Z1 & -0.69 & $(-1.20,51.04,0.98)$ & $-1.08_{-0.30}^{+0.27}$ & $1.01_{-0.16}^{+0.20}$ \\
Z2 & -0.83 & $(-0.90,50.82,0.43)$ & $-0.86_{-0.16}^{+0.18}$ & $0.45_{-0.08}^{+0.09}$ \\
Z3 & -0.63 & $(-0.61,50.14,0.26)$ & $-0.58_{-0.15}^{+0.14}$ & $0.26_{-0.06}^{+0.07}$ \\
\hline
\end{tabular}

Table 10. Results of the calibration procedure for GRBs divided in three equally populated redshift bins with $\left(z_{\min }, z_{\max }\right)=(0.08,1.56)$, $(1.71,3.08),(3.21,8.26)$ for bins Z1, Z2, Z3 from Dainotti et al. (2011a). The subscript $b f$ displays the best fit values, while the median subscript shows the median values.

The correlation coefficient $\rho$ was found quite high in each redshift bins, supporting the independence of the LT relation on $z$. The slopes $b$ for bins $Z 1$ and $Z 2$ are comparable within the $68 \%$ CL, while the slopes in bins $Z 1$ and $Z 3$ only within the $95 \%$ CL, see Table 10 . On the contrary, the normalization $a$ is comparable in all the bins. From this analysis, it is not possible to confirm that the LT relation is shallower for larger $z$ GRBs, due to the low number 

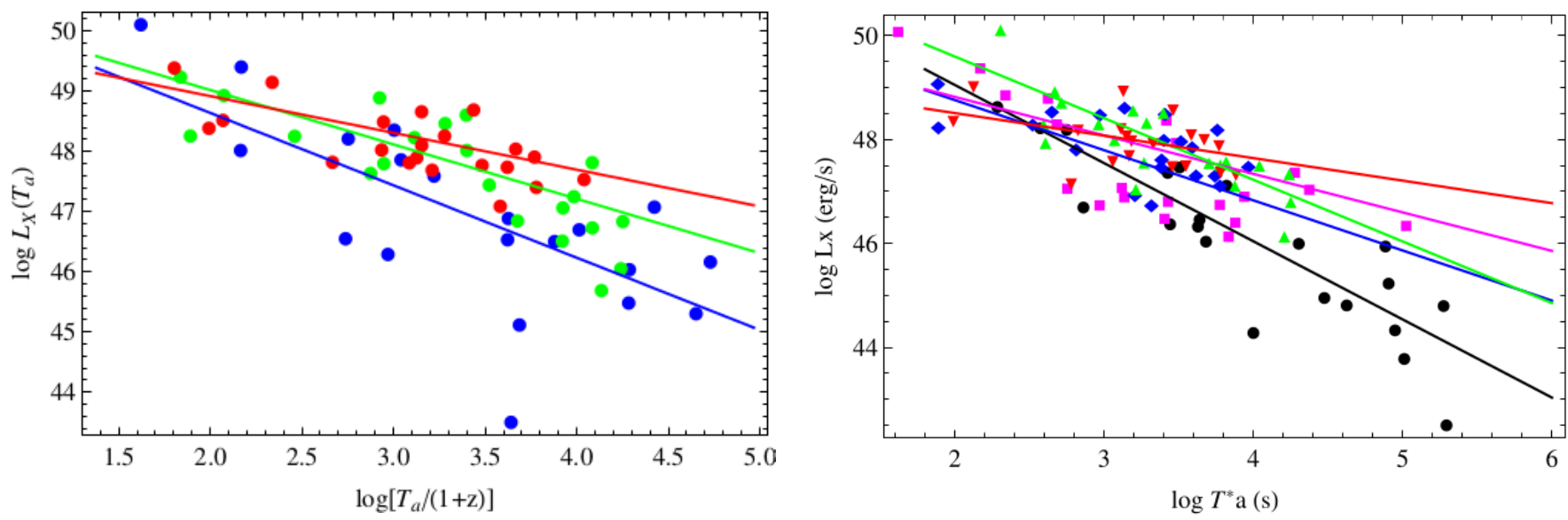

Figure 30. Left panel: " $\log L_{X, a}-\log T_{X, a}^{*}$ relation divided in the three redshift bins $Z 1=(0.08,1.56), Z 2=(1.71,3.08)$ and $Z 3=$ $(3.21,8.26)$ from Dainotti et al. (2011a). With the blue points it is represented the $Z 1$ sample, with the green ones the $Z 2$ sample and with the red points the $Z 3$ sample. The respective fitted lines are in the same colours". Right panel: "log $L_{X, a}-\log T_{X, a}^{*}$ distribution from Dainotti et al. (2013a) for the sample of 101 GRB afterglows divided in 5 equipopulated redshift bins shown by different colours: black for $z<0.89$, magenta for $0.89 \leq z \leq 1.68$, blue for $1.68<z \leq 2.45$, green $2.45<z \leq 3.45$, red for $z \geq 3.45$. Solid lines show the fitted relations".

of data points and the presence of high $\sigma_{E}$ GRBs. Finally, bigger samples with small $\sigma_{E}$ values and a more uniform $z$ binning are required to overcome this problem.

For this reason, Dainotti et al. (2013a) performed a similar analysis, but with a larger sample consisting of 101 GRBs. Specifically, this updated sample was split in 5 redshift ranges with the same number of elements, thus having 20 GRBs in each subgroup, represented in the right panel of Fig. 30 by different colours: black for $z<0.89$, magenta for $0.89 \leq z \leq 1.68$, blue for $1.68<z \leq 2.45$, green for $2.45<z \leq 3.45$ and red for $z \geq 3.45$. The fitted lines for each redshift bin are also shown in the same colour code. The distribution of the subsamples presented different power law slopes when the whole sample was divided into bins. The objects in the different bins exhibited some separation into different regions of the LT plane. Moreover, the slope of the relation for each redshift bin versus the averaged redshift range has also been presented, see the left panel of Fig. 31.

In addition, in Dainotti et al. (2015a), the updated sample of 176 GRBs was divided into 5 redshift bins consisting of about 35 GRBs for each group, as shown in the right panel of Fig. 31. A small evolution in $z$ has been confirmed with the following linear function $b(z)=0.10 z-1.38$.
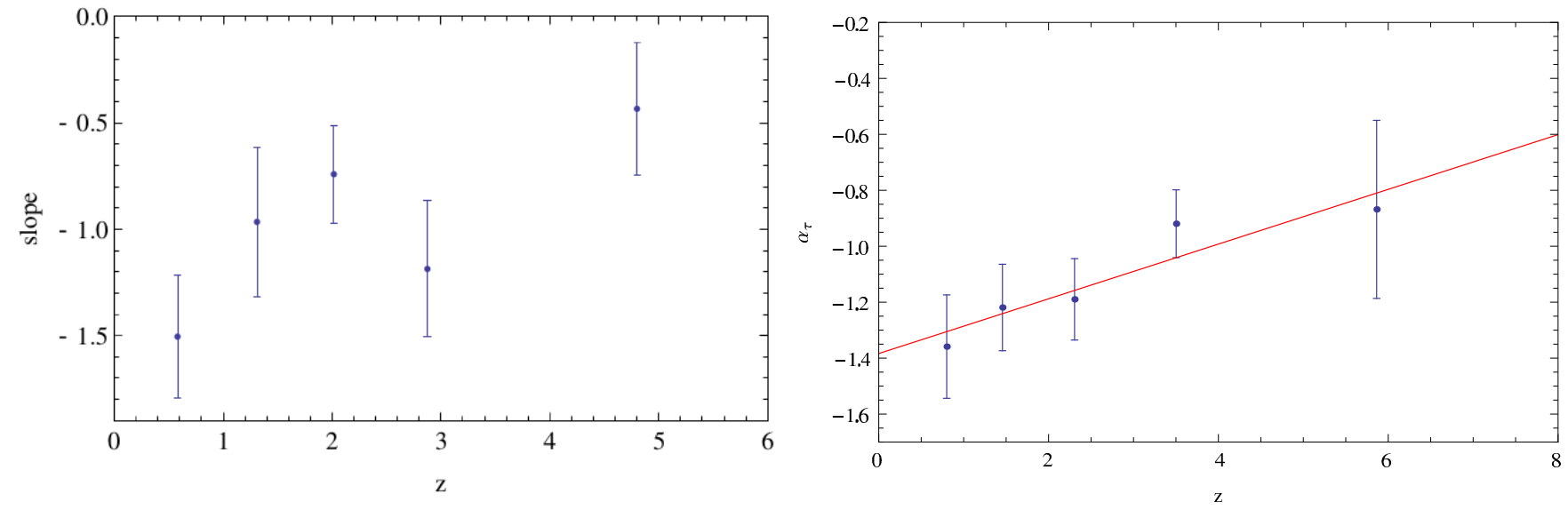

Figure 31. Left panel: "the variation of $b$ (and its error range) with the mean value of the redshift bins from Dainotti et al. (2013a)". Right panel: " $\alpha_{\tau}$, which is equivalent to the slope $b$, vs. z using a linear function $\alpha_{\tau}=0.10 z-1.38$ from Dainotti et al. (2015a)"

Regarding the $\log L_{X, p e a k}-\log L_{X, a}$ relation, Dainotti et al. (2015b) showed that it is not produced by the dependence on the redshift of its variables. To estimate the redshift evolution, the sample was separated into 4 redshift bins as shown in the left panel of Fig. 25. The GRB distribution in each bin is not grouped or constrained within a specific region, therefore indicating no strong redshift evolution. For $\log L_{X, a}$ it was found that there was negligible redshift 
evolution of the afterglow X-ray luminosity (Dainotti et al. 2013a), while for $\log L_{X, p e a k}$ has been demonstrated that there is significant redshift evolution (Yonetoku et al. 2004; Petrosian et al. 2013; Dainotti et al. 2015b). For more details, see sec. 5.2.1 and 5.2.2.

\subsection{Redshift induced relations through Efron and Petrosian method}

For a quantitative study of the redshift evolution, which is the dependence of the variables on the redshift, we here refer to the EP method which is specifically designed to overcome the biases resulting from incomplete data. The Efron \& Petrosian technique, applied to GRBs (Petrosian et al. 2009; Lloyd and Petrosian 1999; Lloyd et al. 2000), allows to compute the intrinsic slope of the relation by creating new bias-free observables, called local variables and denoted with the symbol '. For these quantities, the redshift evolution and the selection effects due to instrumental thresholds are removed. The EP method uses a modification of the Kendall tau test ${ }^{7} \tau$ to compute the best fit values of the parameters which represent the luminosity and time evolutionary functions. For details about the definition of $\tau$ see Efron and Petrosian (1992).

\subsubsection{Luminosity evolution}

The relation between luminosity and $z$ is called luminosity evolution. We discuss the luminosity evolution for both prompt and plateau phases. Before applying the EP method to the plateau phase, the limiting plateau flux, $F_{\text {lim }}$, which gives the minimum observed luminosity for a given $z$ needs to be parameterized. The XRT sensitivity, $F_{\text {lim,XRT }}=10^{-14} \mathrm{erg}^{-2} \mathrm{~cm}^{-1}$, is not high enough to represent the truncation of the data set. Hence, as claimed by Cannizzo et al. (2011), a better choice for the flux threshold is $10^{-12} \mathrm{erg} \mathrm{cm}^{-2} \mathrm{~s}^{-1}$. Several threshold fluxes were analyzed (Dainotti et al. 2013a), finally $F_{l i m, X R T}=1.5 \times 10^{-12} \mathrm{erg} \mathrm{cm}^{-2} \mathrm{~s}^{-1}$, which leaves 90 out of 101 GRBs, was selected (see the left panel of Fig. 32). Regarding instead the prompt limiting flux, Dainotti et al. (2015b) chose a BAT flux limit $F_{\text {lim, } B A T}=4 \times 10^{-8} \mathrm{erg} \mathrm{cm}^{-2} \mathrm{~s}^{-1}$, which also allows $90 \%$ of GRBs in the sample, see the right panel of Fig. 32.
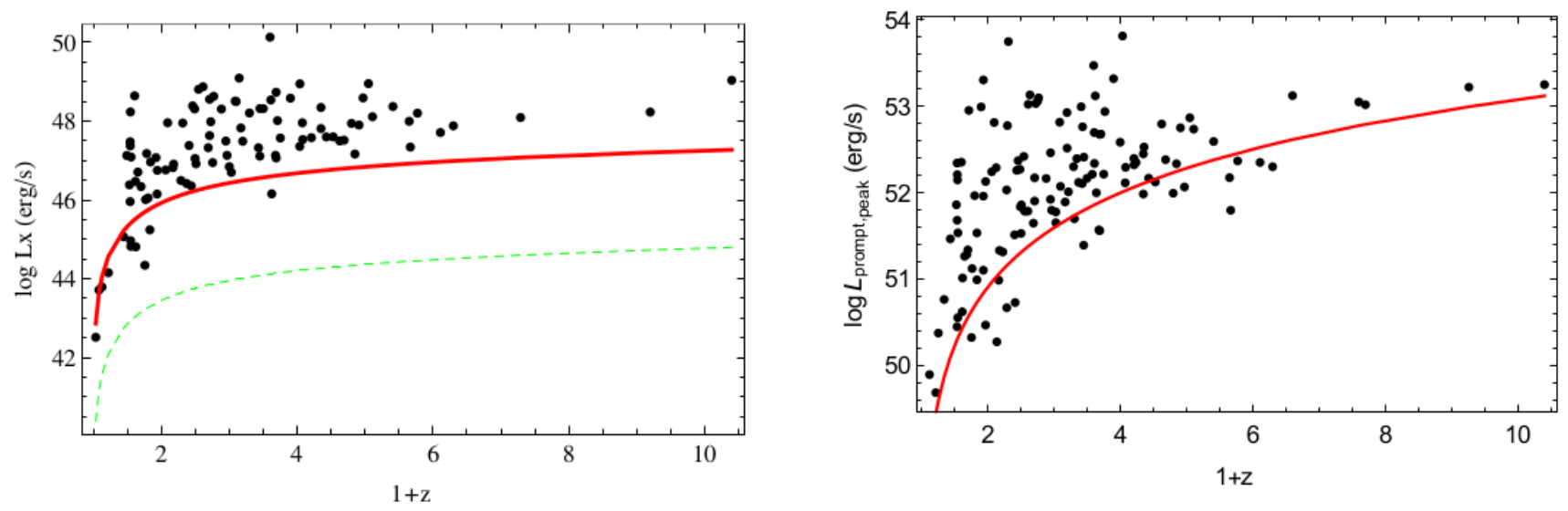

Figure 32. Left panel: "the bivariate distribution of $\log L_{X, a}$ and $z$ with two different flux limits from Dainotti et al. (2013a). The instrumental XRT flux limit, $1.0 \times 10^{-14} \mathrm{erg} \mathrm{cm}^{-2} \mathrm{~s}^{-1}$ (dashed green line), is too low to be representative of the flux limit, $1.5 \times 10^{-12}$ erg $\mathrm{cm}^{-2} \mathrm{~s}^{-1}$ (solid red line) represents better the limit of the sample". Right panel: "the bivariate distribution of $\log L_{X, p e a k}$ and $z$ with the flux limit assuming the $\mathrm{K}$ correction $K=1$ from Dainotti et al. (2015b). The BAT flux limit, $4.0 \times 10^{-8} \mathrm{erg} \mathrm{cm}^{-2} \mathrm{~s}^{-1}$ (solid red line), better represents the limit of the sample".

In Dainotti et al. (2013a), the relation function, $\mathrm{g}(\mathrm{z})$, is defined when determining the evolution of $L_{X, a}$ so that the local variable $L_{X, a}^{\prime} \equiv L_{X, a} / g(z)$ is not dependent anymore from $z$. The evolutionary function is parameterized by a simple relation function:

$$
g(z)=(1+z)^{k_{L_{X, a}}} .
$$

More complex evolution functions lead to comparable results, see Dainotti et al. (2013a, 2015b).

\footnotetext{
7 The Kendall $\tau$ is a non-parametric statistical test used to measure the association between two measured quantities. It is a measure of rank relation: the similarity of the orderings of the data when ranked by each of the quantities.
} 

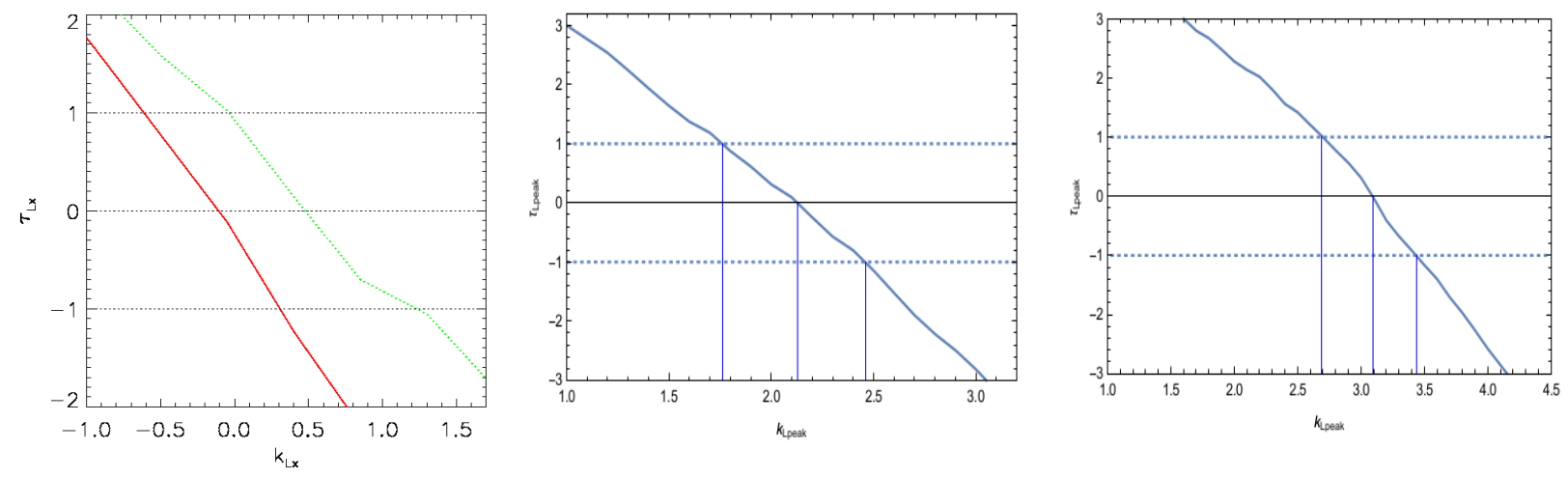

Figure 33. Left panel: $\tau$ vs. $k_{L_{X, a}}$ from Dainotti et al. (2013a). The red line indicates the full sample, while the green dotted line indicates the sample of 47 GRBs in common with the 77 LGRBs in Dainotti et al. (2011a). Middle panel: $\tau$ vs. $k_{L_{X}, p e a k}$, using the eq. 20, from Dainotti et al. (2015b). Right panel: $\tau$ vs. $k_{L_{X, p e a k}}$, using the eq. 21, from Dainotti et al. (2015b).

With this modified version of $\tau$, the value of $k_{L_{X, a}}$ for which $\tau_{L_{X, a}}=0$ is the one that best represents the luminosity evolution at the $1 \sigma$ level. $k_{L_{X, a}}=-0.05_{-0.55}^{+0.35}$ means that this evolution is negligible, see the left panel of Fig. 33. In the same panel, this distribution is also plotted for a smaller sample of 47 GRBs (green dotted line) in common with the previous one of 77 LGRBs presented in Dainotti et al. (2011a).

The results of the afterglow luminosity evolution among the two samples are compatible at $2 \sigma$. Instead, regarding the study of the evolution of $L_{X, p e a k}$, the simple relation function (see eq. 20) was compared to a more complex function (Dainotti et al. 2015b) given by:

$$
g(z)=\frac{Z^{k_{L}}\left(1+Z_{c r}^{k_{L}}\right)}{Z^{k_{L}}+Z_{c r}^{k_{L}}}
$$

where $Z=1+z$ and $Z_{c r}=3.5$. A relevant luminosity evolution was obtained in the prompt, $k_{L_{X, p e a k}}=2.13_{-0.37}^{+0.33}$, using the simple relation, while $k_{L_{X, p e a k}}=3.09_{-0.35}^{+0.40}$ for the more complex function, see the middle and right panels of Fig. 33 respectively. The results of the prompt luminosity evolution among the two different functions are compatible at $2 \sigma$.

\subsubsection{Time Evolution}

Similarly to the treatment of the luminosity evolution, one has also to determine the limit of the plateau end time, $T_{X, a, \text { lim }}^{*}=242 /(1+z) \mathrm{s}$ (Dainotti et al. 2013a), and of the prompt peak time $T_{X, \text { prompt,lim }}^{*}=1.74 /(1+z) \mathrm{s}$ (Dainotti et al. 2015b), see the left and right panels of Fig. 34 and Fig. 35 respectively.

To determine the evolution of $T_{X, a}^{*}$, so that the de-evolved variable $T_{X, a}^{\prime} \equiv T_{X, a}^{*} / f(z)$ is not correlated with z, the relation function $f(z)$ (Dainotti et al. 2013a) was specified:

$$
f(z)=(1+z)^{k_{T_{X}^{*}, a}^{*}} .
$$

The values of $k_{T_{X, a}^{*}}$ for which $\tau_{T_{X, a}^{*}}=0$ is the one that best matches the plateau end time evolution at the $1 \sigma$ uncertainty. $\tau_{T_{X, a}^{*}}$ versus $k_{T_{X, a}^{*}}$ distribution shows a consistent evolution in $T_{X, a}^{*}$, as seen in the left panel of Fig. 36, namely $k_{T_{X, a}^{*}}=-0.85_{-0.30}^{+0.30}$. In the same panel this distribution is also displayed for a smaller sample of 47 GRBs (green dotted line) in common with the previous one of 77 GRBs presented in Dainotti et al. (2011a). The results of the afterglow time evolution among the two samples are compatible at $1.5 \sigma$.

Regarding the prompt time evolution, a more complex function was also used in addition to the simple relation function (Dainotti et al. 2015b):

$$
f(z)=\frac{Z^{k_{T}^{*}}\left(1+Z_{c r}^{k_{T}^{*}}\right)}{Z^{k_{T}^{*}}+Z_{c r}^{k_{T}^{*}}}
$$

where $Z=1+z$ and $Z_{c r}=3.5$.

As a conclusion, a not relevant time evolution in the prompt was found for both the simple function, $k_{T_{X, p r o m p t}^{*}}=$ $-0.62_{-0.38}^{+0.38}$, and for the more complex one $k_{T_{X, p r o m p t}^{*}}=-0.17_{-0.27}^{+0.24}$, see the middle and right panels of Fig. 36 respectively. The results of the prompt time evolution among the two different functions are compatible at $1 \sigma$. 

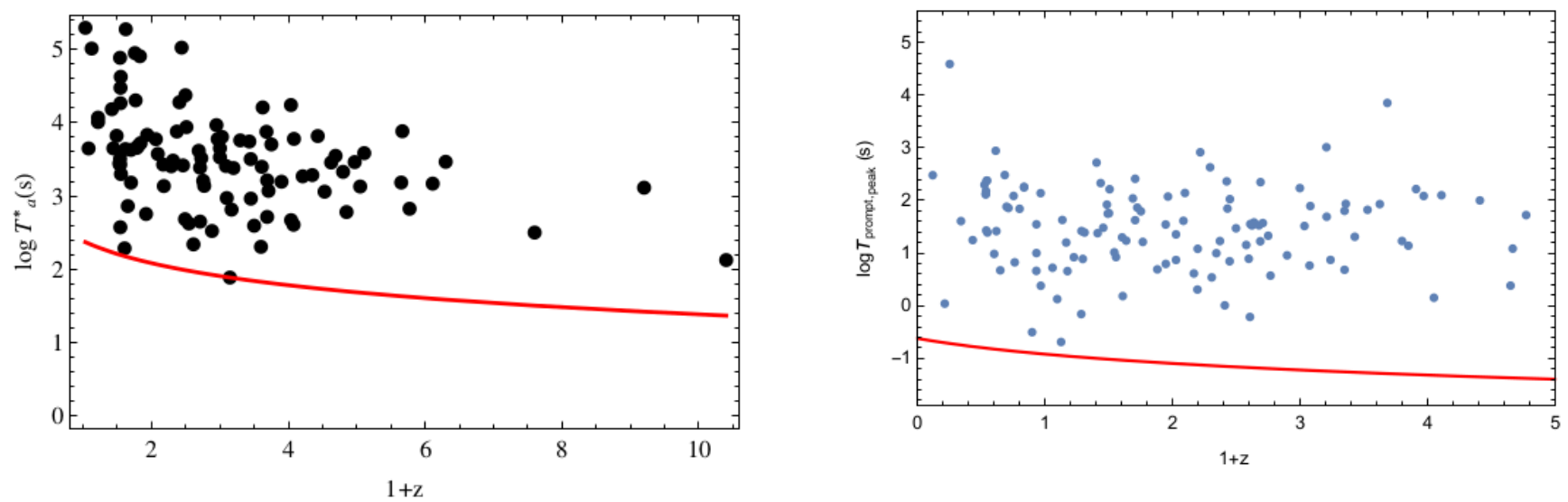

Figure 34. Left panel: "the bivariate distribution of the rest frame time $\log T_{X, a}^{*}$ and $z$ from Dainotti et al. (2013a). The red line is the limiting rest frame time, $\log \left(T_{X, a, l i m} /(1+z)\right)$ where the chosen limiting value of the observed end-time of the plateau in the sample is $T_{X, a, l i m}=242 \mathrm{~s}$ ". Right panel: "the bivariate distribution of the rest frame time $\log T_{X, p r o m p t}^{*}$ and $z$ from Dainotti et al. (2015b), where with $\log T_{X}^{*}$,prompt they denoted the sum of the peak pulses width of each single pulse in each GRB. The chosen limiting value of the observed pulse width in the sample is $\log T_{X, \text { prompt,lim }}=0.24 \mathrm{~s}$. The red line is the limiting rest frame time, $\log \left(T_{X, p r o m p t, l i m} /(1+z)\right)$.
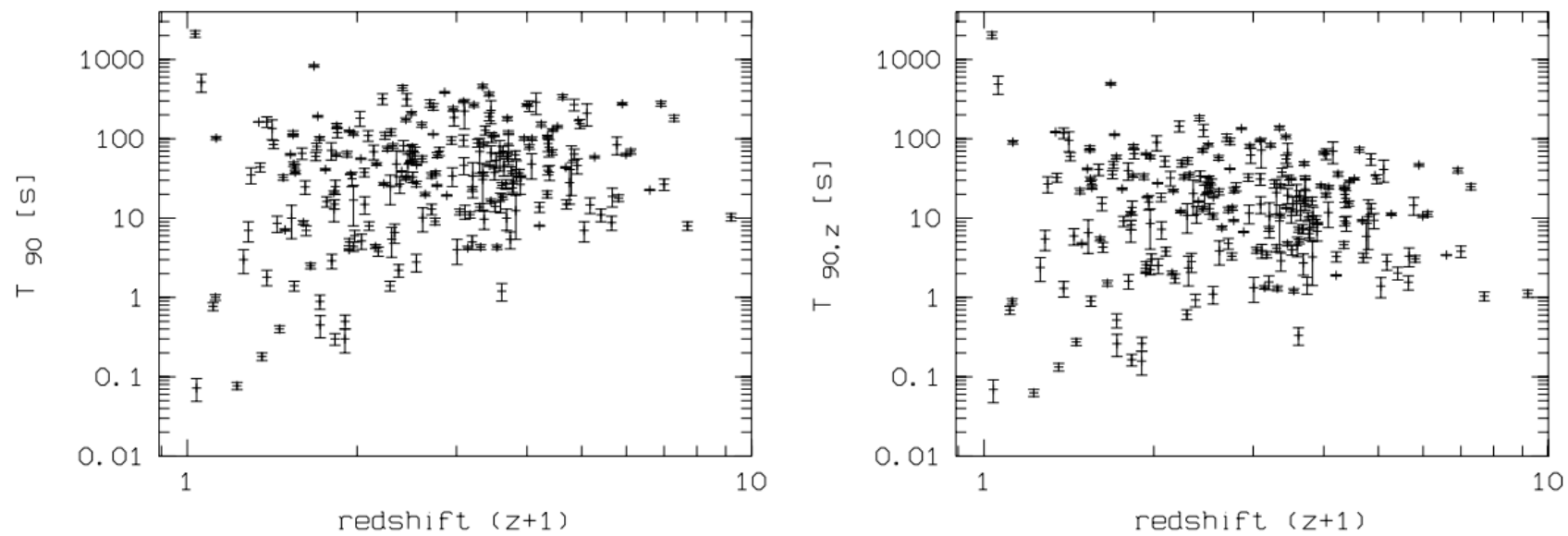

Figure 35. Distributions between redshift and the observed (left panel) and rest-frame (right panel) $T_{90}$ in the BAT energy range from Grupe et al. (2013).
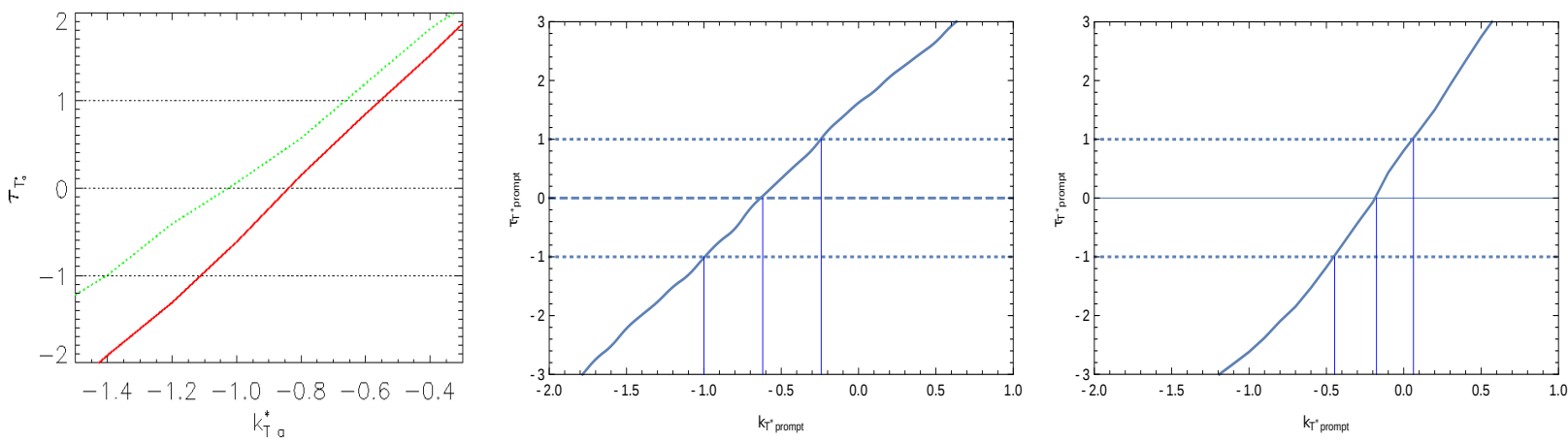

Figure 36. Left panel: $\tau$ vs. $k_{T_{X, a}^{*}}$ from Dainotti et al. (2013a). The red line indicates the full sample, while the green dotted line indicates the 47 GRBs in common with the sample presented in Dainotti et al. (2011a). Middle panel: $\tau$ vs. $k_{T_{X, p r o m p t}^{*}}$, using the eq. 22 , from Dainotti et al. (2015b). Right panel: $\tau$ vs. $k_{T_{X, p r o m p t}^{*}}$, using the eq. 23, from Dainotti et al. (2015b). 


\subsection{Evaluation of the intrinsic slope}

The last step to determine if a relation is intrinsic is to evaluate its "true" slope. To this end, the EP method was used in the local time $\left(T_{X, a}^{\prime}\right)$ and luminosity $\left(L_{X, a}^{\prime}\right)$ space obtaining an intrinsic slope for the LT relation $b_{i n t}=1 / \alpha=$ $-1.07_{-0.14}^{+0.09}$. The significance of this relation is at $12 \sigma$ level. It can be derived directly from the left panel of Fig. 37 (Dainotti et al. 2013a), because if there was no relation it would have been that $\tau=0$ for $b_{\text {int }}=0$ at $1 \sigma$.

Instead, regarding the evaluation of the intrinsic slope in the $\log L_{X, p e a k}-\log L_{X, a}$ relation, Dainotti et al. (2015b) used a different method, namely the partial correlation coefficient. This is the degree of association between two random variables calculated as a function of $b_{\text {int }}$ in the following way:

$$
r_{L_{X, p e a k}^{\prime} L_{X, a}^{\prime}, D_{L}}=\frac{r_{L_{X, p e a k}^{\prime}, L_{X, a}^{\prime}}-r_{L_{X, p e a k}^{\prime}, D_{L}} * r_{L_{X, a}^{\prime}, D_{L}}}{\left(1-r_{L_{X, p e a k}^{\prime}, D_{L}}^{2}\right) *\left(1-r_{L_{X, a}^{\prime}, D_{L}}^{2}\right)}
$$

where $\log L_{X, a}^{\prime}=L_{X, a}^{\prime}$ and $\log L_{X, p e a k}^{\prime}=L_{X, p e a k}^{\prime}$.
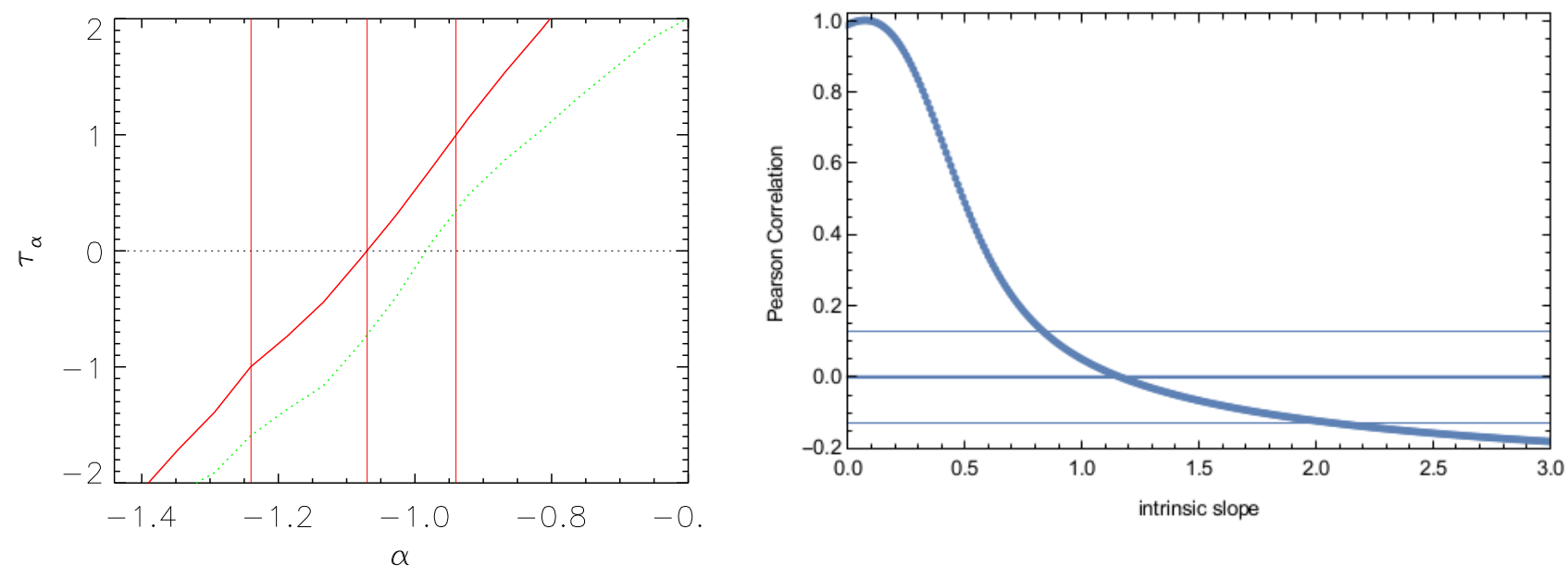

Figure 37. Left panel: $\tau$ vs. $b_{\text {int }}$ (indicated with $\alpha$ in the picture) from Dainotti et al. (2013a). Right panel: $\mathrm{r}$ vs. $b_{i n t}$ from Dainotti et al. (2015b) with the best value where $\log L_{X, p e a k}$ and $\log L_{X, a}$ are strongly correlated (the central thick line). The two thinner lines indicate the $0.05 \%$ probability that the sample is drawn by chance.

As displayed in the right panel of Fig. 37, the relation is highly significant when $b_{\text {int }}=1.14_{-0.32}^{+0.83}$, which is at $1 \sigma$ of the observed slope.

In addition, following an analysis similar to the one of Butler et al. (2010), Dainotti et al. (2015a) simulated a sample for which biases on both time and luminosity are considered. Particularly, they assumed the biases to be roughly the same whichever monotonic efficiency function for the luminosity detection is taken. This method presented how an unknown efficiency function could affect the slope of any relation and the GRB density rate. Then, biases in slope or normalization caused by the truncations were analyzed. This gave distinct fit values that allow for studying the scatter of the relation and its selection effects. This analysis has shown, together with the one in Dainotti et al. (2013a), that the LT relation can be corrected by selection effects and therefore can be used in principle as redshift estimator (see sec. 6) and as a valuable cosmological tool (see sec. 7). As regards other relations, D'Avanzo et al. (2012) for the $L_{X, a}-E_{\gamma, \text { prompt }}$ relation, Oates et al. (2015) for the $L_{O, 200 \mathrm{~s}}-\alpha_{O,>200 \mathrm{~s}}$ relation, and Racusin et al. (2016) for the $L_{X, 200 \mathrm{~s}}-\alpha_{X,>200 \text { s }}$ relation, also used the partial correlation coefficient method to show that the redshift dependence does not induce these relations.

\subsection{Selection effects for the optical and X-ray luminosities}

In this section we discuss the selection effects due to the limiting optical and X-ray luminosities relevant for the relations mentioned above. Nardini et al. (2008b) investigated if the observed luminosity distribution can be the result of selection effects by studying the optically dark afterglows. By simulating the $\log L_{O, 12}, z$, host galaxy dust absorption, $A_{V}^{\text {host}}$, and telescope limiting magnitude for each of the $30000 \mathrm{GRBs}$, the observed optical luminosity distribution was contrasted to the simulated one. From this simulated distribution regarding the intrinsic one, it is 
necessary to take only GRBs with a flux which is larger than the threshold flux of the associated detector. This corresponds with a lower luminosity truncation, which is around $\log L_{O, 12} \approx 31.2\left(\mathrm{erg} \mathrm{s}^{-1} \mathrm{~Hz}^{-1}\right)$. Therefore, the fact that we do not observe GRBs with such a luminosity puts a limit to the luminosity function.

They also checked statistically the presence of a low luminosity category of events which are at $3.6 \sigma$ off the central value of the distribution. They pointed out that if the absorption is chromatic, the observed luminosity distribution does not match with any unimodal one. If many GRBs are absorbed by "grey" achromatic dust, then a unimodal luminosity distribution can be obtained. In summary, dark bursts could belong to an optically subluminous group or to a category of bursts for which a high achromatic absorption is present.

As regards the evaluation of the selection effects of $L_{O, \text { peak }}$, the biases in the detection of $F_{O, \text { peak }}$ need to be considered. As found from Panaitescu and Vestrand (2008), for a typical optical afterglow spectrum $\left(F_{O, a} \propto T_{O, a}^{-1}\right)$, variations in the observer offset angle induce a $\log F_{O, p e a k}-\log T_{O, p e a k}$ anti-relation that is flatter than what is measured. In fact, an observational selection effect could steepen the slope of the anti-relation between $\log F_{O, p e a k}$ and $\log T_{O, p e a k}$.

In addition, SGRBs observed by Swift seem to be fluence-limited, while LGRBs detected with the same telescope are flux-limited (Gehrels et al. 2008) due to the instrument trigger.

Nysewander et al. (2009) pointed out that the ratio $F_{O, 11} / F_{X, 11}$ may be influenced by absorption of photons in the host galaxy. Furthermore, they showed that $F_{X, 11}$ should be precise, because the LGRBs observed in the XRT passband do not present X-ray column absorptions, differently from the majority of LGRBs. The computed optical absorption of LGRB afterglows indicates smaller column densities $\left(N_{H}\right)$ than in the X-ray, with optical absorptions $\left(A_{V}\right)$ about one-tenth to one magnitude (Schady et al. 2007; Cenko et al. 2009). Regarding the SGRBs, they have more luminous optical emission relative to the X-ray than what is assumed by the standard model. Later, Kann et al. (2010) claimed that the grouping of the optical luminosity at the time of 1 day, $L_{O, 1 \mathrm{~d}}$, is less remarkable than the one described by Liang and Zhang (2006b) and Nardini et al. (2006) for GRBs observed by Swift. This suggested that the grouping pointed out in pre-Swift data can be due to selection effects only. Finally, Berger (2014) claimed that the optical afterglow detection can influence the luminosity distribution towards places with larger densities medium.

\subsection{Selection effects in the $L_{O, 200 \mathrm{~s}}-\alpha_{O,>200 \mathrm{~s}}$ relation}

Oates et al. (2012) ensured that a high S/N light curve, covering both early and late times, can be constructed from the UVOT multi-filter observations using the criteria from Oates et al. (2009). If the faintest optical/UV afterglows decay more slowly than the brightest ones, then at late time the luminosity distribution is less dispersed and the correlation coefficient of the $\log L_{O, 200 \mathrm{~s}}-\alpha_{O,>200 \mathrm{~s}}$ relation must become smaller and/or negligible. Indeed, both of these effects were observed in their sample. Furthermore, the $\log L_{O, 200 \mathrm{~s}}-\alpha_{O,>200 \mathrm{~s}}$ relation may arise, by chance, from the way in which the sample is chosen. Thus, to verify if this is not the case, they computed Monte Carlo simulations. Among the $10^{6}$ trials, 34 have a correlation coefficient indicating a more significant relation than the original one. This points out that, at $4.2 \sigma$ confidence, the $\log L_{O, 200 \mathrm{~s}}-\alpha_{O,>200 \mathrm{~s}}$ relation is not caused by the selection criteria nor does it happens by chance, and thus it is intrinsic.

\section{REDSHIFT ESTIMATOR}

As we have pointed out in the introduction, the study of GRBs as possible distance estimators is relevant, since for many of them $z$ is unknown. Therefore, having a relation which is able to infer the distance from known quantities observed independently of $z$ would allow a better investigation of the GRB population. Moreover, in the cases in which $z$ is uncertain, the estimator can give hints on the upper and lower limits of the distance at which the GRB is placed. Some examples of redshift estimators for the prompt relations (Atteia 2003; Yonetoku et al. 2004; Tsutsui et al. 2013) have been reported. In these papers, a method is developed for inverting GRB luminosity relations in respect to the redshift to have an expression of the distance as a function of $\mathrm{z}$. The methodology used for the prompt emission relations can be then applied also to the afterglow or prompt-afterglow phase relations.

In this respect, Dainotti et al. (2011a) investigated the LT relation as a redshift estimator. From this relation, the best fit parameters of the slope and normalization are derived, while parameters such as $\log F_{X, a}, \log T_{X, a}$ and $\beta_{X, a}$ are known, because they are measured. Therefore, the LT relation can be inverted to obtain an estimate of $z$ as it has been done for the prompt relations by Yonetoku et al. (2004). With this intention, let us return to the eq. 2 and write it in another form: 


$$
\begin{aligned}
\log L_{X, a} & =\log \left(4 \pi F_{X, a}\right)+2 \log D_{L}\left(z, \Omega_{M}, h\right)-\left(1-\beta_{X, a}\right) \log (1+z) \\
& =\log \left(4 \pi F_{X, a}\right)+\left(1+\beta_{X, a}\right) \log (1+z)+2 \log r(z)+2 \log \left(c / H_{0}\right) \\
& =a \log \left(\frac{T_{X, a}}{1+z}\right)+b
\end{aligned}
$$

where $r(z)=D_{L}\left(z, \Omega_{M}, h\right) \times\left(H_{0} / c\right)$. Solving respect to $z$, it was obtained:

$$
\left(1+\beta_{X, a}+a\right) \log (1+z)+2 \log r(z)=a \log T_{X, a}+b-\log \left(4 \pi F_{X, a}\right)-2 \log \left(c / H_{0}\right) .
$$

The numerical solution of this equation may encounter some problems that must be taken into account: $\left(\log T_{X, a}, \log \right.$ $\left.F_{X, a}, \beta_{X, a}\right)$ and the LT calibration parameters $(a, b)$ are influenced by their own errors. Furthermore, the errors on $(a, b)$ are not symmetric and $\sigma_{i n t}$ is summed to the total error in a nonlinear way. For details about possible solutions on how to consider the errors see Dainotti et al. (2011a). The above solution was employed for the E4 and the E0095 samples, pointing out that the LT relation can still not be considered as a precise redshift estimator, see Fig. 38.

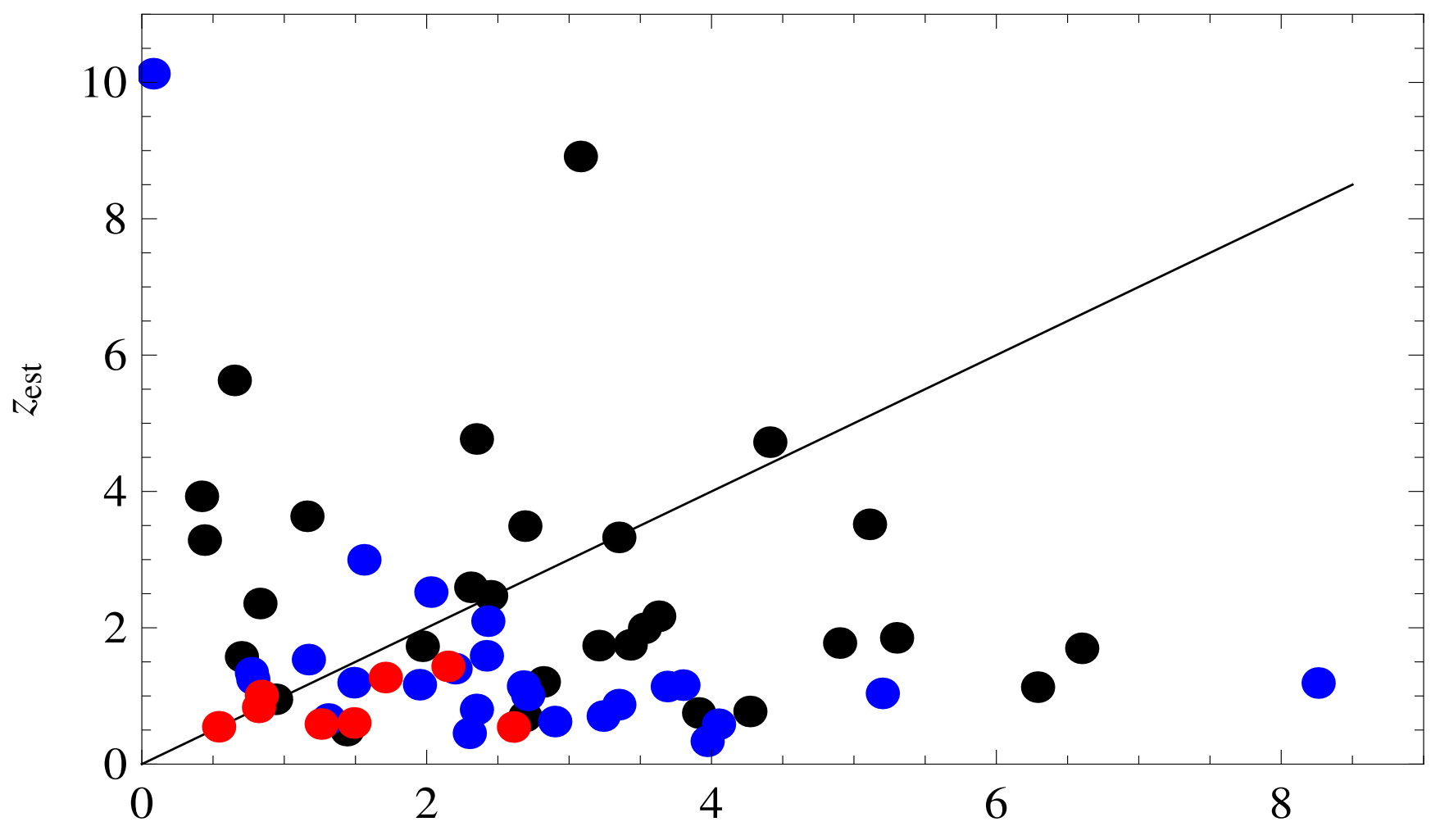

$z_{\text {obs }}$

Figure 38. $z_{o b s}-z_{\text {est }}$ distribution for the 62 LGRBs divided in three $\sigma_{E}$ ranges from Dainotti et al. (2011a): $\sigma_{E} \leq 0.095$ is represented by red points, $0.095 \leq \sigma_{E} \leq 0.3$ is represented by blue points, $0.3 \leq \sigma_{E} \leq 4$ is represented by black points.

Assuming $\Delta z=z_{o b s}-z_{e s t}$, where $z_{o b s}$ and $z_{\text {est }}$ are the observed and the estimated redshifts respectively, it has been shown that $\sim 20 \%$ of GRBs in the E4 sample (black, $0.3 \leq \sigma_{E} \leq 4$, and blue, $0.095 \leq \sigma_{E} \leq 0.3$, points in Fig. 38) has $\left|\Delta z / \sigma\left(z_{\text {est }}\right)\right| \leq 1$. While for the E0095 subsample $28 \%$ has $\left|\Delta z / \sigma\left(z_{\text {est }}\right)\right| \leq 1$, red dots in Fig. 38. The percentage of successful solutions rises to $\sim 53 \%(\sim 57 \%)$ for the E4 (E0095) sample if $\left|\Delta z / \sigma\left(z_{\text {est }}\right)\right| \leq 3$ is considered. The comparison of the results for both the E4 and E0095 samples is proof that $\sigma_{E}$ has no strong influence on the redshift estimate. The reason why the redshift indicator has not yet given successful results depends on the intrinsic scatter of the LT relation. Thus, it is useful to check whether better results can be achieved by increasing the data sample size. For this reason, an E0095 subsample was simulated creating $\left(\log T_{X, a}, \beta_{X, a}, z\right)$ values from a distribution similar to the observed one for the E4 sample. Then, $\log L_{X, a}$ was selected from a Gaussian distribution with mean 
value obtained by the LT relation and with $\sigma_{\text {Gauss }}=\sigma_{\text {int }}$. These values were employed to compute log $F_{X, a}$ and to reproduce the noise for all the quantities so that the relative errors resembled the observations. Then, using Markov chains as input to the redshift estimate formula, it is concluded that only enlarging the sample is not an appropriate methodology to increase the success of the LT relation as a redshift estimator.

In fact, with $\mathcal{N} \simeq 50$, the number of GRBs with $\left|\Delta z / \sigma\left(z_{\text {est }}\right)\right| \leq 1$ first rises to $\sim 34 \%$ and then diminish to $\sim 20 \%$ for $\mathcal{N} \simeq 200$, while $\left\langle\Delta z / z_{o b s}\right\rangle \simeq-17 \%$ for both $\mathcal{N} \simeq 50$ and $\mathcal{N} \simeq 200$. The fact that enlarging the sample does not improve the result could be expected. Indeed, a bigger sample conducts to tighter constraints on the $\left(a, b, \sigma_{i n t}\right)$ values, but does not affect $\sigma_{i n t}$ which is the principal cause of inconsistencies between the observed and the estimated $z$.

Therefore, an alternative way was explored: $\sigma_{\text {int }}$ was decreased and the best fit $(a, b)$ parameters of the E0095 subsample were chosen. In fact, fixing $\sigma_{\text {int }}=0.10$ gives $f\left(\left|\Delta z / z_{\text {obs }}\right| \leq 1\right) \simeq 66 \%$. These outcomes suggested that the LT relation could be employed as a redshift estimator only in the case that a subsample of GRBs could be determined with $\sigma_{\text {int }}=0.10-0.20$. If such a sample is achievable is not clear yet due to the paucity of the E0095 subsample. In fact, it is difficult to find out some useful indicators that can help to define GRBs close to the best fit line of the LT relation. To obtain $\sim 50$ GRBs to calibrate the LT relation with $\sigma_{\text {int }} \sim 0.20$ it has been estimated that a sample of $\sim 600$ GRBs with observed $\left(\log T_{X, a}, \log F_{X, a}, \beta_{X, a}, z\right)$ values is needed. However, even if this is a challenging goal, it may be possible to find out properties of GRB afterglows which enable us to reduce the $\sigma_{\text {int }}$ of the LT relation with a much smaller sample. Finally, an interesting feature would be to correct for the selection effects all the physical quantities of the relations mentioned above. In this manner, it would be possible to average them in order to create a more precise redshift estimator.

\section{COSMOLOGY}

The study of the Hubble Diagram (HD), namely the distribution of the distance modulus $\mu(z)^{8}$ versus $z$ of SNe Ia, opened the way to the investigation of the nature of DE. As it is known from the literature, $\mu(z)$ is proportional to the logarithm of the luminosity distance $D_{L}\left(z, \Omega_{M}, h\right)$ through the following equation:

$$
\mu(z)=25+5 \times \log D_{L}\left(z, \Omega_{M}, h\right) .
$$

In addition, $D_{L}\left(z, \Omega_{M}, h\right)$ is related to different DE EoSs.

\subsection{The problem of the calibration}

One of the most important issues presented in the use of GRB relations for cosmological studies is the so-called circularity problem. Namely, a cosmological model needs to be assumed to compute $D_{L}\left(z, \Omega_{M}, h\right)$. This is due to the fact that local GRBs are not available apart from the case of GRB 980425. Indeed, this kind of GRBs would be observed at $z<0.01$ and their measure would be independent of a particular cosmological setting. This issue could be overcome in three ways: a) through the calibration of these relations by several low $z$ GRBs (in fact, at $z \leq 0.1$ the luminosity distance is not sensitive to the balance of $\Omega_{M}$ and $\Omega_{\Lambda}$ for a given $H_{0}$, where $H_{0}$ is between 65 and 72 ); b) through a solid theoretical model in order to explain the observed $2 \mathrm{D}$ relations. Namely, this would fix their slopes and normalizations independently of cosmology, but this task still has to be achieved; c) through the calibration of the standard candles using GRBs in a narrow redshift range $(\Delta z)$ near a fiducial redshift, $z_{c}$. We here describe some examples on how to overcome the problem of circularity using prompt relations.

The treatment of this problem will be the same once we consider afterglow or prompt-afterglow relations. Liang and Zhang (2006b) suggested a new GRB luminosity indicator, $E_{\gamma, i s o}=a E_{\gamma, p e a k}^{b_{1}} T_{O, a}^{b_{2}}$, different from the previous GRB luminosity indicators that are generally written in the form of $L=a \prod x_{i}^{b_{i}}$, where a is the normalization, $x_{i}$ is the i-th observable, and $b_{i}$ is its corresponding power law index. It was demonstrated that while $a$ relies on the cosmological parameters, this is not the case for $b_{1}$ and $b_{2}$ until $\Delta z$ is sufficiently little, see Fig. 39. The choice of $\Delta z$ for a given GRB sample could be evaluated depending on its dimension and the errors on the variables. The most suitable approach would be to assemble GRBs within a small redshift range around a central $z_{c}\left(z_{c} \sim 1\right.$ or $z_{c} \sim 2$ ), because the GRB $z$ distribution peaks in this interval (see also Wang et al. 2011 and Wang et al. 2015).

In addition, also Ghirlanda et al. (2006) defined the luminosity indicator $E_{\gamma, \text { peak }}=a \times E_{\gamma, \text { cor }}^{b}$ using the log $E_{\gamma, p e a k}-$ $\log E_{\gamma, \text { cor }}$ relation (Ghirlanda et al. 2004), where

$$
E_{\gamma, c o r}=\left(1-\cos \theta_{j e t}\right) \times 4 \pi \times D_{L}^{2}\left(z, \Omega_{M}, h\right) \times S_{\gamma, p r o m p t} /(1+z)^{2}
$$

\footnotetext{
8 The difference between the apparent magnitude $\mathrm{m}$, ideally corrected from the effects of interstellar absorption, and the absolute magnitude $\mathrm{M}$ of an astronomical object.
} 

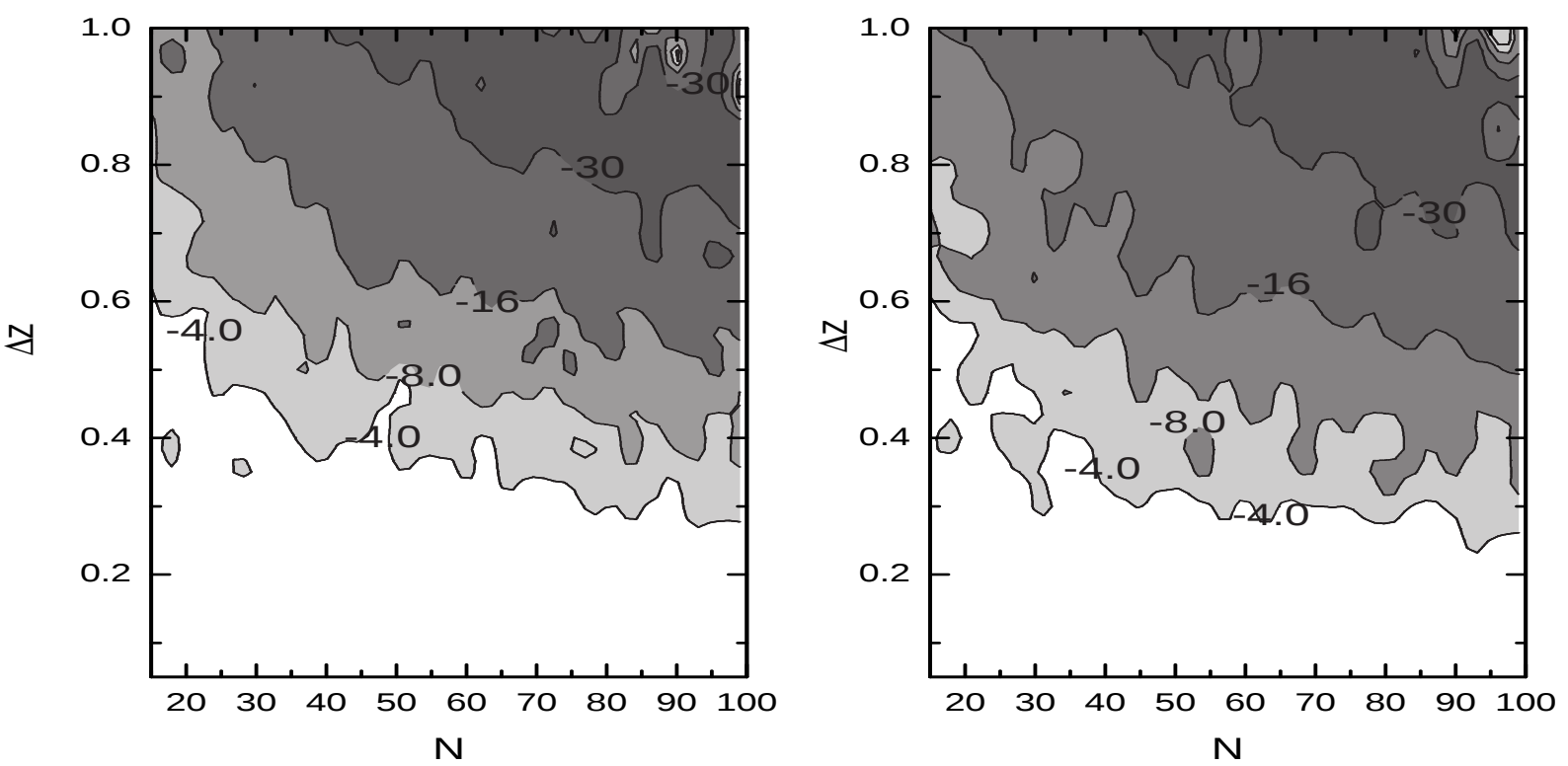

Figure 39. "Distribution of $\log P$ in the (N, $\Delta z)$ plane from Liang and Zhang (2006b). The grey contours mark the areas where the dependencies of $b_{1}$ and $b_{2}$ on $\Omega_{M}$ are statistically significant $\left(\mathrm{P}<10^{-4}\right)$. The white region is suitable for the calibration purpose".

is the energy corrected for the beaming factor and $\theta_{\text {jet }}$ is the opening angle of the jet. They calculated the minimum number of GRBs $(\mathrm{N})$, within $\Delta z$ around a certain $z_{c}$, needed to calibrate the relation, considering a sample of 19 GRBs detected mostly by Beppo-SAX and Swift. Particularly, they fitted the relation for each value of $\Omega_{M}$ and $\Omega_{\Lambda}$ using a set of $\mathrm{N}$ GRBs distributed in the interval $\Delta z$ (centered around $z_{c}$ ). If the variation of the slope, $\mathrm{b}$, is less than $1 \%$ the relation is assumed calibrated. $N, \Delta z$ and $z_{c}$ are free parameters. They checked several $z_{c}$ and distinct $z$ dispersions $\Delta z \in(0.05,0.5)$ by Monte Carlo simulations. At every $z$ the smaller the $\mathrm{N}$ the bigger the variation of the slope, $\Delta b$ (for the same $\Delta z$ ), because the relation is more scattered. On the other hand, for greater $z_{c}$ a tinier $\Delta z$ is necessary to maintain $\Delta b$ in its little state. Finally, they found that 12 GRBs with $z \in(0.9,1.1)$ can be sufficient to calibrate the slope of the $\log E_{\gamma, \text { peak }}-\log E_{\gamma, \text { cor }}$ relation. Instead, at $z_{c}=2$ a narrower redshift bin is needed, for example $z \in(1.95,2.05)$.

However, this method might becomes unsuccessful, because the sample size of the observed GRBs is not sufficiently big. Another method for a model-independent calibration may be obtained employing SNe Ia as distance indicators. This method is based on the assumption that a GRB at redshift $\mathrm{z}$ must have the same distance modulus $\mu(z)$ of a SNe Ia at the same redshift. In this way, GRBs should be considered as complementary to SNe Ia at very high z, thus allowing for the construction of a very long distance ladder. Therefore, interpolating the SNe Ia HD provides the value of $\mu(z)$ for a subsample of GRBs with $z \leq 1.4$, which can be employed for the calibration of the 2D relations (Kodama et al. 2008; Liang et al. 2008; Wei and Zhang 2009). This value is given by the formula:

$$
\begin{aligned}
\mu(z) & =25+(5 / 2)(\log y-k) \\
& =25+(5 / 2)(a+b \log x-k),
\end{aligned}
$$

where $y=k D_{L}^{2}\left(z, \Omega_{M}, h\right)$ is a given quantity with $k$ a redshift independent constant, and $a$ and $b$ are the relation parameters. Presuming that this calibration is redshift independent, the HD at higher $z$ can be constructed using the calibrated relations for the other GRBs in the data set.

Finally, Li and Hjorth (2014) analyzed the light curves of 8 LGRBs associated with SNe finding a relation between the peak magnitude and the decline rate at 5, 10 and 15 days as in SNe Ia. However, from the comparison with the well-known relation for SNe Ia (Phillips 1993), it was pointed out that these two objects have two different progenitors. More importantly, this discovery allowed to use GRBs associated with SNe as possible standard candles. In addition, Cano (2014) investigated the optical light curves of 8 LGRBs associated with SNe discovering evidence of a relation between their luminosity and the width of the GRB light curves relative to the template of the well-known SN 1998bw. This result also confirmed the possibility of using GRBs associated with SNe as standard candles. 


\subsection{Applications of GRB afterglow relations}

In this section, we describe some applications to cosmology only for the LT relation, because this is the only afterglow relation that has been used so far as a cosmological probe. However, the method is very general and it can be employed for all the other relations presented in the review. The idea to use afterglow GRBs phase as cosmological rulers was proposed for the first time in 2009, when the LT relation was used to derive a new HD (Cardone et al. 2009, 2010). More specifically, Cardone et al. (2009) revised the data set used in Schaefer (2007) appending the LT relation. They used a Bayesian fitting method, similar to that used in Firmani et al. (2006) for the $\log E_{\gamma, \text { peak }}-\log E_{\gamma, c o r}$ relation, to calibrate the different GRB relations known at that time assuming a fiducial $\Lambda \mathrm{CDM}$ model compatible with the data provided by the Wilkinson Microwave Anisotropy Probe, WMAP5.

A new HD including 83 objects was obtained (69 from Schaefer (2007) plus 14 new GRBs obtained by the LT relation) computing the mean performed over six relations $\left(\log E_{\gamma, \text { cor }}-\log E_{\gamma, \text { peak }}, \log L_{\gamma, \text { iso }}-\log V\right.$, with $V$ the variability which measures the difference between the observed light curve and a smoothed version of that light curve, $\log L_{X, a}-\log T_{X, a}^{*}$, $\log L_{\gamma, \text { iso }}-\log \tau_{\text {lag }}$, with $\tau_{\text {lag }}$ the difference in arrival time to the observer of the high energy photons and low energy photons, $\log L_{\gamma, i s o}-\log \tau_{R T}$, with $\tau_{R T}$ the shortest time over which the light curve increases by the $50 \%$ of the peak flux of the pulse, and $\left.\log L_{\gamma, \text { iso }}-\log E_{\gamma, \text { peak }}\right)$.

To elude the circularity problem, local regression was run to calculate $\mu(z)$ from the newest SNe Ia sample containing $307 \mathrm{SNe}$ Ia in the range $0.015 \leq z \leq 1.55$. Indeed, the GRB relations mentioned before were calibrated while considering only GRBs with $z \leq 1.4$ in order to cover the same redshift range spanned by the SNe Ia data. This $\mathrm{SNe}$ Ia sample is the input for the local regression estimate of $\mu(z)$.

The basic idea of the local regression analysis consists of several stages described in Cardone et al. (2009). To find out which are the optimal parameters of this procedure, a large sample of simulations was carried out. They set the value of the model parameters $\left(\Omega_{M}, w_{0}, w_{a}, h\right)$, with $w_{0}$ and $w_{a}$ given by the coefficient of the DE EoS $w(z)=w_{0}+w_{a} z(1+z)^{-1}$ (Schaefer 2007), in the ranges $0.15 \leq \Omega_{M} \leq 0.45,-1.5 \leq w_{0} \leq-0.5,-2.0 \leq w_{a} \leq 2.0$ and $0.60 \leq h \leq 0.80$. For each $z$ value, $\mu\left(z_{i}\right)$ was selected from a Gaussian distribution centered on the predicted value and with $\sigma_{\text {int }}=0.15$, consistent with the $\sigma_{\text {int }}$ of the SNe Ia absolute magnitude. This way, a mock catalogue with the same $z$ and error distribution of the SNe sample was built. Each $\mu(z)$ value derived from this procedure is compared to the input one. The local regression method correctly produces the underlying $\mu(z)$ at each $z$ from the SNe Ia sample, whichever is the cosmological model.

Furthermore, comparing their HD to the one derived by Schaefer (2007), referred as the Schaefer HD, they have updated the Schaefer HD in three ways, namely updating the $\Lambda$ CDM model parameters, using a Bayesian fitting procedure and adding the LT relation. To analyze the influence of these changes, the sample of 69 GRBs adopted by Schaefer (2007) was also used and the distance moduli were computed with the new calibration, but without considering the LT relation. It was found that $\mu_{\text {new }} / \mu_{\text {old }}$ is close to 1 within $5 \%$. Thus, this calibration procedure has not modified the results.

In conclusion, it was pointed out that the $\mu(z)$ for each of the GRBs in common to Schaefer (2007) and Dainotti et al. (2008) samples is compatible with the one computed using the set of Schaefer (2007) relations. Therefore, no systematic bias is added by also considering the LT relation. On the other hand, the addition of the LT relation to the pre-existing ones not only decreases the errors on $\mu(z)$ by $\sim 14 \%$, but also expands the data set from 69 to 83 GRBs. While Cardone et al. (2009) added the LT relation to a set of other 5 known prompt emission relations, Cardone et al. (2010) used instead the LT relation alone (66 LGRBs) or in combination with other cosmological tools in order to find some constraints on the cosmological parameters at large $z$. The GRBs were divided in E0095 and E4 samples, indicating that the introduction of the LT relation alone also provides constraints compatible with previous outcomes, since the HD spans over a large redshift range $(0.033,8.2)$.

Furthermore, considering three different cosmological models, namely the $\Lambda \mathrm{CDM}$, the CPL (Chevallier and Polarski 2001) and the quintessence (QCDM), it was discovered that the $\Lambda$ CDM model is preferred. To better show the impact of GRBs, the fit was repeated only with other probes, such as SNe Ia or Baryon acoustic Oscillations, excluding the

GRBs. The addition of GRBs does not significantly narrow the parameters confidence ranges, but GRBs drive the constraints on $w_{a}$ to 0 . This result indicates that the consideration of a big sample of E0095 GRBs may lead to a constant EoS DE model.

In addition, we may note that, different from what was done in the literature at the time of their publication, the HD for the E0095 and E4 samples is the only GRB HD built with a single relation in the afterglow containing a statistically significant sample.

Furthermore, the LT relation does not request the mix of several relations to rise the number of GRBs with a known 
$\mu(z)$. In fact, each relation is influenced by its own biases and intrinsic scatter; therefore, using all of them in the same HD can affect the evaluation of the cosmological parameters. The $\sigma_{\text {int }}$ of the LT relation may be considerably decreased if only the E0095 subsample is analyzed. However, considering the whole sample of 66 LGRBs, Cardone et al. (2010) constrained $\Omega_{M}$ and $H_{0}$ obtaining values compatible with the ones presented in the literature.

This analysis clearly claimed that the LT relation can be considered for building a GRB HD without adding any bias in the study of the cosmological parameters. Equivalent findings were achieved considering E0095 GRBs even if they are just $12 \%$ of the whole sample. Therefore, a further investigation of E0095 GRBs can boost their use as standard sample for studying the DE mystery.

As a further development, Dainotti et al. (2013b) pointed out to what extent a separation of $5 \sigma$ above and below the intrinsic value, $b_{i n t}=-1.07_{-0.14}^{+0.09}$, of the slope of the LT relation can influence the cosmological results.

For this study, a simulated data set of $101 \mathrm{GRBs}$ obtained through a Monte Carlo simulation was collected assuming $b=-1.52, \sigma_{\text {int }}=0.93$ (larger than the scatter computed from the original data set, namely $\sigma_{\text {int }}=0.66$ ), and the fiducial $\Lambda$ CDM flat cosmological model with $\Omega_{M}=0.291$ and $H_{0}=71 \mathrm{Km} \mathrm{s}^{-1} \mathrm{Mpc}^{-1}$. They investigated how much the scatter in the cosmological parameters can be diminished if, instead of the total sample (hereafter Full), a highly luminous subsample (hereafter High Luminosity) is considered, constrained by the condition that $\log L_{X, a} \geq 48.7$. The choice of this selection cut at a given luminosity is explained in Dainotti et al. (2013a), who showed that the local luminosity function is similar to the observed luminosity one for $\log L_{X, a} \geq 48$.

The methodology is similar to what has been done by Amati et al. (2008) for the $\log E_{\gamma, p e a k}-\log E_{\gamma, i s o}$ relation, namely the fit has been performed varying simultaneously both the calibration parameters, $p_{G R B}=\left(a, b, \sigma_{i n t}\right)$, and the cosmological parameters, $p_{c}=\left(\Omega_{M}, \Omega_{\Lambda}, w_{0}, w_{a}, h\right)$, each time for a given model in order to correctly take this issue into account.

In order to have stronger limits on the cosmological parameters two samples were added to the data set, the $H(z)$ sample $\left(H(z)=H_{0} \times \sqrt{\Omega_{M}(1+z)^{3}+\Omega_{k}(1+z)^{2}+\Omega_{\Lambda}}\right)$ over the redshift range $0.10 \leq z \leq 1.75$ (Stern et al. 2010 ) and the Union 2.1 SNe Ia sample containing 580 objects over the redshift range $0.015 \leq z \leq 1.414$ (Suzuki et al. 2012 ). A Markov Chain Monte Carlo (MCMC) method was used, running three parallel chains and applying the GelmanRubin test ${ }^{9}$ in order to analyze the convergence for an assumed cosmological model characterized by a given set of cosmological parameters $p_{c}$ to be determined.

From this statistical analysis results regarding the Full GRB sample, $b, a$ and $\sigma_{i n t}$ of the LT relation are independent of the chosen cosmological model and the presence of the SNe Ia and $H(z)$ data in the sample. In addition, even if a $5 \sigma$ scatter in $b_{i n t}$ is assumed, the results for the Full sample are in agreement with earlier outcomes (Dainotti et al. 2008, 2011a) where exclusively flat models were assumed.

On the other hand, due to the wide errors on the simulated data, the cosmological parameters are not emerging in the calibration procedure. However, the signature of the cosmology will appear considering a greater data set with low errors on $\left(\log T_{X, a}^{*}, \log L_{X, a}\right)$.

Furthermore, for the Full sample, it was studied how much the deviation from the $b_{\text {int }}$ of the LT relation influences the cosmological parameters. To analyze this problem, a model parameterized in terms of the present day values of $\Omega_{M}, \Omega_{\Lambda}$ and $H_{0}$ was considered.

Although $h$ is comparable with the values from both the local distance estimators (Riess et al. 2009) and CMBR data (Komatsu et al. 2011), the median values for $\left(\Omega_{M}, \Omega_{\Lambda}\right)$ are broader if compared to a fiducial $\Omega_{M} \sim 0.27$ recovered in earlier works (Davis et al. 2007). For this reason, considering for the Full sample, a distinct $b_{i n t}$ will lead to a disagreement of $13 \%$ with the best value of the $\Omega_{M}$ parameter (see the upper panels of Fig. 40). Even if the median values of the fit for the sample that also has SNe Ia and $H(z)$ data do not conduct towards flat models, a spatially flat Universe accords with, for example, the WMAP7 cosmological parameters within $95 \%$ giving $\Omega_{k}=-0.080_{-0.093}^{+0.071}$. This difference can be deduced, because in this case it is not possible to distinguish among flat and not flat models and this distinction is still not possible when SNe Ia data are present in the fit. Thus, constraining the model to be spatially flat, but shaping the DE EoS with $w(z)$, leads to a couple $\left(w_{0}, w_{a}\right)$ completely different irrespective of whether SNe Ia and $H(z)$ data are considered or not in the sample. Regarding instead the High Luminosity subsample, the limits on the calibration parameters mostly do not depend on either the used cosmological model or if $\mathrm{SNe}$ Ia and $H(z)$ data are considered in the sample. Furthermore, for the High Luminosity subsample it is shown that adding the SNe Ia and $H(z)$ data does not ameliorate the constraints on the calibration parameters.

Finally, the Full sample outcomes are comparable to those of the flat cosmological model for the SNe Ia sample, while

${ }^{9}$ The Gelman-Rubin diagnostics relies on parallel chains to test whether they all converge to the same posterior distribution. 


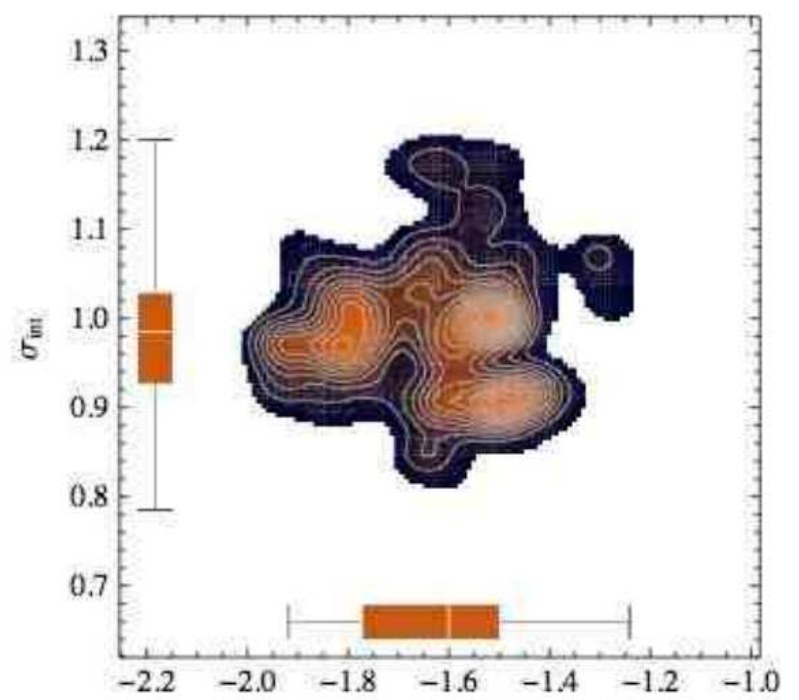

a

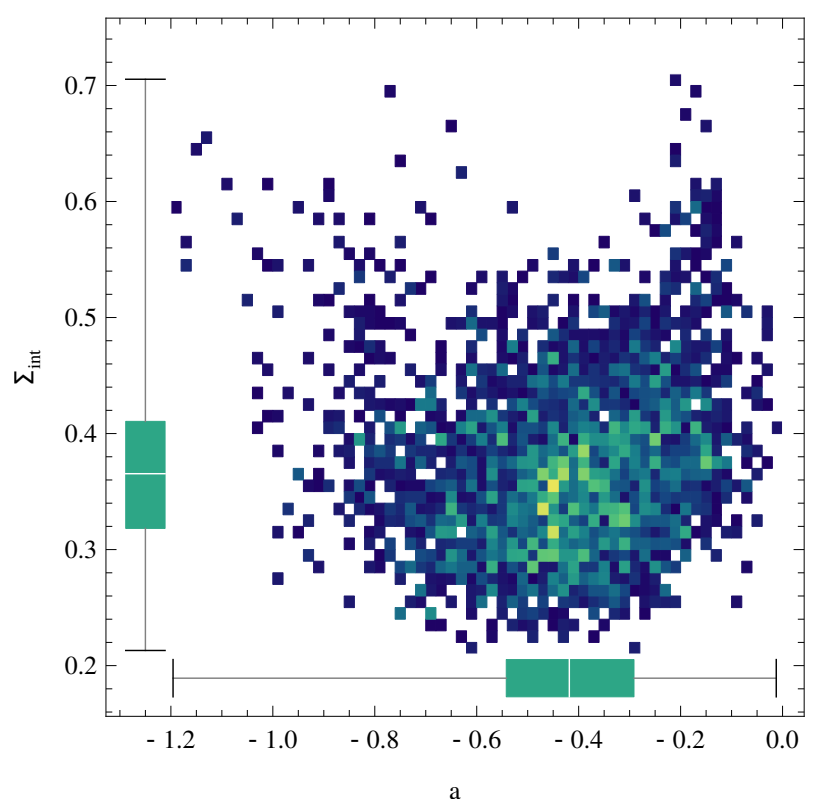

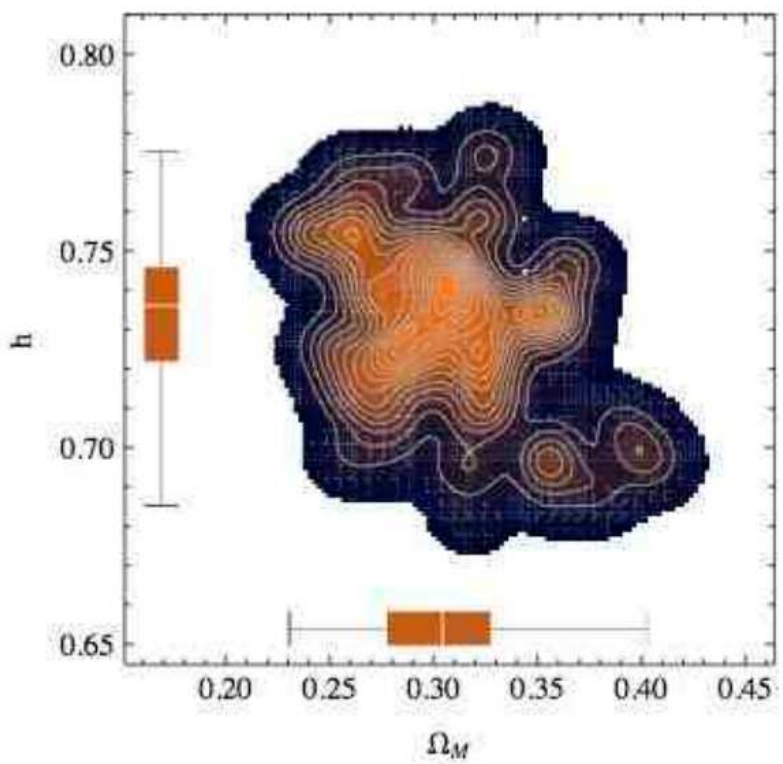

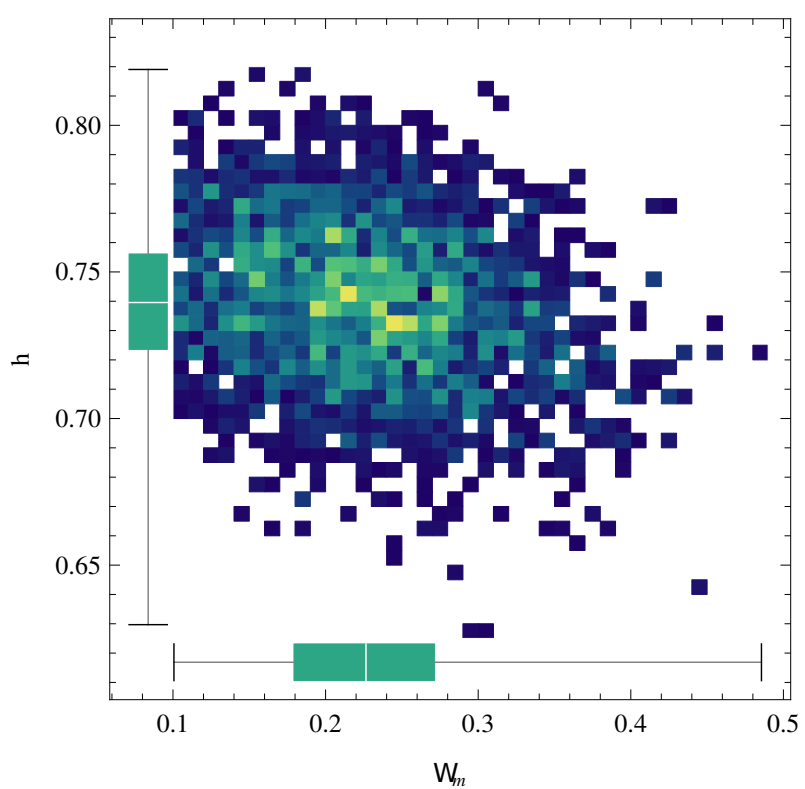

Figure 40. Upper left panel: "regions of confidence for the marginalized likelihood function $\mathcal{L}(b, \sigma)$ from $\mathrm{Dainotti}$ et al. (2013b), obtained marginalizing over $a$ and the cosmological parameters using the Full sample. The bright brown regions indicate the 1 (full zone) and $2 \sigma$ (bright grey) regions of confidence respectively. On the axes are plotted the box-and-whisker diagrams relatively to the $b$ and $\sigma_{i n t}$ parameters: the bottom and top of the diagrams are the 25th and 75th percentile (the lower and upper quartiles, respectively), and the band near the middle of the box is the 50th percentile (the median)". Upper right panel: "regions of confidence for the marginalized likelihood function $\mathcal{L}\left(\Omega_{M}, h\right)$, obtained using the Full sample, from Dainotti et al. (2013b)". Bottom left panel: "regions of confidence for the marginalized likelihood function $\mathcal{L}(b, \sigma)$ from Dainotti et al. (2013b), obtained marginalizing over $a$ and the cosmological parameters for the High Luminosity sample. The bright brown regions indicate the $1 \sigma$ (full zone) and $2 \sigma$ (bright grey) regions of confidence respectively. On the axes are plotted the box-and-whisker diagrams relatively to the $b$ and $\sigma_{\text {int }}$ parameters: the bottom and top of the diagrams are the 25th and 75 th percentile (the lower and upper quartiles, respectively), and the band near the middle of the box is the 50th percentile (the median)". Bottom right panel: "regions of confidence for the marginalized likelihood function $\mathcal{L}\left(\Omega_{M}, h\right)$, obtained using the High Luminosity sample, from Dainotti et al. (2013b)".

the High Luminosity subsample diverges by $5 \%$ in the value of $H_{0}$ as computed in Petersen et al. (2010), and the scatter in $\Omega_{M}$ is underestimated by $13 \%$, see the bottom panels of Fig. 40. In conclusion, an optimal procedure is to consider a High luminosity subsample provided by a cut exactly at $\log L_{X, a}=48$; otherwise, the luminosity and time evolutions should be added in the computation of the cosmological parameters.

Later, another application of GRBs to cosmology is presented in Postnikov et al. (2014) where the DE EoS was 
analyzed as a function of $z$ without assuming any a priori $w(z)$ functional form.

To build a GRB $(\mu, z)$ diagram, 580 SNe Ia from the Union 2.1 compendium (Suzuki et al. 2012) were used together with 54 LGRBs in the overlapping redshift $(z \leq 1.4$ see the left panel of Fig. 41) region between GRBs and SNe Ia. In addition, a standard $w=-1$ cosmological model was assumed.
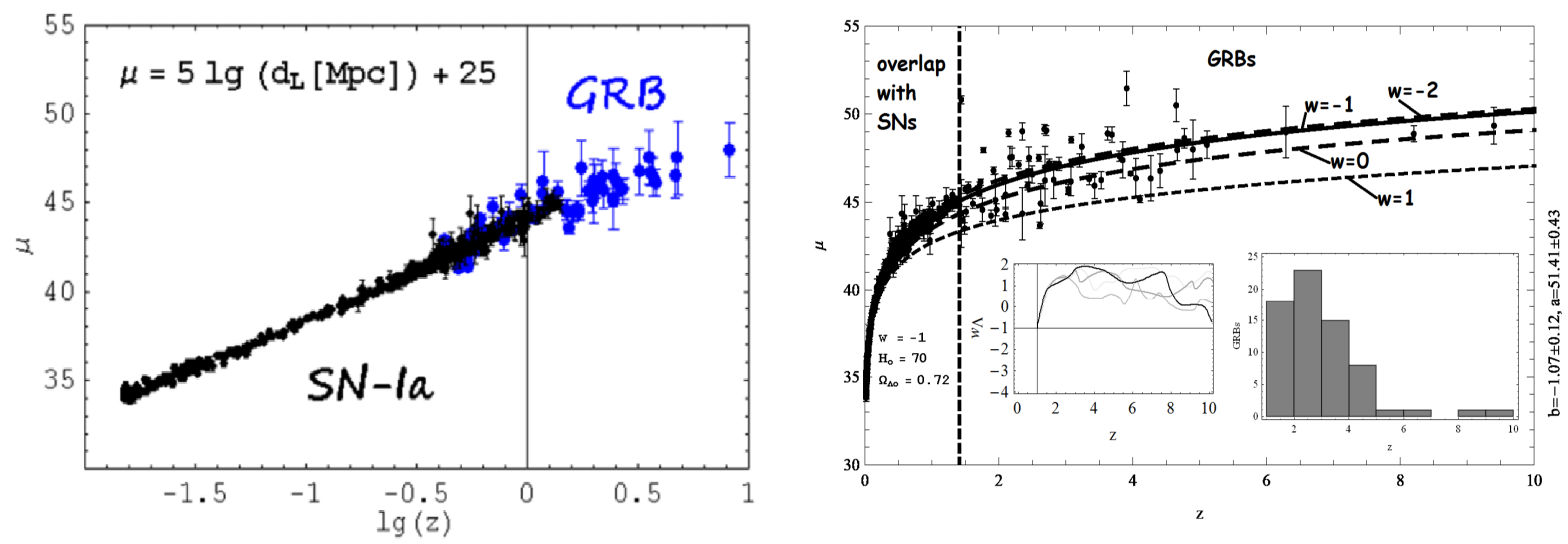

Figure 41. Left panel: " $\left(z_{j}, \mu_{j} \pm \Delta \mu_{j}\right)$ for SNe Ia from Postnikov et al. (2014). GRBs are inferred from the relation assuming a flat $w=-1$ cosmology and stand out only from their larger error bars, no discontinuity is evident, implying a first order consistency of a $w=-1$ model out to very high $z$. The SNe Ia data were taken from the Union 2.1 compendium (Suzuki et al. 2012)". Right panel: "distance ladder from Postnikov et al. (2014). GRBs in the SNe Ia overlap redshift range, where cosmology is well constrained, are used to calculate the GRB intrinsic correlation coefficient. This relation is then used to calculate $D_{L}\left(z, \Omega_{M}, h\right)$ for high $z$ GRBs from their X-ray afterglow luminosity curves. Standard constant $w$ solutions are shown for reference. Vertical dashed line marks farthest SNe Ia event. Inset to the right shows a histogram of the GRB sample distribution in $z$. Inset to the left shows resulting most probable EoS, together with a small sample of models probed, confidence intervals are so large, that only extreme variations with respect to $w=-1$ can be excluded".

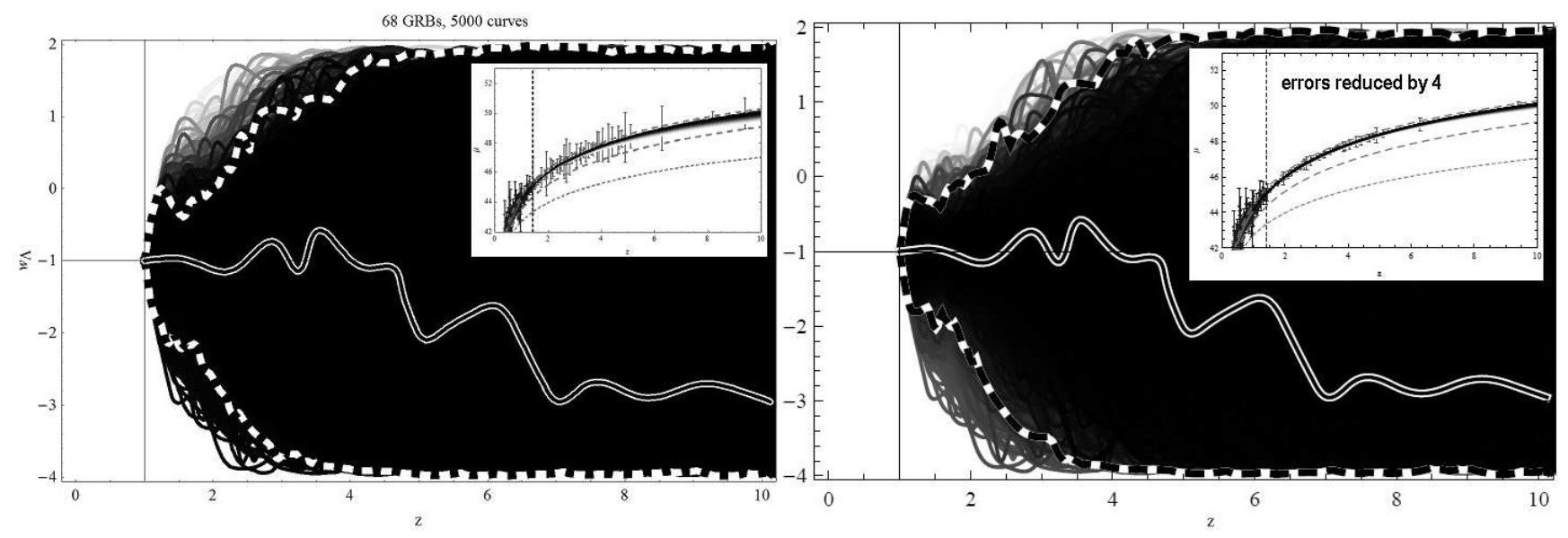

Figure 42. "Tree of $w(z>1)$ curves inferred from synthetic GRB samples constructed for $w(z)=-1$ cosmologies in Postnikov et al. (2014), showing to what extent correlated GRB errors constrain EoS at high $z(z>1)$. In the left side plot GRB errors taken from actual data are used, while in the right side plot GRB errors reduced by a factor of 4 are considered".

One order of magnitude expansion in redshift interval is supplied by the GRB data set considering the correlation coefficients obtained for the SNe Ia. This detail allows for the enlargement of the cosmological model out to $z=8.2$. In fact, a relation was found given by:

$$
\log L_{X, a}=53.27_{-0.48}^{+0.54}-1.51_{-0.27}^{+0.26} \times \log T_{X, a}^{*}
$$

with $\rho=-0.74$ and $P=10^{-18}$.

Postnikov et al. (2014) used a Bayesian statistical analysis, similarly to Firmani et al. (2006) and Cardone et al. (2010), in which the hypothesis is related to a particular $w(z)$ function with the selection of $H_{0}$ and the present DE density parameter, $\Omega_{\Lambda 0}$. The assumption of isotropy for the cosmological model, reliable limits on the EoS and also a fixed 
value for $w(z)$ in the $z \leq 0.01$ redshift interval were employed. In addition, a huge number of randomly chosen $w(z)$ models were used.

To test the procedure, their pattern is verified through the simulated data sets obtained from several input cosmological models with relative errors and $z$ distribution equal to the real data. Through this procedure, employing the LT relation, a data set of GRBs detected by the Swift satellite, with $z$ from 0.033 to 9.44, was adopted (see inset in the right panel of Fig. 41). Thus, it is possible to investigate the history of the Universe out to $z \approx 10$. (However, an additional analysis would be beneficial if we would consider the sample without the GRB at $z=9.4$. We note that indeed in Cardone et al. (2010) a sample of canonical GRBs was used in which this burst has not been included).

In order to do that, they simulated 2000 constant EoSs uniformly spaced between $-4 \leq w_{\Lambda} \leq 2$, with $w_{\Lambda}$ the DE EoS. Beginning from SNe Ia data sample, a precise solution was found to be in agreement with the cosmological constant and a small confidence interval, $w=-0.99 \pm 0.2$, see the right panel of Fig. 41. Furthermore, it is shown that assuming also that the BAO limits do not differ from the solution of the EoS, but it considerably decreases the confidence interval $(w=-0.99 \pm 0.06)$. In fact, the insertion of the BAO notably constrained the confidence region of the solutions, especially for the present DE density parameter, giving $\Omega_{\Lambda 0}=0.723 \pm 0.025$.

As a further step, the $w(z)$ model which leads to the best evaluation of $D_{L}\left(z, \Omega_{M}, h\right), z$ of the SNe Ia sample and the $\mathrm{BAO}$ constraints needs to be selected. The confidence region of the allowed $w(z)$ curves is significantly constrained taking into account also the BAO data.

Afterwards, also considering that GRB data should constrain the cosmological parameters, apart from obtaining one order of magnitude expansion in the redshift range, it was extremely difficult to constrain the high $z w(z)$ functional form, considered the paucity of points over a broad redshift interval and the error bars related to these data. This is visible in the left panel of Fig. 42, where a simulated GRB data set having the same $z$ distribution and error bars as the real data, but with assumed $w=-1$ Universe, is provided. It is noted that only strong $w(z)$ fluctuations are not allowed. Then, decreasing the errors by a factor of 4 led to more intriguing high $z$ DE constraints, see the right panel of Fig. 42.

In addition, the small number of elements in the SNe Ia overlapping region indicated broad error bars on the GRB correlation coefficients. Meanwhile, the broad error bars for high $z$ GRBs generated a very flat probability distribution (represented by the uniform black shading area in the left panel of Fig. 42) for the several EoSs checked. Therefore, there will be great interest for the $1<z<4$ region of the GRB HD as soon as the GRB data set is enlarged and the quality of data is upgraded.

\section{SUMMARY AND DISCUSSION}

From the analysis of the relations mentioned in previous sections, it is visible that:

1. The accretion model (Cannizzo and Gehrels 2009; Cannizzo et al. 2011) and the magnetar model (Usov 1992; Dall'Osso et al. 2011; Rowlinson and O'Brien 2012) seem to give the best explanation of the Dainotti relation (giving best fit slopes $-3 / 2$ and -1 respectively). The magnetar model seems to be favoured compared to the accretion one, because the intrinsic slope computed in Dainotti et al. (2013a) is exactly $-1.07_{-0.9}^{+0.14}$.

2. A more complex jet structure is needed for interpreting the $\log L_{O, 200 s}-\alpha_{O,>200 s}$ relation (Oates et al. 2012). Indeed, Oates et al. (2012) showed that the standard afterglow model cannot explain this relation, especially taking into account the closure relations (Sari et al. 1998), which relate temporal decay and spectral indices. Therefore, in order to interpret their results, they claimed either the presence of some features of the central engine which dominate the energy release or that the observations were made by observers at different angular distances from the source's axis. Dainotti et al. (2013a) pointed out a similarity between the $\log L_{O, 200 \mathrm{~s}}-\alpha_{O,>200 \mathrm{~s}}$ relation and the $L_{X}-T_{a}^{*}$ relation, making worthy of investigating the possibility of a single physical mechanism inducing both of them.

3. In the external shock model the $L_{X}\left(T_{a}\right)-L_{\gamma, \text { iso }}$ and the $L_{X, \text { peak }}-L_{X}\left(T_{a}\right)$ relations cannot lead to a net distinction among constant or wind type density media, but they are able to exclude so far the thin shell models and to favour the thick shell ones. Among the models that very well describe the $L_{X}\left(T_{a}\right)-L_{\gamma, i s o}$ and the $L_{X, \text { peak }}-L_{X}\left(T_{a}\right)$ relations there is the one by Hascoët et al. (2014). They investigated the standard FS model with a wind external medium and a microphysics parameter $\epsilon_{e} \propto n^{-\nu}$, and they found out that for values $\nu \approx 1$ is possible to reproduce a flat plateau phase, and consequently the relations mentioned above. This shows how important the study of correlations especially with the aim of discriminating among models. 
4. In regard to the prompt-afterglow relations, mentioned in section 4, involving the energies and the luminosities for the prompt and the afterglow phases, it is pointed out that they help to interpret the connection between these two GRB phases. For example, Racusin et al. (2011) pointed out that the fraction of kinetic energy transferred from the prompt phase to the afterglow one, for BAT-detected GRBs, is around 10\%, in agreement with the analysis by Zhang et al. (2007a). However, from the investigation of these relations, the synchrotron radiation process seems to not explain completely the observations, and also the scatter present in these relations is significant. Therefore, further analysis will be useful.

5. The study of the $L_{O, \text { peak }}^{F}-T_{O, \text { peak }}^{* F}$ relation sheds light on the nature of the flares in the GRB light curves. From the analysis carried on by Li et al. (2012), it was found out that the flares are additional and distinct components of the afterglow phase. They also claimed that a periodically-emitting energy central engine can explain the optical and $\gamma$-ray flares in the afterglow phase.

6. One of the greatest issues that may undermine the GRB relations as model discriminators and as cosmological tools are selection bias and the evolution with the redshift of the physical quantities involved in these relations. An example of selection biases is given by Dainotti et al. (2013a), who used the Efron and Petrosian (1992) method to deal with the redshift evolution of the X-ray luminosity and the time, to evaluate the intrinsic $L_{X}-T_{a}^{*}$ relation. Furthermore, Dainotti et al. (2015a) assumed an unknown efficiency function for the detector and investigated the biases due to the detector's threshold and how they affect the X-ray luminosity and the time measurements. The methods described can be also useful to deal with the selection effects for the optical luminosity and in the $\log L_{O, 200 \mathrm{~s}}-\alpha_{O,>200 \mathrm{~s}}$ relation and any other relation.

7. Regarding the use of correlations as cosmological tools, we still have to further reduce the scatter of the GRB measurements and the dispersion of the relations themselves to allow GRBs to be complementary with the measurement of SNe Ia. Indeed, the redshift evolution effect and the threshold of the detector can generate relevant selection biases on the physical quantities which however we know how to treat analytically with robust statistical techniques as we have shown in several sections. Nevertheless, more precise calibration methods, with the help of other cosmological objects, and more space missions dedicated to detect faint GRBs and GRBs at high redshift (for example the future SVOM mission) can shed new light on the use of GRBs as cosmological tools. Lastly, other open questions are concerned with how much cosmological parameters can reduce their degeneracy adding GRBs into the set of cosmological standard candles. For example, different results of the value of $w$ can lead to scenarios which can be compatible with a non-flat cosmological model.

\section{CONCLUSIONS}

In this work, we have summarized the bivariate relations among the GRB afterglow parameters and their characteristics in order to discuss their intrinsic nature and the possibility to use them as standardizable candles. It has been shown with different methodologies that some of the relations presented are intrinsic. However, the intrinsic slope has been determined only for a few relations. For the other relations, we are not aware of their intrinsic slopes and consequently how far the use of the observed relations can influence the evaluation of the theoretical models and the "best" cosmological settings (Dainotti et al. 2013b). Therefore, the estimate of the intrinsic relations is crucial for the determination of the most plausible model that can explain the plateau phase and the afterglow emission.

In fact, though there are several theoretical interpretations describing each relation, as we have shown, in many cases, more than one is viable. This result indicates that the emission processes that rule the GRBs still have to be further investigated. To this end, it is necessary to use the intrinsic relations and not the observed ones affected by selection biases to test the theoretical models. Moreover, the pure afterglow relations have the advantage of not presenting the double truncation in the flux limit, thus facilitating the correction for selection effects and their use as redshift estimators and cosmological tools.

A very challenging future step would be to use the corrected relations as a reliable redshift estimator and to determine a further estimate of $H_{0}, \Omega_{\Lambda}$ and $w$. In particular, it is advisable to use all the afterglow relations which are not yet employed for cosmological studies as new probes, after they are corrected for selection biases, in order to reduce the intrinsic scatter as it has been done in Schaefer (2007) for the prompt relations.

\section{ACKNOWLEDGMENTS}

This work made use of data supplied by the UK Swift Science Data Centre at the University of Leicester. We thank S. Capozziello for fruitful comments. M.G.D is grateful to the Marie Curie Program, because the research 
leading to these results has received funding from the European Union Seventh FrameWork Program (FP7-2007/2013) under grant agreement N 626267. R.D.V. is grateful to the Polish National Science Centre through the grant DEC2012/04/A/ST9/00083.

\section{REFERENCES}

L. Amati and M. Della Valle. Measuring Cosmological

Parameters with Gamma Ray Bursts. International Journal of Modern Physics D, 22:1330028, December 2013. doi:10.1142/S0218271813300280

L. Amati, F. Frontera, M. Tavani, J. J. M. in't Zand, A. Antonelli, E. Costa, M. Feroci, C. Guidorzi, J. Heise, N. Masetti, E. Montanari, L. Nicastro, E. Palazzi, E. Pian, L. Piro, and P. Soffitta. Intrinsic spectra and energetics of BeppoSAX Gamma-Ray Bursts with known redshifts. $A \mathscr{E} A$, 390:81-89, July 2002. doi:10.1051/0004-6361:20020722

L. Amati, M. Della Valle, F. Frontera, D. Malesani, C. Guidorzi, E. Montanari, and E. Pian. On the consistency of peculiar GRBs 060218 and 060614 with the E_p,i - E_iso correlation. A\&A, 463:913-919, March 2007. doi:10.1051/0004-6361:20065994.

L. Amati, C. Guidorzi, F. Frontera, M. Della Valle, F. Finelli, R. Landi, and E. Montanari. Measuring the cosmological parameters with the $\mathrm{E}_{p, i}-\mathrm{E}_{\text {iso }}$ correlation of gamma-ray bursts. MNRAS, 391:577-584, December 2008. doi:10.1111/j.1365-2966.2008.13943.x

J.-L. Atteia. A simple empirical redshift indicator for gamma-ray bursts. $A \& A, 407:$ L1-L4, August 2003. doi:10.1051/0004-6361:20030958.

E. Berger. The Prompt Gamma-Ray and Afterglow Energies of Short-Duration Gamma-Ray Bursts. ApJ, 670:1254-1259, December 2007. doi:10.1086/522195

E. Berger. Short-Duration Gamma-Ray Bursts. ARA\&A, 52: 43-105, August 2014. doi:10.1146/annurev-astro-081913-035926

M. G. Bernardini, C. L. Bianco, L. Caito, P. Chardonnet, A. Corsi, M. G. Dainotti, F. Fraschetti, R. Guida, R. Ruffini, and S. S. Xue. GRB970228 as a prototype for short GRBs with afterglow. Nuovo Cimento B Serie, 121:1439-1440, December 2006. doi:10.1393/ncb/i2007-10283-0

M. G. Bernardini, C. L. Bianco, L. Caito, M. G. Dainotti,

R. Guida, and R. Ruffini. GRB 970228 and a class of GRBs with an initial spikelike emission. A\&SA, 474:L13-L16, October 2007. doi:10.1051/0004-6361:20078300

M. G. Bernardini, R. Margutti, J. Mao, E. Zaninoni, and G. Chincarini. The X-ray light curve of gamma-ray bursts: clues to the central engine. A\&AA, 539:A3, March 2012a. doi:10.1051/0004-6361/201117895

M. G. Bernardini, R. Margutti, E. Zaninoni, and G. Chincarini. A universal scaling for short and long gamma-ray bursts: $\mathrm{E}_{X, i s o}-\mathrm{E}_{, i s o}-\mathrm{E}_{p k}$. MNRAS, 425:1199-1204, September 2012b. doi:10.1111/j.1365-2966.2012.21487.x
M. Betoule, R. Kessler, J. Guy, J. Mosher, D. Hardin, R. Biswas, P. Astier, P. El-Hage, M. Konig, S. Kuhlmann, J. Marriner, R. Pain, N. Regnault, C. Balland, B. A. Bassett, P. J. Brown, H. Campbell, R. G. Carlberg, F. Cellier-Holzem, D. Cinabro, A. Conley, C. B. D'Andrea, D. L. DePoy, M. Doi, R. S. Ellis, S. Fabbro, A. V. Filippenko, R. J. Foley, J. A. Frieman, D. Fouchez, L. Galbany, A. Goobar, R. R. Gupta, G. J. Hill, R. Hlozek, C. J. Hogan, I. M. Hook, D. A. Howell, S. W. Jha, L. Le Guillou, G. Leloudas, C. Lidman, J. L. Marshall, A. Möller, A. M. Mourão, J. Neveu, R. Nichol, M. D. Olmstead, N. Palanque-Delabrouille, S. Perlmutter, J. L. Prieto, C. J. Pritchet, M. Richmond, A. G. Riess, V. Ruhlmann-Kleider, M. Sako, K. Schahmaneche, D. P. Schneider, M. Smith, J. Sollerman, M. Sullivan, N. A. Walton, and C. J. Wheeler. Improved cosmological constraints from a joint analysis of the SDSS-II and SNLS supernova samples. A\&BA, 568:A22, August 2014. doi:10.1051/0004-6361/201423413

J. S. Bloom, D. A. Frail, and R. Sari. The Prompt Energy Release of Gamma-Ray Bursts using a Cosmological k-Correction. AJ, 121:2879-2888, June 2001. doi:10.1086/321093

M. Boër and B. Gendre. Evidences for two Gamma-Ray Burst afterglow emission regimes. AESA, 361:L21-L24, September 2000 .

N. R. Butler, J. S. Bloom, and D. Poznanski. The Cosmic Rate, Luminosity Function, and Intrinsic Correlations of Long Gamma-Ray Bursts. ApJ, 711:495-516, March 2010. doi:10.1088/0004-637X/711/1/495

L. Caito, M. G. Bernardini, C. L. Bianco, M. G. Dainotti, R. Guida, and R. Ruffini. GRB060614: a "fake" short GRB from a merging binary system. A\&SA, 498:501-507, May 2009. doi:10.1051/0004-6361/200810676

J. Calcino and T. Davis. The need for accurate redshifts in supernova cosmology. Journal of Cosmology and Astroparticle Physics, 1:038, January 2017. doi:10.1088/1475-7516/2017/01/038

J. K. Cannizzo and N. Gehrels. A New Paradigm for Gamma-ray Bursts: Long-term Accretion Rate Modulation by an External Accretion Disk. ApJ, 700:1047-1058, August 2009. doi:10.1088/0004-637X/700/2/1047.

J. K. Cannizzo, E. Troja, and N. Gehrels. Fall-back Disks in Long and Short Gamma-Ray Bursts. ApJ, 734:35, June 2011. doi:10.1088/0004-637X/734/1/35.

Z. Cano. Gamma-Ray Burst Supernovae as Standardizable Candles. ApJ, 794:121, October 2014. doi:10.1088/0004-637X/794/2/121

V. F. Cardone, S. Capozziello, and M. G. Dainotti. An updated gamma-ray bursts Hubble diagram. MNRAS, 400:775-790, December 2009. doi:10.1111/j.1365-2966.2009.15456.x

V. F. Cardone, M. G. Dainotti, S. Capozziello, and R. Willingale. Constraining cosmological parameters by gamma-ray burst X-ray afterglow light curves. MNRAS, 408:1181-1186, October 2010. doi:10.1111/j.1365-2966.2010.17197.x. 
S. B. Cenko, J. Kelemen, F. A. Harrison, D. B. Fox, S. R. Kulkarni, M. M. Kasliwal, E. O. Ofek, A. Rau, A. Gal-Yam, D. A. Frail, and D.-S. Moon. Dark Bursts in the Swift Era: The Palomar 60 Inch-Swift Early Optical Afterglow Catalog. ApJ, 693:1484-1493, March 2009. doi:10.1088/0004-637X/693/2/1484

M. Chevallier and D. Polarski. Accelerating Universes with Scaling Dark Matter. International Journal of Modern Physics D, 10:213-223, 2001. doi:10.1142/S0218271801000822.

G. Chincarini, A. Moretti, P. Romano, A. D. Falcone, D. Morris, J. Racusin, S. Campana, S. Covino, C. Guidorzi,

G. Tagliaferri, D. N. Burrows, C. Pagani, M. Stroh, D. Grupe, M. Capalbi, G. Cusumano, N. Gehrels, P. Giommi, V. La Parola, V. Mangano, T. Mineo, J. A. Nousek, P. T. O'Brien, K. L. Page, M. Perri, E. Troja, R. Willingale, and B. Zhang. The First Survey of X-Ray Flares from Gamma-Ray Bursts Observed by Swift: Temporal Properties and Morphology. ApJ, 671:1903-1920, December 2007. doi:10.1086/521591

A. Cucchiara, A. J. Levan, D. B. Fox, N. R. Tanvir, T. N. Ukwatta, E. Berger, T. Krühler, A. Küpcü Yoldaş, X. F. Wu, K. Toma, J. Greiner, F. E. Olivares, A. Rowlinson, L. Amati, T. Sakamoto, K. Roth, A. Stephens, A. Fritz, J. P. U. Fynbo, J. Hjorth, D. Malesani, P. Jakobsson, K. Wiersema, P. T. O'Brien, A. M. Soderberg, R. J. Foley, A. S. Fruchter, J. Rhoads, R. E. Rutledge, B. P. Schmidt, M. A. Dopita, P. Podsiadlowski, R. Willingale, C. Wolf, S. R. Kulkarni, and P. D'Avanzo. A Photometric Redshift of $z \sim 9.4$ for GRB 090429B. ApJ, 736:7, July 2011. doi:10.1088/0004-637X/736/1/7

G. D'Agostini. Fits, and especially linear fits, with errors on both axes, extra variance of the data points and other complications. ArXiv Physics e-prints, November 2005.

Z. G. Dai and T. Lu. Gamma-ray burst afterglows and evolution of postburst fireballs with energy injection from strongly magnetic millisecond pulsars. A\&SA, 333:L87-L90, May 1998.

M. Dainotti, V. Petrosian, R. Willingale, P. O'Brien, M. Ostrowski, and S. Nagataki. Luminosity-time and luminosity-luminosity correlations for GRB prompt and afterglow plateau emissions. MNRAS, 451:3898-3908, August 2015a. doi:10.1093/mnras/stv1229

M. Dainotti, R. Del Vecchio, and M. Tarnopolski. Gamma Ray Burst Prompt correlations. ArXiv e-prints, December 2016a.

M. G. Dainotti, M. G. Bernardini, C. L. Bianco, L. Caito, R. Guida, and R. Ruffini. GRB 060218 and GRBs associated with supernovae Ib/c. A\&A , 471:L29-L32, August 2007. doi:10.1051/0004-6361:20078068.

M. G. Dainotti, V. F. Cardone, and S. Capozziello. A time-luminosity correlation for $\gamma$-ray bursts in the X-rays. MNRAS, 391:L79-L83, November 2008. doi:10.1111/j.1745-3933.2008.00560.x

M. G. Dainotti, R. Willingale, S. Capozziello, V. Fabrizio Cardone, and M. Ostrowski. Discovery of a Tight Correlation for Gamma-ray Burst Afterglows with "Canonical" Light Curves. ApJL, 722:L215-L219, October 2010. doi:10.1088/2041-8205/722/2/L215

M. G. Dainotti, V. Fabrizio Cardone, S. Capozziello, M. Ostrowski, and R. Willingale. Study of Possible Systematics in the $\mathrm{L}^{*}{ }_{X}-\mathrm{T}^{*}{ }_{a}$ Correlation of Gamma-ray Bursts. ApJ, 730:135, April 2011a. doi:10.1088/0004-637X/730/2/135

M. G. Dainotti, M. Ostrowski, and R. Willingale. Towards a standard gamma-ray burst: tight correlations between the prompt and the afterglow plateau phase emission. MNRAS, 418:2202-2206, December 2011b. doi:10.1111/j.1365-2966.2011.19433.x
M. G. Dainotti, V. F. Cardone, E. Piedipalumbo, and S. Capozziello. Slope evolution of GRB correlations and cosmology. MNRAS, 436:82-88, November 2013a. doi:10.1093/mnras/stt1516

M. G. Dainotti, V. Petrosian, J. Singal, and M. Ostrowski. Determination of the Intrinsic Luminosity Time Correlation in the X-Ray Afterglows of Gamma-Ray Bursts. ApJ, 774:157, September 2013b. doi:10.1088/0004-637X/774/2/157.

M. G. Dainotti, R. Del Vecchio, S. Nagataki, and S. Capozziello. Selection Effects in Gamma-Ray Burst Correlations:

Consequences on the Ratio between Gamma-Ray Burst and Star Formation Rates. ApJ, 800:31, February 2015b. doi:10.1088/0004-637X/800/1/31.

M. G. Dainotti, S. Nagataki, K. Maeda, S. Postnikov, and E. Pian. A study of gamma ray bursts with afterglow plateau phases associated with supernovae. ArXiv e-prints, December 2016b.

M. G. Dainotti, S. Postnikov, X. Hernandez, and M. Ostrowski. A Fundamental Plane for Long Gamma-Ray Bursts with X-Ray Plateaus. ApJL, 825:L20, July 2016c. doi:10.3847/2041-8205/825/2/L20

S. Dall'Osso, G. Stratta, D. Guetta, S. Covino, G. De Cesare, and L. Stella. Gamma-ray bursts afterglows with energy injection from a spinning down neutron star. $A \& A$, 526:A121, February 2011. doi:10.1051/0004-6361/201014168

P. D'Avanzo, R. Salvaterra, B. Sbarufatti, L. Nava, A. Melandri, M. G. Bernardini, S. Campana, S. Covino, D. Fugazza, G. Ghirlanda, G. Ghisellini, V. L. Parola, M. Perri, S. D. Vergani, and G. Tagliaferri. A complete sample of bright Swift Gamma-ray bursts: X-ray afterglow luminosity and its correlation with the prompt emission. MNRAS, 425:506-513, September 2012. doi:10.1111/j.1365-2966.2012.21489.x

T. M. Davis, E. Mörtsell, J. Sollerman, A. C. Becker, S. Blondin, P. Challis, A. Clocchiatti, A. V. Filippenko, R. J. Foley, P. M. Garnavich, S. Jha, K. Krisciunas, R. P. Kirshner,

B. Leibundgut, W. Li, T. Matheson, G. Miknaitis, G. Pignata, A. Rest, A. G. Riess, B. P. Schmidt, R. C. Smith, J. Spyromilio, C. W. Stubbs, N. B. Suntzeff, J. L. Tonry, W. M. Wood-Vasey, and A. Zenteno. Scrutinizing Exotic Cosmological Models Using ESSENCE Supernova Data Combined with Other Cosmological Probes. ApJ, 666: 716-725, September 2007. doi:10.1086/519988.

R. Del Vecchio, M. Giovanna Dainotti, and M. Ostrowski. Study of GRB Light-curve Decay Indices in the Afterglow Phase. ApJ, 828:36, September 2016. doi:10.3847/0004-637X/828/1/36.

P. C. Duffell and A. I. MacFadyen. From Engine to Afterglow: Collapsars Naturally Produce Top-heavy Jets and Early-time Plateaus in Gamma-Ray Burst Afterglows. ApJ, 806:205, June 2015. doi:10.1088/0004-637X/806/2/205

B. Efron and V. Petrosian. A simple test of independence for truncated data with applications to redshift surveys. ApJ, 399:345-352, November 1992. doi:10.1086/171931

E. E. Fenimore, J. J. M. in 't Zand, J. P. Norris, J. T. Bonnell, and R. J. Nemiroff. Gamma-Ray Burst Peak Duration as a Function of Energy. ApJL, 448:L101, August 1995. doi: $10.1086 / 309603$

C. Firmani, G. Ghisellini, V. Avila-Reese, and G. Ghirlanda. Discovery of a tight correlation among the prompt emission properties of long gamma-ray bursts. MNRAS, 370:185-197, July 2006. doi:10.1111/j.1365-2966.2006.10445.x 
N. Gehrels, S. D. Barthelmy, D. N. Burrows, J. K. Cannizzo, G. Chincarini, E. Fenimore, C. Kouveliotou, P. O'Brien, D. M. Palmer, J. Racusin, P. W. A. Roming, T. Sakamoto, J. Tueller, R. A. M. J. Wijers, and B. Zhang. Correlations of Prompt and Afterglow Emission in Swift Long and Short Gamma-Ray Bursts. ApJ, 689:1161-1172, December 2008. doi:10.1086/592766

Neil Gehrels. Short GRB Prompt and Afterglow Correlations. 2007.

G. Ghirlanda. Advances on GRB as cosmological tools. In G. Giobbi, A. Tornambe, G. Raimondo, M. Limongi, L. A. Antonelli, N. Menci, and E. Brocato, editors, American Institute of Physics Conference Series, volume 1111 of American Institute of Physics Conference Series, pages 579-586, May 2009. doi:10.1063/1.3141613

G. Ghirlanda, G. Ghisellini, and D. Lazzati. The Collimation-corrected Gamma-Ray Burst Energies Correlate with the Peak Energy of Their $\nu \mathrm{F}_{n u}$ Spectrum. ApJ, 616: 331-338, November 2004. doi:10.1086/424913

G. Ghirlanda, G. Ghisellini, and C. Firmani. Gamma-ray bursts as standard candles to constrain the cosmological parameters. New Journal of Physics, 8:123, July 2006. doi:10.1088/1367-2630/8/7/123.

G. Ghisellini, M. Nardini, G. Ghirlanda, and A. Celotti. A unifying view of gamma-ray burst afterglows. MNRAS, 393: 253-271, February 2009. doi:10.1111/j.1365-2966.2008.14214.x

B. P. Gompertz, P. T. O'Brien, G. A. Wynn, and A. Rowlinson. Can magnetar spin-down power extended emission in some short GRBs? MNRAS, 431:1745-1751, May 2013. doi:10.1093/mnras/stt293

D. Grupe, J. A. Nousek, P. Veres, B.-B. Zhang, and N. Gehrels. Evidence for New Relations between Gamma-Ray Burst Prompt and X-Ray Afterglow Emission from 9 Years of Swift. ApJS, 209:20, December 2013. doi:10.1088/0067-0049/209/2/20

R. Guida, M. G. Bernardini, C. L. Bianco, L. Caito, M. G. Dainotti, and R. Ruffini. The Amati relation in the "fireshell" model. $A \& 3 A, 487:$ L37-L40, August 2008. doi:10.1051/0004-6361:200810338

R. Hascoët, F. Daigne, and R. Mochkovitch. The prompt-early afterglow connection in gamma-ray bursts: implications for the early afterglow physics. MNRAS, 442:20-27, July 2014. doi:10.1093/mnras/stu750

J. Heise, J. I. Zand, R. M. Kippen, and P. M. Woods. X-Ray Flashes and X-Ray Rich Gamma Ray Bursts. In E. Costa, F. Frontera, and J. Hjorth, editors, Gamma-ray Bursts in the Afterglow Era, page 16, 2001. doi:10.1007/108538534

H. Ito, S. Nagataki, J. Matsumoto, S.-H. Lee, A. Tolstov, J. Mao, M. Dainotti, and A. Mizuta. Spectral and Polarization Properties of Photospheric Emission from Stratified Jets. $A p J, 789: 159$, July 2014. doi:10.1088/0004-637X/789/2/159

P. Jakobsson, J. Hjorth, J. P. U. Fynbo, D. Watson, K. Pedersen, G. Björnsson, and J. Gorosabel. Swift Identification of Dark Gamma-Ray Bursts. ApJL, 617: L21-L24, December 2004. doi:10.1086/427089

Y. Kaneko, E. Ramirez-Ruiz, J. Granot, C. Kouveliotou, S. E. Woosley, S. K. Patel, E. Rol, J. J. M. in 't Zand, A. J. van der Horst, R. A. M. J. Wijers, and R. Strom. Prompt and Afterglow Emission Properties of Gamma-Ray Bursts with Spectroscopically Identified Supernovae. ApJ, 654:385-402, January 2007. doi:10.1086/508324
D. A. Kann, S. Klose, B. Zhang, D. Malesani, E. Nakar, A. Pozanenko, A. C. Wilson, N. R. Butler, P. Jakobsson, S. Schulze, M. Andreev, L. A. Antonelli, I. F. Bikmaev, V. Biryukov, M. Böttcher, R. A. Burenin, J. M. Castro Cerón, A. J. Castro-Tirado, G. Chincarini, B. E. Cobb, S. Covino, P. D'Avanzo, V. D'Elia, M. Della Valle, A. de Ugarte Postigo, Y. Efimov, P. Ferrero, D. Fugazza, J. P. U. Fynbo, M. Gålfalk, F. Grundahl, J. Gorosabel, S. Gupta, S. Guziy, B. Hafizov, J. Hjorth, K. Holhjem, M. Ibrahimov, M. Im, G. L. Israel, M. Jelinek, B. L. Jensen, R. Karimov, I. M. Khamitov, Ü. Kiziloğlu, E. Klunko, P. Kubánek, A. S. Kutyrev, P. Laursen, A. J. Levan, F. Mannucci, C. M. Martin, A. Mescheryakov, N. Mirabal, J. P. Norris, J.-E. Ovaldsen, D. Paraficz, E. Pavlenko, S. Piranomonte, A. Rossi, V. Rumyantsev, R. Salinas, A. Sergeev, D. Sharapov, J. Sollerman, B. Stecklum, L. Stella, G. Tagliaferri, N. R. Tanvir, J. Telting, V. Testa, A. C. Updike, A. Volnova, D. Watson, K. Wiersema, and D. Xu. The Afterglows of Swift-era Gamma-ray Bursts. I. Comparing pre-Swift and Swift-era Long/Soft (Type II) GRB Optical Afterglows. ApJ, 720:1513-1558, September 2010. doi:10.1088/0004-637X/720/2/1513

D. Kazanas, J. L. Racusin, J. Sultana, and A. Mastichiadis. The Statistics of the Prompt-to-Afterglow GRB Flux Ratios and the Supercritical Pile GRB Model. ArXiv e-prints, January 2015.

R. M. Kippen, P. M. Woods, J. Heise, J. I. Zand, R. D. Preece, and M. S. Briggs. BATSE Observations of Fast X-Ray Transients Detected by BeppoSAX-WFC. In E. Costa, F. Frontera, and J. Hjorth, editors, Gamma-ray Bursts in the Afterglow Era, page 22, 2001. doi:10.1007/108538535

R. W. Klebesadel, I. B. Strong, and R. A. Olson. Observations of Gamma-Ray Bursts of Cosmic Origin. ApJL, 182:L85, June 1973. doi:10.1086/181225

S. Kobayashi, T. Piran, and R. Sari. Can Internal Shocks Produce the Variability in Gamma-Ray Bursts? ApJ, 490:92, November 1997. doi:10.1086/512791

Y. Kodama, D. Yonetoku, T. Murakami, S. Tanabe, R. Tsutsui, and T. Nakamura. Gamma-ray bursts in 1.8 ; z ; 5.6 suggest that the time variation of the dark energy is small. MNRAS, 391:L1-L4, November 2008. doi:10.1111/j.1745-3933.2008.00508.x

E. Komatsu, K. M. Smith, J. Dunkley, C. L. Bennett, B. Gold, G. Hinshaw, N. Jarosik, D. Larson, M. R. Nolta, L. Page, D. N. Spergel, M. Halpern, R. S. Hill, A. Kogut, M. Limon, S. S. Meyer, N. Odegard, G. S. Tucker, J. L. Weiland, E. Wollack, and E. L. Wright. Seven-year Wilkinson Microwave Anisotropy Probe (WMAP) Observations: Cosmological Interpretation. ApJS, 192:18, February 2011. doi:10.1088/0067-0049/192/2/18

C. Kouveliotou, C. A. Meegan, G. J. Fishman, N. P. Bhat, M. S. Briggs, T. M. Koshut, W. S. Paciesas, and G. N. Pendleton. Identification of two classes of gamma-ray bursts. ApJL, 413: L101-L104, August 1993. doi:10.1086/186969

P. Kumar, R. Narayan, and J. L. Johnson. Properties of Gamma-Ray Burst Progenitor Stars. Science, 321:376-, July 2008. doi:10.1126/science.1159003.

K. Leventis, R. A. M. J. Wijers, and A. J. van der Horst. The plateau phase of gamma-ray burst afterglows in the thick-shell scenario. MNRAS, 437:2448-2460, January 2014. doi: $10.1093 / \mathrm{mnras} / \mathrm{stt} 2055$

L. Li, E.-W. Liang, Q.-W. Tang, J.-M. Chen, S.-Q. Xi, H.-J. Lü, H. Gao, B. Zhang, J. Zhang, S.-X. Yi, R.-J. Lu, L.-Z. Lü, and J.-Y. Wei. A Comprehensive Study of Gamma-Ray Burst Optical Emission. I. Flares and Early Shallow-decay Component. ApJ, 758:27, October 2012. doi:10.1088/0004-637X/758/1/27. 
X. Li and J. Hjorth. Light Curve Properties of Supernovae Associated With Gamma-ray Bursts. ArXiv e-prints, July 2014.

E. Liang and B. Zhang. Identification of Two Categories of Optically Bright Gamma-Ray Bursts. ApJL, 638:L67-L70, February 2006a. doi:10.1086/501049

E. Liang and B. Zhang. Calibration of gamma-ray burst luminosity indicators. MNRAS, 369:L37-L41, June 2006b. doi:10.1111/j.1745-3933.2006.00169.x

E.-W. Liang, B.-B. Zhang, and B. Zhang. A Comprehensive Analysis of Swift XRT Data. II. Diverse Physical Origins of the Shallow Decay Segment. ApJ, 670:565-583, November 2007. doi:10.1086/521870

E.-W. Liang, S.-X. Yi, J. Zhang, H.-J. Lü, B.-B. Zhang, and B. Zhang. Constraining Gamma-ray Burst Initial Lorentz Factor with the Afterglow Onset Feature and Discovery of a Tight $\Gamma_{0}-\mathrm{E}_{\text {gamma,iso }}$ Correlation. ApJ, 725:2209-2224, December 2010. doi:10.1088/0004-637X/725/2/2209

N. Liang, W. K. Xiao, Y. Liu, and S. N. Zhang. A Cosmology-Independent Calibration of Gamma-Ray Burst Luminosity Relations and the Hubble Diagram. ApJ, 685: 354-360, September 2008. doi:10.1086/590903.

C. C. Lindner, M. Milosavljević, S. M. Couch, and P. Kumar. Collapsar Accretion and the Gamma-Ray Burst X-Ray Light Curve. ApJ, 713:800-815, April 2010. doi:10.1088/0004-637X/713/2/800

N. M. Lloyd and V. Petrosian. Distribution of Spectral Characteristics and the Cosmological Evolution of Gamma-Ray Bursts. ApJ, 511:550-561, February 1999. doi:10.1086/306719

N. M. Lloyd, V. Petrosian, and R. D. Preece. Synchrotron emission as the source of GRB spectra, Part II: Observations. In R. M. Kippen, R. S. Mallozzi, and G. J. Fishman, editors, Gamma-ray Bursts, 5th Huntsville Symposium, volume 526 of American Institute of Physics Conference Series, pages 155-159, September 2000. doi:10.1063/1.1361525

H.-J. Lü and B. Zhang. A Test of the Millisecond Magnetar Central Engine Model of Gamma-Ray Bursts with Swift Data. ApJ, 785:74, April 2014. doi:10.1088/0004-637X/785/1/74.

N. Lyons, P. T. O'Brien, B. Zhang, R. Willingale, E. Troja, and R. L. C. Starling. Can X-ray emission powered by a spinning-down magnetar explain some gamma-ray burst light-curve features? MNRAS, 402:705-712, February 2010. doi:10.1111/j.1365-2966.2009.15538.x

V. Mangano, B. Sbarufatti, and G. Stratta. Extending the plateau luminosity-duration anticorrelation. Memorie della Societa Astronomica Italiana Supplementi, 21:143, 2012.

R. Margutti, C. Guidorzi, G. Chincarini, M. G. Bernardini, F. Genet, J. Mao, and F. Pasotti. Lag-luminosity relation in $\gamma$-ray burst X-ray flares: a direct link to the prompt emission. MNRAS, 406:2149-2167, August 2010. doi:10.1111/j.1365-2966.2010.16824.x

R. Margutti, E. Zaninoni, M. G. Bernardini, G. Chincarini, F. Pasotti, C. Guidorzi, L. Angelini, D. N. Burrows, M. Capalbi, P. A. Evans, N. Gehrels, J. Kennea, V. Mangano, A. Moretti, J. Nousek, J. P. Osborne, K. L. Page, M. Perri, J. Racusin, P. Romano, B. Sbarufatti, S. Stafford, and M. Stamatikos. The prompt-afterglow connection in gamma-ray bursts: a comprehensive statistical analysis of Swift X-ray light curves. MNRAS, 428:729-742, January 2013. doi:10.1093/mnras/sts066

A. Maxham and B. Zhang. Modeling Gamma-Ray Burst X-Ray Flares Within the Internal Shock Model. ApJ, 707:1623-1633, December 2009. doi:10.1088/0004-637X/707/2/1623
E. P. Mazets, S. V. Golenetskii, V. N. Ilyinskii, V. N. Panov, R. L. Aptekar, Y. A. Guryan, M. P. Proskura, I. A. Sokolov, Z. Y. Sokolova, T. V. Kharitonova, A. V. Dyatchkov, and N. G. Khavenson. Catalog of cosmic gamma-ray bursts from the KONUS experiment data. Ap\&SS, 80:85-117, November 1981. doi:10.1007/BF00649141.

A. Melandri, C. G. Mundell, S. Kobayashi, C. Guidorzi, A. Gomboc, I. A. Steele, R. J. Smith, D. Bersier, C. J. Mottram, D. Carter, M. F. Bode, P. T. O'Brien, N. R. Tanvir, E. Rol, and R. Chapman. The Early-Time Optical Properties of Gamma-Ray Burst Afterglows. ApJ, 686:1209-1230, October 2008. doi:10.1086/591243

A. Melandri, S. Covino, D. Rogantini, R. Salvaterra, B. Sbarufatti, M. G. Bernardini, S. Campana, P. D'Avanzo, V. D'Elia, D. Fugazza, G. Ghirlanda, G. Ghisellini, L. Nava, S. D. Vergani, and G. Tagliaferri. Optical and X-ray rest-frame light curves of the BAT6 sample. $A \& A$, 565:A72, May 2014. doi:10.1051/0004-6361/201323361

P. Mészáros. Theoretical models of gamma-ray bursts. In C. A. Meegan, R. D. Preece, and T. M. Koshut, editors,

Gamma-Ray Bursts, 4th Hunstville Symposium, volume 428 of American Institute of Physics Conference Series, pages 647-656, May 1998. doi:10.1063/1.55394

P. Mészáros. Gamma-ray bursts. Reports on Progress in Physics, 69:2259-2321, August 2006. doi:10.1088/0034-4885/69/8/R01

B. D. Metzger, D. Giannios, T. A. Thompson, N. Bucciantini, and E. Quataert. The protomagnetar model for gamma-ray bursts. MNRAS, 413:2031-2056, May 2011. doi:10.1111/j.1365-2966.2011.18280.x

M. Nardini, G. Ghisellini, G. Ghirlanda, F. Tavecchio, C. Firmani, and D. Lazzati. Clustering of the optical-afterglow luminosities of long gamma-ray bursts. $A \mathscr{E} A, 451: 821-833$, June 2006. doi:10.1051/0004-6361:20054085

M. Nardini, G. Ghisellini, and G. Ghirlanda. Optical afterglow luminosities in the Swift epoch: confirming clustering and bimodality. MNRAS, 386:L87-L91, May 2008a. doi:10.1111/j.1745-3933.2008.00467.x

M. Nardini, G. Ghisellini, and G. Ghirlanda. Optical afterglows of gamma-ray bursts: a bimodal distribution? MNRAS, 383: 1049-1057, January 2008b. doi:10.1111/j.1365-2966.2007.12588.x

J. P. Norris and J. T. Bonnell. Short Gamma-Ray Bursts with Extended Emission. ApJ, 643:266-275, May 2006. doi:10.1086/502796.

J. A. Nousek, C. Kouveliotou, D. Grupe, K. L. Page, J. Granot, E. Ramirez-Ruiz, S. K. Patel, D. N. Burrows, V. Mangano, S. Barthelmy, A. P. Beardmore, S. Campana, M. Capalbi, G. Chincarini, G. Cusumano, A. D. Falcone, N. Gehrels, P. Giommi, M. R. Goad, O. Godet, C. P. Hurkett, J. A. Kennea, A. Moretti, P. T. O'Brien, J. P. Osborne, P. Romano, G. Tagliaferri, and A. A. Wells. Evidence for a Canonical Gamma-Ray Burst Afterglow Light Curve in the Swift XRT Data. ApJ, 642:389-400, May 2006. doi:10.1086/500724

M. Nysewander, A. S. Fruchter, and A. Pe'er. A Comparison of the Afterglows of Short- and Long-duration Gamma-ray Bursts. ApJ, 701:824-836, August 2009. doi:10.1088/0004-637X/701/1/824

S. R. Oates, M. J. Page, P. Schady, M. de Pasquale, T. S. Koch, A. A. Breeveld, P. J. Brown, M. M. Chester, S. T. Holland, E. A. Hoversten, N. P. M. Kuin, F. E. Marshall, P. W. A. Roming, M. Still, D. E. vanden Berk, S. Zane, and J. A. Nousek. A statistical study of gamma-ray burst afterglows measured by the Swift Ultraviolet Optical Telescope. MNRAS, 395:490-503, May 2009. doi:10.1111/j.1365-2966.2009.14544.x 
S. R. Oates, M. J. Page, M. De Pasquale, P. Schady, A. A. Breeveld, S. T. Holland, N. P. M. Kuin, and F. E. Marshall. A correlation between the intrinsic brightness and average decay rate of Swift/UVOT gamma-ray burst optical/ultraviolet light curves. MNRAS, 426:L86-L90, October 2012. doi:10.1111/j.1745-3933.2012.01331.x

S. R. Oates, J. L. Racusin, M. De Pasquale, M. J. Page, A. J. Castro-Tirado, J. Gorosabel, P. J. Smith, A. A. Breeveld, and N. P. M. Kuin. Exploring the canonical behaviour of long gamma-ray bursts using an intrinsic multiwavelength afterglow correlation. MNRAS, 453:4121-4135, November 2015. doi: $10.1093 / \mathrm{mnras} / \mathrm{stv} 1956$

P. T. O'Brien, R. Willingale, J. Osborne, M. R. Goad, K. L. Page, S. Vaughan, E. Rol, A. Beardmore, O. Godet, C. P. Hurkett, A. Wells, B. Zhang, S. Kobayashi, D. N. Burrows, J. A. Nousek, J. A. Kennea, A. Falcone, D. Grupe, N. Gehrels, S. Barthelmy, J. Cannizzo, J. Cummings, J. E. Hill, H. Krimm, G. Chincarini, G. Tagliaferri, S. Campana, A. Moretti, P. Giommi, M. Perri, V. Mangano, and V. LaParola. The Early X-Ray Emission from GRBs. ApJ, 647:1213-1237, August 2006. doi:10.1086/505457

A. Panaitescu and W. T. Vestrand. Taxonomy of gamma-ray burst optical light curves: identification of a salient class of early afterglows. MNRAS, 387:497-504, June 2008. doi:10.1111/j.1365-2966.2008.13231.x

A. Panaitescu and W. T. Vestrand. Optical afterglows of gamma-ray bursts: peaks, plateaus and possibilities. MNRAS, 414:3537-3546, July 2011. doi:10.1111/j.1365-2966.2011.18653.x

S. Perlmutter, G. Aldering, M. della Valle, S. Deustua, R. S. Ellis, S. Fabbro, A. Fruchter, G. Goldhaber, D. E. Groom, I. M. Hook, A. G. Kim, M. Y. Kim, R. A. Knop, C. Lidman, R. G. McMahon, P. Nugent, R. Pain, N. Panagia, C. R. Pennypacker, P. Ruiz-Lapuente, B. Schaefer, and N. Walton. Discovery of a supernova explosion at half the age of the universe. Nature, 391:51, January 1998. doi:10.1038/34124

R. Perna, P. J. Armitage, and B. Zhang. Flares in Long and Short Gamma-Ray Bursts: A Common Origin in a Hyperaccreting Accretion Disk. ApJL, 636:L29-L32, January 2006. doi:10.1086/499775

J. H. Petersen, K. K. Holst, and E. Budtz-Jørgensen. Correcting a Statistical Artifact in the Estimation of the Hubble Constant Based on Type Ia Supernovae Results in a Change in Estimate of $1.2 \%$. ApJ, 723:966-968, November 2010. doi:10.1088/0004-637X/723/1/966

V. Petrosian, A. Bouvier, and F. Ryde. Gamma-Ray Bursts as Cosmological Tools. ArXiv e-prints, September 2009.

Vahe Petrosian, Jack Singal, and Lukasz Stawarz. Luminosity correlations, luminosity evolutions, and radio loudness of agns from multiwavelength observations. In Multiwavelength AGN Surveys and Studies, volume 9 of Proceedings of the International Astronomical Union, pages 172-172, 102013. doi:10.1017/S174392131400369X URL http:

//journals.cambridge.org/article_S174392131400369x.

M. M. Phillips. The absolute magnitudes of Type IA supernovae. ApJL, 413:L105-L108, August 1993. doi:10.1086/186970

Planck Collaboration, P. A. R. Ade, N. Aghanim, M. Arnaud, M. Ashdown, J. Aumont, C. Baccigalupi, A. J. Banday, R. B. Barreiro, J. G. Bartlett, and et al. Planck 2015 results. XIII. Cosmological parameters. A\&SA, 594:A13, September 2016. doi:10.1051/0004-6361/201525830

S. Postnikov, M. G. Dainotti, X. Hernandez, and S. Capozziello. Nonparametric Study of the Evolution of the Cosmological Equation of State with SNeIa, BAO, and High-redshift GRBs. ApJ, 783:126, March 2014. doi:10.1088/0004-637X/783/2/126.
D. Proga and B. Zhang. The late time evolution of gamma-ray bursts: ending hyperaccretion and producing flares. MNRAS, 370:L61-L65, July 2006. doi:10.1111/j.1745-3933.2006.00189.x

J. L. Racusin, S. R. Oates, P. Schady, D. N. Burrows, M. de Pasquale, D. Donato, N. Gehrels, S. Koch, J. McEnery, T. Piran, P. Roming, T. Sakamoto, C. Swenson, E. Troja, V. Vasileiou, F. Virgili, D. Wanderman, and B. Zhang. Fermi and Swift Gamma-ray Burst Afterglow Population Studies. ApJ, 738:138, September 2011. doi:10.1088/0004-637X/738/2/138

J. L. Racusin, S. R. Oates, M. de Pasquale, and D. Kocevski. A Correlation between the Intrinsic Brightness and Average Decay Rate of Gamma-Ray Burst X-Ray Afterglow Light Curves. ApJ, 826:45, July 2016. doi:10.3847/0004-637X/826/1/45

N. Rea, M. Gullon, J. A. Pons, R. Perna, M. G. Dainotti, J. A. Miralles, and D. F. Torres. Constraining the GRB-magnetar model by means of the Galactic pulsar population. ArXiv e-prints, October 2015.

M. J. Rees and P. Mészáros. Refreshed Shocks and Afterglow Longevity in Gamma-Ray Bursts. ApJL, 496:L1-L4, March 1998. doi:10.1086/311244

A. G. Riess, A. V. Filippenko, P. Challis, A. Clocchiatti, A. Diercks, P. M. Garnavich, R. L. Gilliland, C. J. Hogan, S. Jha, R. P. Kirshner, B. Leibundgut, M. M. Phillips, D. Reiss, B. P. Schmidt, R. A. Schommer, R. C. Smith, J. Spyromilio, C. Stubbs, N. B. Suntzeff, and J. Tonry. Observational Evidence from Supernovae for an Accelerating Universe and a Cosmological Constant. AJ, 116:1009-1038, September 1998. doi:10.1086/300499

A. G. Riess, L. Macri, S. Casertano, M. Sosey, H. Lampeitl, H. C. Ferguson, A. V. Filippenko, S. W. Jha, W. Li, R. Chornock, and D. Sarkar. A Redetermination of the Hubble Constant with the Hubble Space Telescope from a Differential Distance Ladder. ApJ, 699:539-563, July 2009. doi:10.1088/0004-637X/699/1/539

S. A. Rodney, A. G. Riess, D. M. Scolnic, D. O. Jones, S. Hemmati, A. Molino, C. McCully, B. Mobasher, L.-G. Strolger, O. Graur, B. Hayden, and S. Casertano. Two SNe Ia at Redshift 2: Improved Classification and Redshift Determination with Medium-band Infrared Imaging. $A J, 150$ : 156, November 2015. doi:10.1088/0004-6256/150/5/156

A. Rowlinson and P. O'Brien. Energy injection in short GRBs and the role of magnetars. In Gamma-Ray Bursts 2012 Conference (GRB 2012), 2012.

A. Rowlinson, P. T. O'Brien, B. D. Metzger, N. R. Tanvir, and A. J. Levan. Signatures of magnetar central engines in short GRB light curves. MNRAS, 430:1061-1087, April 2013. doi:10.1093/mnras/sts683

A. Rowlinson, B. P. Gompertz, M. Dainotti, P. T. O'Brien, R. A. M. J. Wijers, and A. J. van der Horst. Constraining properties of GRB magnetar central engines using the observed plateau luminosity and duration correlation. MNRAS, 443: 1779-1787, September 2014. doi:10.1093/mnras/stu1277

R. Ruffini, M. Muccino, C. L. Bianco, M. Enderli, L. Izzo, M. Kovacevic, A. V. Penacchioni, G. B. Pisani, J. A. Rueda, and Y. Wang. On binary-driven hypernovae and their nested late X-ray emission. $A \& A, 565: \mathrm{L} 10$, May 2014. doi:10.1051/0004-6361/201423812

T. Sakamoto, J. E. Hill, R. Yamazaki, L. Angelini, H. A. Krimm, G. Sato, S. Swindell, K. Takami, and J. P. Osborne. Evidence of Exponential Decay Emission in the Swift Gamma-Ray Bursts. ApJ, 669:1115-1129, November 2007. doi: $10.1086 / 521640$ 
T. Sakamoto, S. D. Barthelmy, L. Barbier, J. R. Cummings, E. E. Fenimore, N. Gehrels, D. Hullinger, H. A. Krimm, C. B. Markwardt, D. M. Palmer, A. M. Parsons, G. Sato, M. Stamatikos, J. Tueller, T. N. Ukwatta, and B. Zhang. The First Swift BAT Gamma-Ray Burst Catalog. ApJS, 175: 179-190, March 2008. doi:10.1086/523646

R. Sari and P. Mészáros. Impulsive and Varying Injection in Gamma-Ray Burst Afterglows. ApJL, 535:L33-L37, May 2000. doi:10.1086/312689

R. Sari, T. Piran, and R. Narayan. Spectra and Light Curves of Gamma-Ray Burst Afterglows. ApJL, 497:L17-L20, April 1998. doi:10.1086/311269

P. Schady, K. O. Mason, M. J. Page, M. de Pasquale, D. C. Morris, P. Romano, P. W. A. Roming, S. Immler, and D. E. vanden Berk. Dust and gas in the local environments of gamma-ray bursts. MNRAS, 377:273-284, May 2007. doi:10.1111/j.1365-2966.2007.11592.x

B. E. Schaefer. The Hubble Diagram to Redshift $>6$ from 69 Gamma-Ray Bursts. ApJ, 660:16-46, May 2007. doi:10.1086/511742

D. Stern, R. Jimenez, L. Verde, S. A. Stanford, and M. Kamionkowski. Cosmic Chronometers: Constraining the Equation of State of Dark Energy. II. A Spectroscopic Catalog of Red Galaxies in Galaxy Clusters. ApJS, 188:280-289, May 2010. doi:10.1088/0067-0049/188/1/280

J. Sultana, D. Kazanas, and K. Fukumura. Luminosity Correlations for Gamma-Ray Bursts and Implications for Their Prompt and Afterglow Emission Mechanisms. ApJ, 758: 32, October 2012. doi:10.1088/0004-637X/758/1/32

J. Sultana, D. Kazanas, and A. Mastichiadis. The Supercritical Pile Gamma-Ray Burst Model: The GRB Afterglow Steep Decline and Plateau Phase. ApJ, 779:16, December 2013. doi:10.1088/0004-637X/779/1/16

N. Suzuki, D. Rubin, C. Lidman, G. Aldering, R. Amanullah, K. Barbary, L. F. Barrientos, J. Botyanszki, M. Brodwin, N. Connolly, K. S. Dawson, A. Dey, M. Doi, M. Donahue, S. Deustua, P. Eisenhardt, E. Ellingson, L. Faccioli, V. Fadeyev, H. K. Fakhouri, A. S. Fruchter, D. G. Gilbank, M. D. Gladders, G. Goldhaber, A. H. Gonzalez, A. Goobar, A. Gude, T. Hattori, H. Hoekstra, E. Hsiao, X. Huang, Y. Ihara, M. J. Jee, D. Johnston, N. Kashikawa, B. Koester, K. Konishi, M. Kowalski, E. V. Linder, L. Lubin,

J. Melbourne, J. Meyers, T. Morokuma, F. Munshi, C. Mullis, T. Oda, N. Panagia, S. Perlmutter, M. Postman, T. Pritchard, J. Rhodes, P. Ripoche, P. Rosati, D. J. Schlegel, A. Spadafora, S. A. Stanford, V. Stanishev, D. Stern, M. Strovink, N. Takanashi, K. Tokita, M. Wagner, L. Wang, N. Yasuda, H. K. C. Yee, and T. Supernova Cosmology Project. The Hubble Space Telescope Cluster Supernova Survey. V. Improving the Dark-energy Constraints above z $i, 1$ and Building an Early-type-hosted Supernova Sample. ApJ, 746: 85, February 2012. doi:10.1088/0004-637X/746/1/85

E. Troja, G. Cusumano, P. T. O'Brien, B. Zhang, B. Sbarufatti, V. Mangano, R. Willingale, G. Chincarini, J. P. Osborne, F. E. Marshall, D. N. Burrows, S. Campana, N. Gehrels, C. Guidorzi, H. A. Krimm, V. La Parola, E. W. Liang, T. Mineo, A. Moretti, K. L. Page, P. Romano, G. Tagliaferri, B. B. Zhang, M. J. Page, and P. Schady. Swift Observations of GRB 070110: An Extraordinary X-Ray Afterglow Powered by the Central Engine. ApJ, 665:599-607, August 2007. doi:10.1086/519450

R. Tsutsui, D. Yonetoku, T. Nakamura, K. Takahashi, and Y. Morihara. Possible existence of the $\mathrm{E}_{p}-\mathrm{L}_{p}$ and $\mathrm{E}_{p}-\mathrm{E}_{\text {iso }}$ correlations for short gamma-ray bursts with a factor 5-100 dimmer than those for long gamma-ray bursts. MNRAS, 431: 1398-1404, May 2013. doi:10.1093/mnras/stt262
Y. Urata, R. Yamazaki, T. Sakamoto, K. Huang, W. Zheng, G. Sato, T. Aoki, J. Deng, K. Ioka, W. Ip, K. S. Kawabata, Y. Lee, X. Liping, H. Mito, T. Miyata, Y. Nakada, T. Ohsugi, Y. Qiu, T. Soyano, K. Tarusawa, M. Tashiro, M. Uemura, J. Wei, and T. Yamashita. Testing the External-Shock Model of Gamma-Ray Bursts Using the Late-Time Simultaneous Optical and X-Ray Afterglows. ApJL, 668:L95-L98, October 2007. doi:10.1086/522930

V. V. Usov. Millisecond pulsars with extremely strong magnetic fields as a cosmological source of gamma-ray bursts. Nature, 357:472-474, June 1992. doi:10.1038/357472a0

H. van Eerten. Self-similar relativistic blast waves with energy injection. MNRAS, 442:3495-3510, August 2014a. doi:10.1093/mnras/stu1025

H. J. van Eerten. Gamma-ray burst afterglow plateau break time-luminosity correlations favour thick shell models over thin shell models. MNRAS, 445:2414-2423, December 2014b. doi:10.1093/mnras/stu1921

F.-Y. Wang, S. Qi, and Z.-G. Dai. The updated luminosity correlations of gamma-ray bursts and cosmological implications. MNRAS, 415:3423-3433, August 2011. doi:10.1111/j.1365-2966.2011.18961.x

F. Y. Wang, Z. G. Dai, and E. W. Liang. Gamma-ray burst cosmology. New Astronomy Reviews, 67:1-17, August 2015. doi:10.1016/j.newar.2015.03.001

H. Wei and S.-N. Zhang. Reconstructing the cosmic expansion history up to redshift $\mathrm{z}=6.29$ with the calibrated gamma-ray bursts. European Physical Journal C, 63:139-147, September 2009. doi:10.1140/epjc/s10052-009-1086-z

D. H. Weinberg, M. J. Mortonson, D. J. Eisenstein, C. Hirata, A. G. Riess, and E. Rozo. Observational probes of cosmic acceleration. PhR, 530:87-255, September 2013. doi:10.1016/j.physrep.2013.05.001

R. A. M. J. Wijers, M. J. Rees, and P. Meszaros. Shocked by GRB 970228: the afterglow of a cosmological fireball. MNRAS, 288:L51-L56, July 1997.

R. Willingale, P. T. O'Brien, J. P. Osborne, O. Godet, K. L. Page, M. R. Goad, D. N. Burrows, B. Zhang, E. Rol, N. Gehrels, and G. Chincarini. Testing the Standard Fireball Model of Gamma-Ray Bursts Using Late X-Ray Afterglows Measured by Swift. ApJ, 662:1093-1110, June 2007. doi:10.1086/517989

R. Willingale, F. Genet, J. Granot, and P. T. O'Brien. The spectral-temporal properties of the prompt pulses and rapid decay phase of gamma-ray bursts. MNRAS, 403:1296-1316, April 2010. doi:10.1111/j.1365-2966.2009.16187.x

R. Yamazaki. Prior Emission Model for X-ray Plateau Phase of Gamma-Ray Burst Afterglows. ApJL, 690:L118-L121, January 2009. doi:10.1088/0004-637X/690/2/L118

S. X. Yi, Z. G. Dai, X. F. Wu, and F. Y. Wang. X-Ray Afterglow Plateaus of Long Gamma-Ray Bursts: Further Evidence for Millisecond Magnetars. ArXiv e-prints, January 2014.

D. Yonetoku, T. Murakami, T. Nakamura, R. Yamazaki, A. K. Inoue, and K. Ioka. Gamma-Ray Burst Formation Rate Inferred from the Spectral Peak Energy-Peak Luminosity Relation. ApJ, 609:935-951, July 2004. doi:10.1086/421285

E. Zaninoni, M. G. Bernardini, R. Margutti, S. Oates, and G. Chincarini. Gamma-ray burst optical light-curve zoo: comparison with X-ray observations. $A \mathscr{E} A, 557: \mathrm{A} 12$, September 2013. doi:10.1051/0004-6361/201321221

B. Zhang and S. Kobayashi. Gamma-Ray Burst Early Afterglows: Reverse Shock Emission from an Arbitrarily Magnetized Ejecta. ApJ, 628:315-334, July 2005. doi:10.1086/429787 
B. Zhang and P. Mészáros. Gamma-Ray Burst Afterglow with

Continuous Energy Injection: Signature of a Highly

Magnetized Millisecond Pulsar. ApJL, 552:L35-L38, May

2001. doi:10.1086/320255

B. Zhang and H. Yan. The Internal-collision-induced Magnetic

Reconnection and Turbulence (ICMART) Model of

Gamma-ray Bursts. ApJ, 726:90, January 2011.

doi:10.1088/0004-637X/726/2/90
B. Zhang, E. Liang, K. L. Page, D. Grupe, B.-B. Zhang, S. D. Barthelmy, D. N. Burrows, S. Campana, G. Chincarini, N. Gehrels, S. Kobayashi, P. Mészáros, A. Moretti, J. A. Nousek, P. T. O'Brien, J. P. Osborne, P. W. A. Roming, T. Sakamoto, P. Schady, and R. Willingale. GRB Radiative Efficiencies Derived from the Swift Data: GRBs versus XRFs, Long versus Short. ApJ, 655:989-1001, February 2007a. doi: $10.1086 / 510110$

B.-B. Zhang, E.-W. Liang, and B. Zhang. A Comprehensive Analysis of Swift XRT Data. I. Apparent Spectral Evolution of Gamma-Ray Burst X-Ray Tails. ApJ, 666:1002-1011, September 2007b. doi:10.1086/519548 